\title{
CHEMICAL MODIFICATIONS OF THE SUBTILISINS WITH SPECIAL REFERENCE TO THE BINDING OF LARGE SUBSTRATES. A REVIEW.
}

\author{
by \\ IB SVENDSEN \\ Department of Chemistry - Carisberg Laboratory \\ Gamle Carlsberg Vej 10 - DK-2500 Copenhagen, Valby
}

Key words: Subtilisin, Chemical modifications, Secondary binding sites

\begin{abstract}
Subtilisin type Carlsberg and subtilisin BPN' are two well characterized extracellular proteases from Bacillus subtilis and Bacillus amyloquefaciens, respectively. The present review summarizes the various chemical modification studies which have been performed with these enzymes. Of special interest has been those modifications which lead to changes in the enzymatic properties with regard to the hydrolysis of polypeptide substrates but not small synthetic ester substrates. In this way it is possible to obtain information about how large an area of the surface of the enzyme is involved in the binding of natural substrates (e.g. clupein, gelatine, casein). Nitration, iodination, glutarylation or succinylation of the subtilisins leads to increases in the hydrolyses of clupein and gelatine, while modification of carboxyl groups leads to a decrease. In all these cases the hydrolysis of small ester substrates is almost unaffected. A section of the review deals with the comparison between the results obtained by chemical modification studies and the two other methods available for the study of "secondary binding sites": $\mathrm{X}$-ray diffraction analyses and kinetic studies with synthetic polypeptides. The productive binding mode for polypeptides proposed by KRAUT and coworkers is in agreement with the results obtained by nitration of the subtilisins. However, results from our laboratory and the literature point towards more than one mode of productive binding. These results are discussed. Finally a comparison is made between the secondary binding sites in subtilisin and other proteolytic enzymes, especially the serine proteases. The importance of secondary interactions between enzyme and substrate is discussed.
\end{abstract}




\section{CONTENTS}

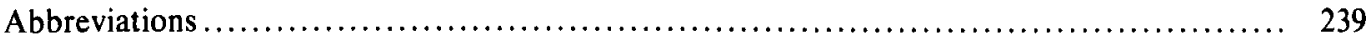

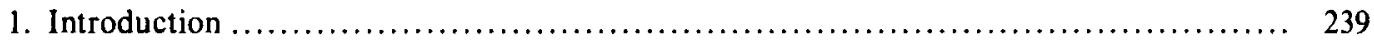

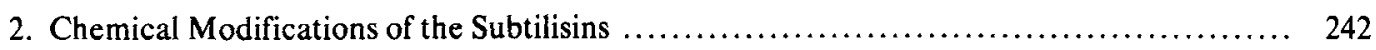

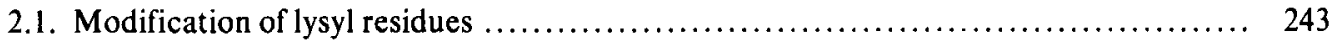

2.1.1. Carbamylation .............................................. 243

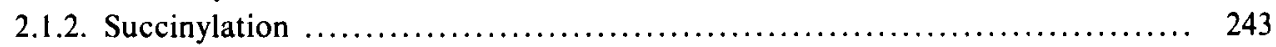

2.1.3. Glutarylation ................................................... 243

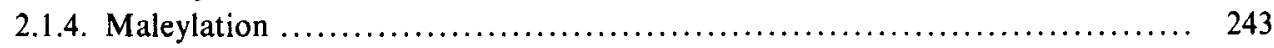

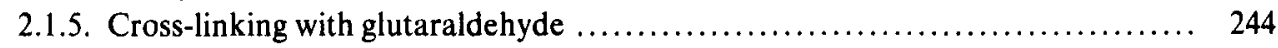

2.2. Modification of tyrosyl residues ...................................... 244

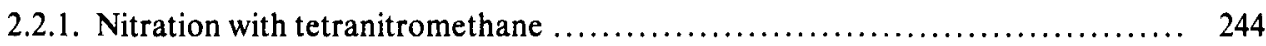

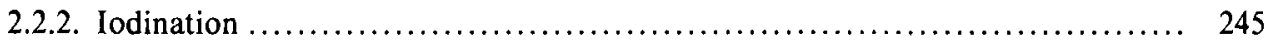

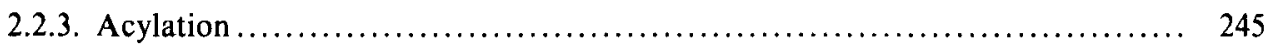

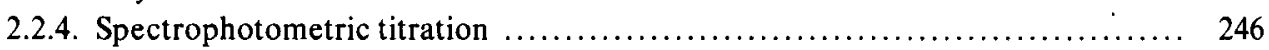

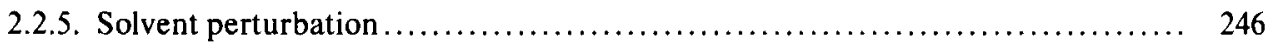

2.2.6. Circular polarization of fluorescence ................................. 246

2.2.7. Comparison with the model ....................................... 246

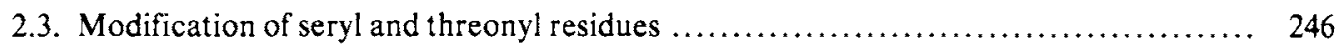

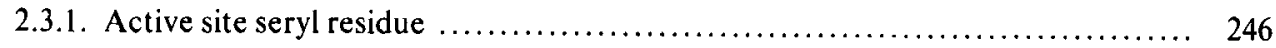

2.3.2. Other seryl and threonyl residues ................................. 249

2.4. Modification of histidyl residues ...................................... 249

2.5. Modification of methionyl residues ...................................... 249

2.6. Modification of tryptophanyl residues .................................... 250

2.7. Modification of glutamic/aspartic acid residues ............................ 251

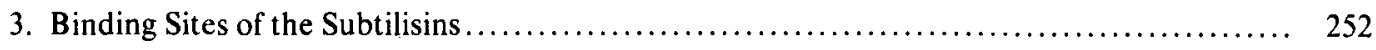

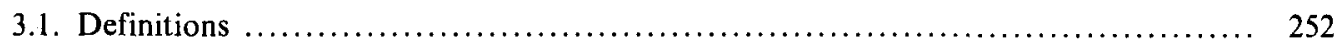

3.2. Mechanism of action of the subtilisins ................................ 253

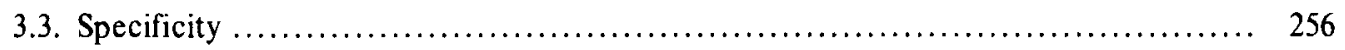

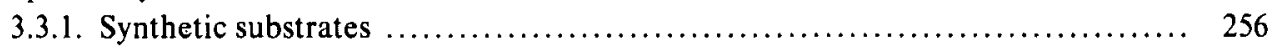

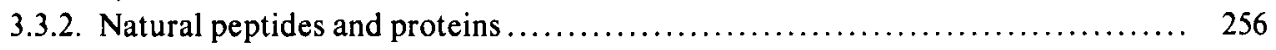

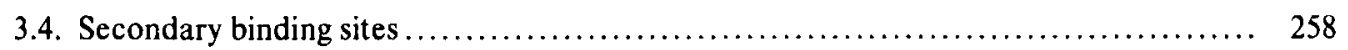

3.4.1. Kinetic studies ............................................... 258

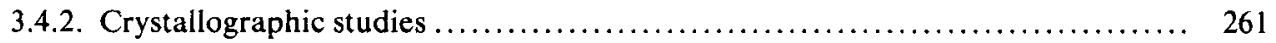

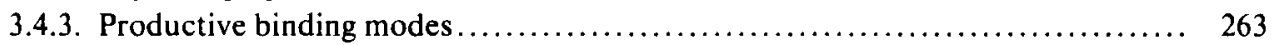

3.4.4. Chemical modifications ............................................ 264

3.4.5. Effect of neutral salts ......................................... 270

3.4.6. Effect of alcohols and guanidinium hydrochloride $\ldots \ldots \ldots \ldots \ldots \ldots \ldots \ldots \ldots, 272$ 


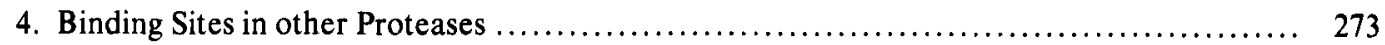

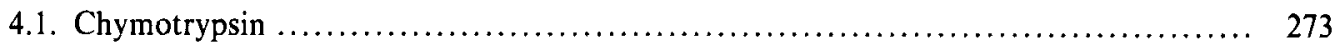

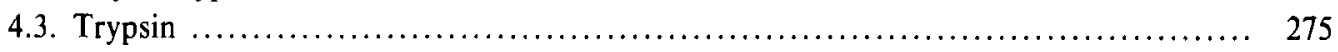

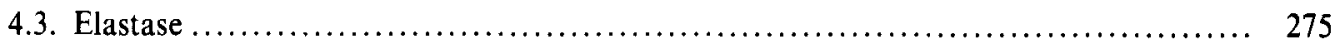

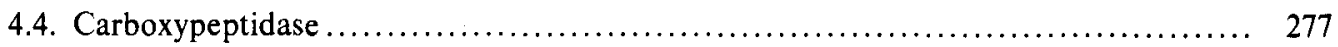

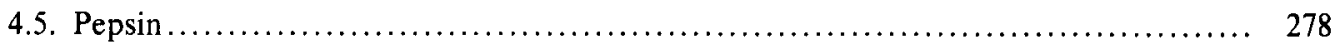

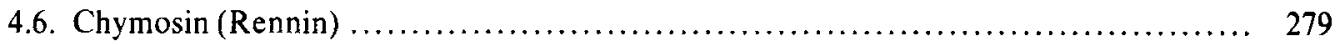

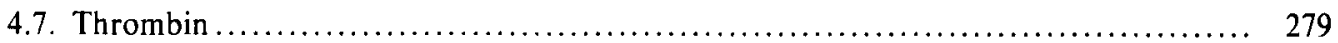

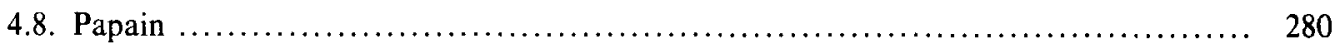

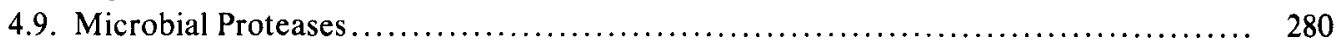

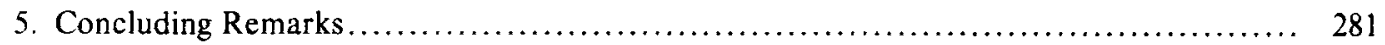

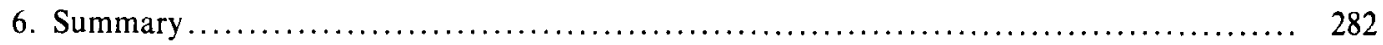

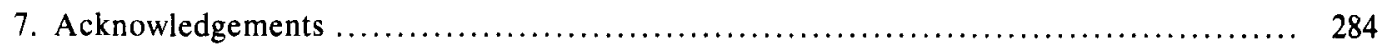

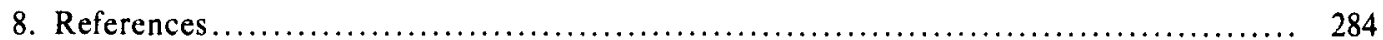

His, Cys, etc: Three-letter code for amino acids according to the recommendations of the CBN-Commission (see f.ex. J.Biol.Chem. 247.977 (1972)

$\mathrm{Tyr}_{10} \quad$ : refers to the position of the amino acid in question in the protein sequence

ATEE : N-acetyl-i-tyrosine ethyl ester

BAEE : N-benzoyl-L-arginine methyl ester

Boc : t-butyloxycarbinol

BTEE : N-benzoyl-L-tyrosine ethyl ester

$\mathrm{Bz}$ : benzoyl

CMC : N-cyclohexyl-N' $\beta$-(N-methyl morpholino)ethyl-carbodiimide

DFP : diisopropylfluorophosphate

DIP : diisopropylphosphoryl

DNA : deoxyribonucleic acid

DPCC : diphenylcarbonylchloride

EDC : N-ethyl-N'-1,3-dimethylaminopropyl carbodiimide

NBS : N-bromosuccinimide

$\mathrm{NBz} \quad$ : p-nitrobenzyl

NCDC : 2-nitro-4-carboxyphenyl-N,N-diphenyl carbamate

$\mathrm{NO}_{2}$ Tyr : 3-nitrotyrosine

OMe : methyl ester

PMS : phenyl methyl sulfonyl

PMSF : phenyl methyl sulfonylfluoride

PTI : pancreatic trypsin inhibitor

TAME : N-tosyl-L-arginine methyl ester

TLCK : N-tosyl-L-lysine chloromethyl ketone

TNBS : 2,4,6-trinitrobenzenesulfonic acid

TNM : tetranitromethane

TPCK : N-tosyl-L-phenylalanine chloromethyl ketone

\section{INTRODUCTION}

The subtilisins (EN 3.4.21.14) are a group of extracellular, alkaline proteases produced by various species of Bacillus. One of the first proteolytic enzymes of bacterial origin to be isolated was an enzyme from Bacillus subtilis which was able to transform ovalbumin into plakalbumin $(59,104,105)$.

This enzyme, now called subtilisin type Carlsberg, has since its isolation been studied at the Carlsberg Laboratory together with the closely related subtilisin type Novo which is identical to subtilisin BPN' (or "Nagarse") originally isolated in Japan by HAGIHARA et al. (62). The identity of the two proteases was indicated from enzymatic studies (80) as well as amino acid composition (83) and conclusively demonstrated by sequence studies (136) and Xray crystallography $(41,216)$. In the present review subtilisin type Novo and subtilisin BPN' are treated as one enzyme, and will be referred to as subtilisin BPN'. It was originally believed that both subtilisin type Carlsberg and subtilisin BPN' were produced by different strains of Bacillus subtilis, but it was later shown that subtilisin BPN' probably originates from another species: Bacillus amyloliquefaciens (212). An increasing number of alkaline proteases from different species of Bacillus have been 
characterized $(24,61,92.93,100,120)$ and it appears that they resemble either subtilisin type Carlsberg or subtilisin BPN' in their amino acid composition and catalytic behaviour (93). Only the two best characterized enzymes, subtilisin type Carlsberg and subtilisin BPN' will be discussed and only to the extent that the main object of the review will be fully intelligible. A broader discussion of the subtilisins is given in recent reviews $(113,142)$.

The amino acid sequences of both subtilisin type Carlsberg and subtilisin BPN' were deter- mined by E. L. SMITH and coworkers (109, 182). Both enzymes consist of a single polypeptide chain and contain 275 residues (BPN') and 274 residues (Carlsberg), respectively. The amino acid sequences are shown in Fig. 1. The homology between the enzymes is evident, although there are deviations in 85 positions, including the single deletion. Since cystine (or cysteine) is not present in these enzymes, disulphide bridges are of no importance for the tertiary structure. None the less, the subtilisins appear to have a very rigid structure, being

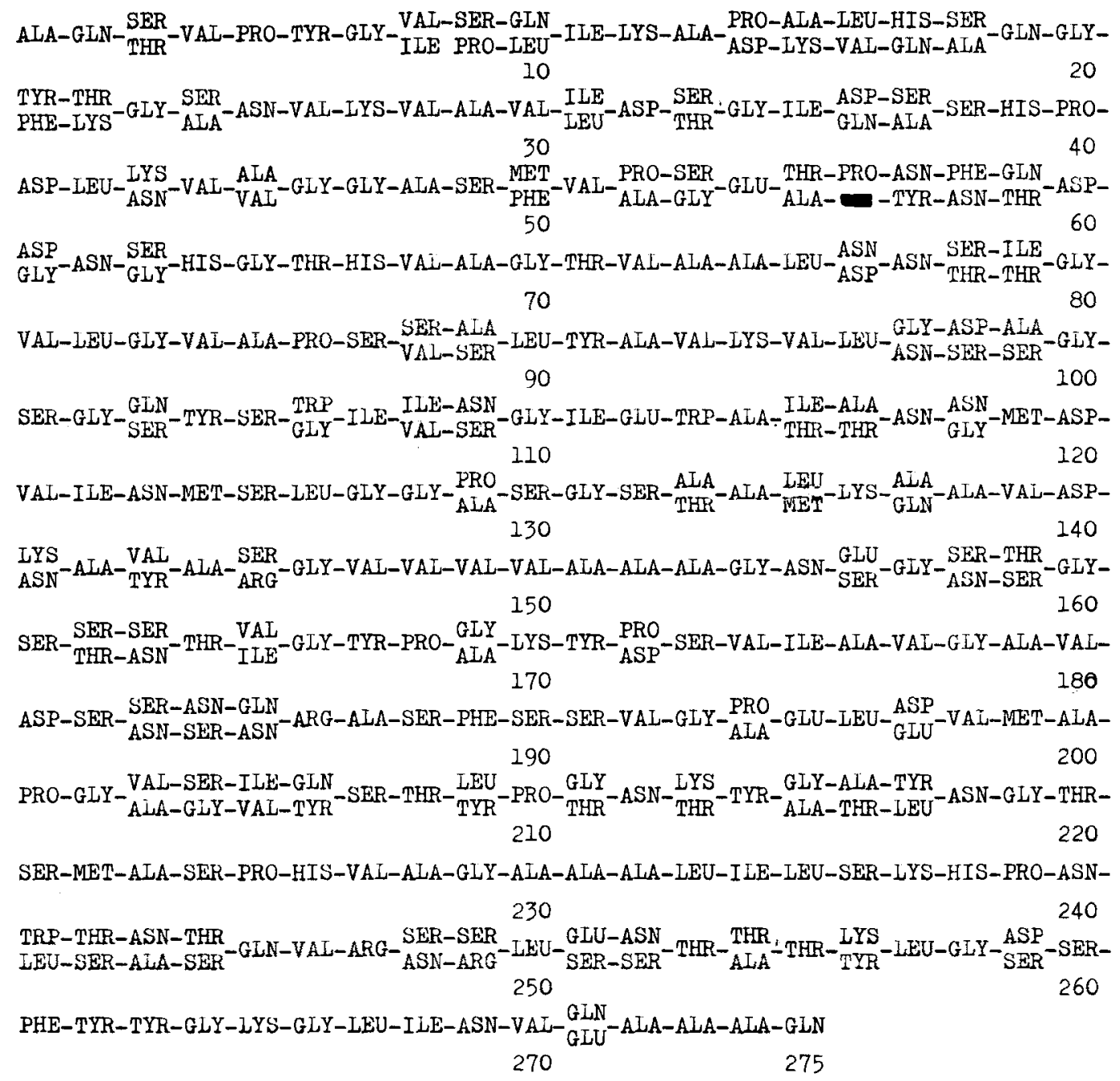

Figure 1. The amino acid sequences of subtilisin $\mathrm{BPN}^{\prime}$ and subtilisin type Carlsberg. In those cases where the two enzymes differ in sequence the upper line refers to subtilisin BPN' and the lower line to subtilisin type Carlsberg. The deletion in position 56 is indicated by a 
stable in hydrogen-bond breaking media (e.g. 8 M-urea) or media that weaken hydrophobic interactions (e.g. 50\% alcohol) (141). The reason for this remarkable stability is probably that each of the two types of forces mentioned above are strong enough to keep the molecule together by itself, so that weakening of only one type will have no denaturing effect on the molecule. As mentioned above, crystallographic studies of subtilisin BPN' (216) and subtilisin type Novo (41) have shown these enzymes to be identical. Subtle differences, observed with respect to the binding of anions and water molecules, may be due to the different ways in which the crystals were produced in the two cases.

While the three-dimensional structure of subtilisin $\mathrm{BPN}^{\prime}$ is known it has been difficult to produce crystals of subtilisin type Carlsberg suitable for X-ray diffraction studies. TSERNOGLOU and PETSKo (203) have recently obtained preliminary data on this enzyme measured to $3.4 \AA$ resolution. Therefore, an exact comparison of the two structures seems possible within the near future. However, it was pointed out by WRIGHT et al. (216) that all but one of the differences between the amino acid sequences of the two enzymes occur on the surface of subtilisin BPN' while the amino acid sequence comprising the interior of subtilisin BPN' $^{\prime}$ is nearly identical to the corresponding sequence of subtilisin type Carlsberg. Furthermore, the single difference in the interior was a conservative substitution of an isoleucine in subtilisin BPN' by a leucine in subtilisin type Carlsberg. On the basis of these observations, a three-dimensional model of the Carlsberg enzyme, based on the coordinates available for subtilisin BPN' $^{\prime}$ and the assumption that the folding of the two enzymes were identical, was built at the Carlsberg Laboratory. No difficulties were encountered in constructing the model. The single deletion in position 56 occurs in a turn of the chain, and residues 55 and 57 were connected through a peptide bond without difficulty. With the two models at hand (the BPN' model was also constructed), it was possible to study the topography of the enzyme surfaces and to make comparisons between the two enzymes.
Such comparisons were especially valuable for the approach chosen by the present author in the study of the subtilisins. Chemical modification studies have been performed with all of the functional amino acid side-chains except the guanidinium group of arginine, and section 2 reviews the chemical modification studies of the subtilisins performed at the Carlsberg Laboratory as well as by other investigators.

Some amino acid side-chains appear to be without importance for the function of the enzymes, while chemical modifications of others impair or totally block catalysis. However, it was found that in some cases the rate of hydrolysis of certain large substrates is enhanced while that of small substrates is unaffected $(85,88,188)$. Chemical modifications of proteases therefore appear to be one way of studying the binding of large substrates.

Studies of the mechanism of action of proteolytic enzymes have to a very large extent been performed with small substrates. Since most of the proteases also function as esterases it has been common to use esters of amino acids as substrates in this kind of work, and much ingenuity has been shown in synthesizing substrates with especially labile ester bonds or containing chromophoric groups that facilitate the kinetic measurements. Proteolytic enzymes, however, are produced by the organisms with the purpose of hydrolyzing proteins and peptides. Therefore attention must be focused on these substrates as well, in order to obtain a more complete understanding of the catalytic behavior of the proteases. The complexity of their action on proteins was already anticipated by EMIL FISCHER who in 1903 wrote (45):

"Aus den vorstehenden Beobachtungen geht hervor, dass die Hydrolyse von Dipeptiden und ihren Derivaten durch Pancreatin von sehr verschiedenen Factoren, d.h. von der Natur der Aminosäure, von ihrem sterischen Bau und endlich bei den Derivaten auch von der Zusammesetzung des ganzen Moleküls abhängig ist. Dieses Unterschiede werden sich bei den Höheren Polypeptiden zweifellos in grösserer Mannigfaltigkeit wiederholen und man kann sich darnach ungefär eine Vorstellung darüber 
machen, wie verschiedenartig der Angriff des Enzyme sich bei den noch viel complizirter zusammengesetzten Proteinst offen gestalten muss".

Some of the questions which have to be answered when discussing the binding of proteins to proteolytic enzymes are: How large an area of the enzyme surface is involved in the binding, and in which way does the binding at multiple points influence the rate of hydrolysis and the specificity of the enzyme? Are all large substrates bound in an identical manner, or are there different binding modes for different substrates? Are different binding modes for the same substrate a possibility?

Different approaches to the study of binding of large polypeptides to proteolytic enzymes have been used. These include:

1. Kinetic studies involving chemically modified enzymes.

2. Kinetic studies using synthetic polypeptides.

3. Crystallographic studies of enzyme-inhibitor complexes.

Although many investigations involving chemical modifications of proteases have been published, only few are discussed in terms of binding of large substrates. The main interest has usually been concentrated around the participation of functional side-chains in the catalytic process or in maintaining the tertiary structure of the enzyme. The enzymatic consequences of the chemical modifications of subtilisin $(3,88,188)$ which have been shown to influence specifically the binding of large substrates are discussed (section 3.4.4) in relation to those results obtained by other laboratories using methods 2 and 3.

Kinetic studies involving synthetic polypeptides have been performed with the subtilisins (sections 3.4.1 and 3.4.3) and a number of other proteases (section 4). In most cases peptides have been used in which side-chains, stereochemical properties, and the length of the peptide-chain have been varied. Kinetic studies of natural polypeptides of known sequence, as exemplified by the extensive use of the B-chain of oxidized insulin (85), may also give useful information about the specificity.

Chemical modifications of the side-chains in natural polypeptides seem to offer another possibility for studying the binding of large substrates to proteases, but no systematic studies of such an approach have been published. The Bchain of insulin appears to be very suitable for this purpose since it contains several amino acid side-chains (His, Cys, Glu, Tyr, Arg, Ser, Thr and Lys) which can be modified in various ways.

Detailed crystallographic studies concerning the binding of synthetic polypeptide inhibitors to subtilisin BPN $^{\prime}(159,160)$ as well as to chymotrypsin, trypsin and elastase $(175,179$, 196) have been performed. These results are compared in section 4. Binding of natural polypeptide inhibitors to proteolytic enzymes is of special interest, and results based on model building have been published for complexes of pancreatic trypsin inhibitor with trypsin and chymotrypsin (75). A recent preliminary study of the complex between subtilisin BPN ${ }^{*}$ and an inhibitor from Streptomyces has also been reported (168).

\section{CHEMICAL MODIFICATIONS OF THE SUBTILISINS}

In this section the chemical modification

Table I

The amino acid composition of subtilisin type Carlsberg and subtilisin BPN'.

\begin{tabular}{l|r|r}
\hline Amino acid & Carlsberg & BPN \\
\hline Lysine & 9 & 11 \\
Histidine & 5 & 6 \\
Arginine & 4 & 2 \\
Aspartic acid & 9 & 11 \\
Asparagine & 19 & 17 \\
Threonine & 19 & 13 \\
Serine & 32 & 37 \\
Glutamic acid & 5 & 4 \\
Glutamine & 7 & 11 \\
Proline & 9 & 14 \\
Glycine & 35 & 33 \\
Alanine & 41 & 37 \\
Valine & 31 & 30 \\
Methionine & 5 & 5 \\
Isoleucine & 10 & 13 \\
Leucine & 16 & 15 \\
Tyrosine & 13 & 10 \\
Phenylalanine & 4 & 3 \\
Tryptophan & 1 & 3 \\
Cysteine, cystine & 0 & 0 \\
\hline
\end{tabular}


studies performed with the functional sidechains of the subtilisins will be reviewed. The number and kind of functional side-chains in the two enzymes are given in Table I together with the total amino acid composition. In those cases where other methods have been used (for example potentiometric titration or spectrophotometric studies), these are also included. However, the discussion of those cases where the modifications led to changes in the binding properties of the enzymes with respect to large substrates will be postponed to section 3 , where it is included in a general discussion of the binding properties of the subtilisins.

\subsection{Modification of Lysyl Residues}

Among the large number of reagents available for modification of lysyl residues $(32,208)$ the four acylating agents potassium cyanate, succinic anhydride, glutaric anhydride, and maleic anhydride have been used in the studies of the subtilisins.

\subsubsection{Carbamylation}

Of the 11 lysyl side-chains in subtilisin BPN', 10.3 were found to react with potassium cyanate to form homocitrulline groups (187). The large change in net charge that takes place by replacing 10 to 11 positively charged lysines with neutral homocitrullines did not influence the tertiary structure of the enzyme to any major extent as shown by the unchanged optical rotatory dispersion parameters, $b_{o}$ and $\lambda_{c}$, and sedimentation velocity, $\mathrm{s}_{20, \mathrm{w}}^{0}$. The susceptibility of modified enzyme to acid denaturation was identical to that of the unmodified one, showing that a simple change of net charge cannot explain the unfolding in acid medium (141).

In addition to the $\varepsilon$-amino groups of lysine, the $\mathrm{N}$-terminal $\alpha$-amino group was also modified. This was shown by carrying out an end group determination according to the method of STARK (191). These findings are in agreement with the proposed tertiary structure (216). All of the lysyl side-chains but one are freely accessible on the surface of the molecule. The side-chain of $\mathrm{Lys}_{94}$ is located in a surface crevice and probably reacts much more slowly with potassium cyanate than the rest of the lysyl residues. Inactivation took place concomitantly with the modification of the $\alpha$ - and $\varepsilon$-amino groups. However, it was shown that modification of other groups must be responsible for the loss of activity, since reactivation was easily

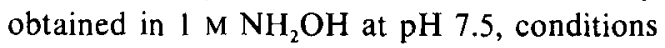
which do not affect the modified $\varepsilon$ - and $a$ amino groups. Furthermore the rate of inactivation was strongly $\mathrm{pH}$-dependent being much faster at $\mathrm{pH} 6$ than 8 , while the rate of reaction with the amino groups is $\mathrm{pH}$-independent in the range 6 to 9 (192). Since the activity of reactivated subtilisin $\mathrm{BPN}^{\prime}$ was identical to that of the unmodified enzyme towards $0.5 \%$ clupein it was concluded that the lysyl side chains, as well as the $\mathrm{N}$-terminal amino group, were of no importance for the activity of this enzyme.

\subsubsection{Succinylation}

GOUNARIS and OTTESEN (56) have shown that 10 of the 11 lysyl residues in subtilisin BPN' are susceptible to reaction with succinic anhydride. Among the other side-chains which could potentially be modified, tyrosyl residues were shown to be intact. No analyses were performed, however, for possible reactions with histidyl, seryl, or threonyl residues.

In subtilisin type Carlsberg, 6 to 7 of the 9 lysyl residues can be succinylated (88). Although the reaction between subtilisin type Carlsberg and succinic anhydride led to remarkable changes in the enzymatic properties of the enzyme (see section 3.4.4), it was shown that these were not related to modification of lysyl side-chains.

\subsubsection{Glutarylation}

JOHANSEN (88) also modified subtilisin type Carlsberg with glutaric anhydride and showed that 8 of the 9 lysyl residues were modified. The concomitant changes in enzymatic properties (see section 3.4.4) were also in this case unrelated to the modification of lysyl residues. As in subtilisin BPN', all lysyl side-chains but one are freely exposed on the enzyme surface (as judged from the tentative three-dimensional model), the exception again being Lys $_{94}$.

\subsubsection{Maleylation}

Maleylation of subtilisin BPN' has been $^{\prime}$ performed with maleic anhydride and 2,3- 
dimethyl maleic anhydride (48) and 10.7 of the 11 lysyl residues were modified in this case. The enzymatic consequences of this modification will be discussed in relation to the hydrolysis of salmine sulphate (see section 3.4.4).

\subsubsection{Cross-linking with Glutaraldehyde}

Although glutaraldehyde is used as a crosslinking reagent $(60,150)$ rather than as a probe for the role of lysyl residues in the structurefunction relationship of proteins, the reaction with this reagent is included in this section since it is believed that cross-linking takes place via lysyl residues. The reaction mechanism, however, is not yet known. OGATA et al. (132) used glutaraldehyde to cross-link subtilisin BPN'. The insoluble derivative obtained was still enzymatically active although the rate of hydrolysis of various substrates was greatly reduced relative to that of the unmodified enzyme.

\subsection{Modification of Tyrosyl Residues}

The phenolic side chain of tyrosyl residues is an excellent object for chemical modification since it can be substituted both in the ring (nitration and iodination) and at the phenolic hydroxyl group (acylation). The spectral characteristics of tyrosyl side-chains make them suitable for spectroscopic studies such as spectrophotometric titration and solvent perturbation. The tyrosyl residues of the subtilisins have been studied by means of all the methods mentioned above and are therefore the best characterized functional groups in these enzymes.

\subsubsection{Nitration with Tetranitromethane}

The introduction of tetranitromethane (TNM) by VALLEE and coworkers (184) as a nitrating agent for proteins appeared at first very promising in terms of selectivity and mild reaction conditions. TNM was thought only to react with tyrosyl side-chains by converting them into 3-nitrotyrosyl residues, and to react with the sulphydryl group of cysteinyl residues in an oxidation reaction leading to the formation of disulphide bonds or sulphinic acid. It was later shown that reaction with tryptophanyl, methionyl and histidyl residues can also take place $(185,186)$ at $\mathrm{pH}$ 's higher than 8 or at large molar excesses of TNM. While these side reactions can be minimized or eliminated, reactions leading to polymerization products pose a more serious problem. According to BRUICE et al. (28) the nitration of tyrosine proceeds via a free radical mechanism. Therefore the reaction of the phenoxy radicals with one another may give rise to coupling products. If the reaction takes place between radicals from different molecules polymerization occurs. This is demonstrated in several cases (for example 20, 107, 209).

WILliams and Lowe (213) have shown that treatment of tyrosine at $\mathrm{pH} 9.0$ led to formation of di-tyrosine, tri-tyrosine, and higher homologues. They also isolated di- and tri-tyrosine from acid hydrolysates of insulin and ovotransferrin, provided that the ratio of TNM to tyrosine was $1: 1$.

It was shown by SVENDSEN (188) that 9 out of 13 tyrosyl residues in subtilisin type Carlsberg and 8 out of 10 in subtilisin BPN $^{\prime}$ reacted with TNM. However, the sum of tyrosine and 3-nitrotyrosine determined by amino acid analysis never exceeded 11 in subtilisin type Carlsberg except at very low degree of substitution. This observation suggested that cross-linking had taken place. It was shown by gelfiltration on Sephadex G100 that polymerization took place to only a limited extent, and that the number of 3-nitrotyrosines and tyrosines were the same in the monomer and the polymer (which presumably was a dimer) (190). The observed side reaction probably leads to intramolecular crosslinks between tyrosyl residues situated close together on the surface of the molecule or to cross-links between tyrosyl residues in the molecule and tyrosyl residues in autolysis peptides which are always present in the reaction mixture. Although most of the tyrosines can be nitrated in both subtilisins they do not react with the same rate. Thus MARKLAND (111) has shown that five tyrosyl residues in sub-

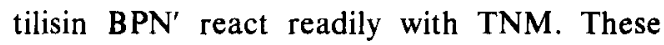
were identified as tyrosyl residues $6,21,104$, 217 , and 262. Tyrosyl residues 91, 263, and either/or $167 / 171$ were partially modified (183). In subtilisin type Carlsberg the following assignments were made (190): tyrosyl residues 
$57,104,143,206$, and 209 were fully nitrated, tyrosyl residue 91 was partially nitrated, tyrosyl residues 167 and 171 were either both nitrated partially or only one was fully nitrated. Residues 6 and 214 were not modified. No assignment could be given to residues 256,262 , and 263.

In both enzymes $\mathrm{Tyr}_{104}$ could be selectively modified $(183,190)$ but the reason for the high reactivity of this residue is still obscure.

\subsubsection{Iodination}

Iodination has been used for many decades as a means of modifying proteins. The reagent iodine in potassium iodide is not very specific since tryptophan, methionine, cysteine, histidine, as well as tyrosine can be modified. A systematic investigation by HUGHES and STRAESSLE (77) showed that the optimal reaction conditions for selective modification of

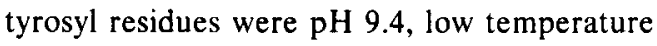
$\left(4^{\circ}\right)$, and addition of the reagent in small increments. These conditions were adopted by SVENDSEN (190) for the modification of tyrosyl residues in the subtilisins. As in the nitration experiments, 9 of the 13 residues in subtilisin type Carlsberg and 8 of the 10 residues in subtilisin BPN' could be modified. Amino acid analysis showed only tyrosyl residues to be modified. However, since tryptophan was not determined in these analyses, it cannot be excluded that this amino acid was modified as well. The decrease in enzymatic activity of subtilisin BPN' $^{\prime}$ observed when more than four tyrosyl residues were modified (190) may well be due to the undetected modification of tryptophanyl residues. $\mathrm{Tyr}_{104}$ is the first residue to be iodinated in subtilisin BPN'. This was shown by WEBER and KRAUT (211) who used ${ }^{125} \mathrm{ICl}$ as modifying reagent. The segment of the polypeptide chain containing labelled $\mathrm{Tyr}_{104}$ was isolated from a tryptic digest, and $\mathrm{X}$-ray analysis of the crystallized enzyme derivative also showed $\mathrm{Tyr}_{104}$ to be the only residue iodinated. In the crystalline state only four tyrosyl residues could be iodinated with an excess of iodine (216).Xray diffraction studies showed that 2 iodine atoms were located near $\mathrm{Tyr}_{104}$ (indicating a diodotyrosyl residue), 2 iodine atoms close to $\mathrm{Tyr}_{217}$ (disubstitution), one iodine between
$\mathrm{Tyr}_{167}$ and $\mathrm{Tyr}_{171}$ (monosubstitution of one of these residues), and one iodine atom close to $\mathrm{Tyr}_{21}$ (monosubstitution).

Both nitration and iodination led to pronounced changes in the enzymatic properties of the subtilisins. These changes will be discussed in detail in section 3.4.4.

\subsubsection{Acylation}

Acetylation of tyrosyl residues can be achieved by reaction with either $\mathrm{N}$-acetylimidazole or acetic anhydride (156). Of these reactions $\mathrm{N}$ acetylimidazole is the more selective since it preferentially reacts with tyrosyl residues while acetic anhydride reacts with $\alpha$ - and $\varepsilon$-amino groups with equal rate. Since an acetyl tyrosine is easily converted to tyrosine by treatment with hydroxylamine at alkaline $\mathrm{pH}$, the effect of acetylating lysyl residues alone can be studied. Using N-acetylimidazole, MYERS and Glazer (119) acetylated all tyrosyl residues in the phenyl methyl sulfonyl fluoride (PMSF) inhibited forms of both subtilisins. Inhibition of the enzymes prior to acetylation was found necessary because the active enzyme hydrolyzed the reagent much too fast for modification of tyrosyl residues to take place at the same time. Preliminary experiments by SvENDSEN showed that acetylation of the active subtilisins at $\mathrm{pH} 7.0-7.5$ and $3^{\circ} \mathrm{C}$ with an excess of acetic anhydride led to inactivation. Activity could be restored either by treatment with $1 \mathrm{M}$ hydroxylamine at $\mathrm{pH} 7.5$ or by raising the $\mathrm{pH}$ to 9.0. However, the reactivation was much slower with subtilisin Carlsberg than with subtilisin BPN'. Complete acetylation of the tyrosyl residues was not achieved in these experiments as estimated from the decrease in absorbance at $278 \mathrm{~nm}$. It is noteworthy that spontaneous deacylation of the acetylated intermediate is very fast when $\mathrm{N}$-acetylimidazole is used while an inactive derivative can be obtained with acetic anhydride as the acylating reagent.

Although succinyl tyrosyl residues are unstable due to deacylation by autocatalysis (155) the latter process is slow enough for the number of residues initially modified in the subtilisins to be determined. JOHANSEN (88) found that 9 residues could be modified in subtilisin type Carlsberg, and that deacylation took place in 
such a way that three of the modified residues deacylated with a rate one third of the rate of the other six. In subtilisin BPN' all ten residues could be modified (JOHANSEN, personal communication), and in this case a difference in the rate of deacylation was also observed.

In contrast to succinyltyrosyl residues the corresponding glutaryl derivatives were relatively stable in aqueous solution (88). 6.2 residues were modified in subtilisin type Carlsberg, one of which deacylated abnormally fast.

\subsubsection{Spectrophotometric Titration}

of the DFP-inhibited subtilisins (112) showed that some of the tyrosyl residues have abnormally high $\mathrm{pK}_{\mathrm{a}}$-values. Thus, in subtilisin type Carlsberg, 6 residues had $\mathrm{pK}_{\mathrm{a}}$ (app) of 9.92, 4 residues $\mathrm{pK}_{\mathrm{a}}(\mathrm{app})$ of 11.61 and 3 residues $\mathrm{pK}_{\mathrm{a}}$ above 12.5. In subtilisin $\mathrm{BPN}^{\prime} 5$ residues titrated with $\mathrm{pK}_{\mathrm{a}}(\mathrm{app})$ of $9.74,3$ residues with $\mathrm{pK}_{\mathrm{a}}(\mathrm{app})$ of 11.25 , and 2 residues had $\mathrm{pK}_{\mathrm{a}}$ above 12.5 .

\subsubsection{Solvent Perturbation}

Two papers have been published dealing with the solvent perturbation of aromatic side-chains in the subtilisins. MYERS and GLAZER (119) studied the effect of $20 \%$ ethylene glycol and concluded that 8.8 tyrosyl residues were exposed to solvent in both enzymes. This figure represented the summation of the effect on fully as well as partially exposed residues. Herskovits and Fuchs (69) used eight perturbants of increasing diameter in the study of subtilisin BPN'. They found $6.4 \pm 1$ as the apparent number of residues exposed. With $20 \%$ ethylene glycol the number was 6.0 . This figure differ markedly from that obtained by MYERS and GlAZER, but the solvent composition (apart from the $20 \%$ ethylene glycol) and $\mathrm{pH}$ was different in the two sets of experiments and may have influenced the accessibility of the tyrosyl residues.

\subsubsection{Circular Polarization of Fluorescence}

In a recent study by SCHLESSINGER et al. (172) using the technique of circular polarization of fluorescence, it was demonstrated that changes in the environment of some of the tyrosyl residues in subtilisin type Carlsberg take place in the $\mathrm{pH}$ range from 5.0 to 8.3 . In subtilisin BPN' $^{\prime}$ changes in the environment of both tyrosyl and tryptophanyl residues take place in the same $\mathrm{pH}$ interval. According to the authors the results indicate that local changes in conformation may be of importance for the activity of the subtilisins.

\subsubsection{Comparison with the Models}

Inspection of the three-dimensional model of subtilisin BPN' shows that no tyrosyl side-chain is buried deep in the interior of the molecule. On the contrary, all are located at or close to the surface. HeRskovits and FuCHS (69) have estimated the degree of exposure to range from 70 to $80 \%\left(\mathrm{Tyr}_{21}\right.$ and $\left.\mathrm{Tyr}_{262}\right)$ to 30 to $40 \%\left(\mathrm{Tyr}_{167}\right.$ and $\left.\mathrm{Tyr}_{214}\right)$. In subtilisin type Carlsberg all 13 residues are also located at or close to the surface of the molecule. However, the hydrophobic environments of some of the residues in both enzymes are so dominating that the $\mathrm{pK}_{\mathrm{a}}$ values are drastically increased. The possibility of hydrogen bonding of some of the residues exists and might also add to the increased $\mathrm{pK}_{\mathrm{a}}$ values.

Table II shows the number of tyrosyl residues that can be chemically modified by various reagents. There is a fair agreement between chemical reactivity and degree of exposure determined spectrophotometrically or by solvent perturbation. However, all residues can be modified by $\mathrm{N}$-acetylimidazole (119), a result which shows that the terms "buried" and nexposed" side-chains are not fixed values, but depend on the experimental conditions. Size, charge and solubility of a chemical reagent are important in determining the reaction with functional residues in proteins.

\subsection{Seryl and Threonyl Residues}

By definition the classification of the subtilisins as "serine proteases" (64) implies that the catalytic site contains a very reactive seryl residue, but chemical modification of other seryl and threonyl residues on the enzyme surface has also been reported.

\subsubsection{Active Site Seryl Residue}

In the original article on the purification of sub- 
Table II

Tyrosyl residues in subtilisin type Carlsberg and subtilisin BPN" available for various chemical modifications and for investigation by spectrochemical methods.

\begin{tabular}{l|c|c|c}
\hline \multicolumn{3}{c}{$\begin{array}{c}\text { Number of tyrosyl residues } \\
\text { in subtilisin }\end{array}$} \\
\hline Kind of experiment & Carlsberg & Novo & Ref. \\
\hline Amino acid hydrolysis & 13 & 10 & $(113)$ \\
Nitration (exhaustive) & 9 & 8 & $(188)$ \\
Nitration (mild) & 6 & 5 & $(188)$ \\
Nitration (mild) & - & 5 & $(183)$ \\
lodination (exhaustive) & 9 & 8 & $(188)$ \\
Iodination (mild) & $6-7$ & 4 & $(211)$ \\
Iodination (in crystals) & - & 4 & $(119)$ \\
Acetylation & 13 & 10 & $(88)$ \\
Glutarylation & 6 & - & $(112)$ \\
Succinylation & 9 & 10 1) & \\
Spectrophotometric titration & & & \\
\multicolumn{1}{c}{ pK $_{\text {int }}$ 9.2- 9.4 $9.9-10.3$} & 6.4 & 5.3 & $(119)$ \\
pK & 3.6 & 2.7 & $(69)$ \\
\hline Solvent perturbation (residues exposed) & 3 & 2 & \\
Solvent perturbation (residues exposed) & - & 6.4 & \\
\hline
\end{tabular}

1) Johansen, personal communication

tilisin type Carlsberg, GUNTELBERG and OTTESEN (59) demonstrated that the enzyme was almost completely inactivated by diisopropyl fluorophosphate (DFP). OTTESEN and SCHELlmanN (138) crystallized the DFPinactivated enzyme and demonstrated the incorporation of one gram of diisopropyl phosphate (DIP-) per 27.400 grams of enzyme, corresponding to a 1:1 stoichiometry (113). The inactivation of crystallized $\mathrm{BPN}^{\prime}$ with DFP, as described by MATSUbara and Nishimura (114), also involved 1:1 stoichiometry. Peptides containing the active site serine were isolated and the sequences determined by SANGER and SHAW (167) after partial acid hydrolysis of

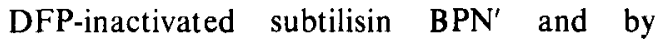
NOLLER and BERNHARD (131) after reaction with furylacryloylimidazole followed by enzymatic digestion. The sequence studies by SMITH and coworkers $(109,182)$ performed on the DIP-enzymes identified the seryl residue as $\mathrm{Ser}_{221}$ in both enzymes.

Almost complete inactivation of subtilisin BPN' $^{\prime}$ could be obtained with PMSF by reaction with $\operatorname{Ser}_{221}(129,147)$. Treatment of this derivative at $\mathrm{pH} \quad 5.2-5.5$ with thiolacetate expels the phenylmethylsulfonyl moiety with formation of a thiol ester which decomposes spontaneously to form "thiol" subtilisin according to the following scheme:

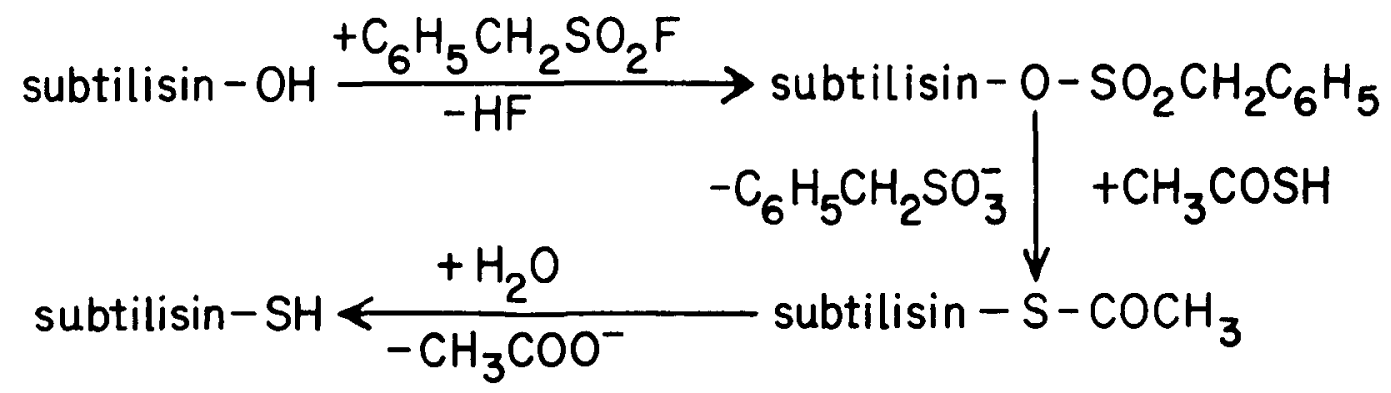


The subtle change of the OH-group of $\mathrm{Ser}_{221}$ to a SH-group led to loss of activity towards all amide and peptide substrates $(129,147)$ and ester substrates which do not have a very good leaving group. Subtilisin type Carlsberg is also inhibited by PMSF and can be further modified to the thiol derivative, the enzymatic consequences being the same as for the $\mathrm{BPN}^{\prime}$ enzyme (148).

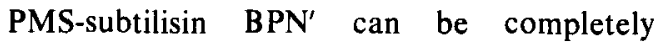
reactivated by incubation with formohydroxamic acid (206) while hydroxamic acid, being a weaker nucleophile, reactivates the enzyme to the extent of only $10 \%$ even after $15 \mathrm{hrs}$ of incubation at $\mathrm{pH}$ 9. Reactivation of the DIPenzyme was not possible with formohydroxamic acid and no reports showing reactivation of DIP-subtilisin have so far been published. A difference is noted here between subtilisin on the one hand and trypsin and chymotrypsin on the other, since the DIP-derivatives of the latter two enzymes have been reactivated by nucleophilic reagents $(33,34)$. MORRISETT and BROOMFIELD (128) explained the inertness of DIP-subtilisin to reactivation by steric inaccessibility of the reagent to the susceptible bond. If this explanation is correct, the observation by DRENTH and coworkers (40) that DIPsubtilisin $\mathrm{BPN}^{\prime}$ loses one isopropyl group upon crystallization is interesting from the view-point of reactivation. The loss of a bulky isopropyl group would reduce the steric barrier considerably and thus make the serylphospate bond more liable for attack by a nucleophilic reagent.

The inactivation of the subtilisins by KOCN most likely is due to carbamylation of $\operatorname{Ser}_{221}$ (187). Although direct evidence is still lacking for the subtilisins, the active seryl residue in chymotrypsin was shown to be carbamylated after reaction with radioactive labelled KOCN (176). Inactivation of chymotrypsin has also been achieved with p-nitrophenyl cyanate (161) and alkylisocyanates (26) and in both cases reaction with $\operatorname{Ser}_{195}$ was demonstrated. However, in these two cases the rate of inactivation was faster at high $\mathrm{pH}$ while the opposite was the case in the inactivation of subtilisin (187) and chymotrypsin by KOCN (176). A satisfactory explanation of this "abnormal " inactivation pattern is still lacking. The rate of inactivation of subtilisin $\mathrm{BPN}^{\prime}$ depended on a group with a $\mathrm{pK}_{\mathrm{a}} \mathrm{pf}$ about 7.7 and it was suggested (187) that a histidyl residue acted as the primary acceptor from which the carbamyl group was transferred to $\operatorname{Ser}_{221}$. However, such a reaction scheme was found most unlikely from model studies on carbamylation of imidazole by STARK (193). He suggested that it was the active seryl residue itself which acted as a nucleophile directly on cyanate. Since hydroxyamino acids react very poorly with cyanate (194) it was assumed that the high reactivity of $\operatorname{Ser}_{221}$ was mediated by a general base catalysis involving the histidine in the catalytic center. To the present author such an explanation does not account fully for the $\mathrm{pH}$ dependence of the inactivation. Since, according to STARK (194), the reaction will proceed via the following scheme:

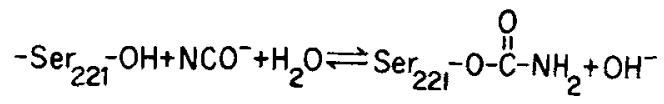

a decrease in $\mathrm{pH}$ will lower the concentration of both $\mathrm{NCO}^{-}$and activated $\mathrm{Ser}_{221}$ which in turn will lead to a drastic decrease in rate of reaction.

The reaction of HOCN with carboxylate groups depends on $\mathrm{pH}$ in a way compatible with the inactivation reaction of subtilisin and chymotrypsin:

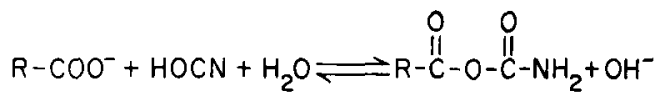

A decrease in $\mathrm{pH}$ will increase the forward reaction because more $\mathrm{HOCN}$ is available and decrease the backward reaction because of a decrease in $\mathrm{OH}^{-}(192)$. Carbamylcarboxylates are likely to be poor carbamylating reagents in solution but, since they are in a fixed position relative to the active seryl residue in the enzyme molecule, it is reasonable to assume that the reactivity might be greatly enhanced. However, the answer to this puzzling question must await further experimentation.

Inactivation of subtilisin has also been observed by reaction with hexamethylenediisocyanate (145). The inactivation in this case was also explained by reaction with $\operatorname{Ser}_{221}$. 


\subsubsection{Other Seryl Residues}

JOHANSEN (88) has shown that treatment of subtilisin type Carlsberg with succinic anhydride led to modification of about 12 seryl and threonyl residues. Using glutaric anhydride, it was possible to modify 14 residues. The drastic changes in enzymatic properties resulting from these reactions will be discussed in section 3.4.4.

\subsection{Modification of Histidyl Residues}

Inspection of the three-dimensional model of subtilisin BPN' shows that three of the six histidyl residues are deeply buried in the interior of the molecule. These are $\mathrm{His}_{39}, \mathrm{His}_{67}$ and $\mathrm{His}_{226}$. Although $\mathrm{His}_{17}$ is located at the surface of the molecule, it is oriented in such a way that protonation of the imidazole appears unlikely. Thus, only $\mathrm{His}_{64}$ and $\mathrm{His}_{238}$ are expected to respond normally to chemical modification and titration. Subtilisin type Carlsberg differs from the BPN' enzyme with respect to number and position of histidyl residues in the sequence, only in that $\mathrm{His}_{17}$ in the $\mathrm{BPN}^{\prime}$ enzyme is replaced by a glutamine residue in subtilisin type Carlsberg. The two enzymes should therefore respond similarly to chemical modifications of their histidines.

GOUNARIS and OTTESEN (56) suggested that some histidyl residues were located in the interior of subtilisin BPN'. Protonation of these residues would be one way of explaining denaturation of this enzyme at acid $\mathrm{pH}$. More direct physical-chemical evidence came from the potentiometric titration performed on the PMSF-inactivated enzyme by OTTESEN and RALSTON (144) who showed that the best agreement between the experimental data and a theoretical titration curve was obtained by assuming four buried histidyl residues and four buried carboxyls.

Chemical modification by means of photooxidation has been performed by OOSTERBAAN and COHEN (137) on subtilisin BPN'. One histidyl residue was modified, but the concomitant loss of activity could not be correlated with the loss of this residue alone, since the rate of inactivation was 1.7 times faster than the rate of disappearance of the histidyl residue. Simultaneous modification of one tryptophanyl and one methionyl residue probably increased the rate of inactivation. Although the oxidized histidyl residue was not identified it is reasonable to believe that it was the catalytically important $\mathrm{His}_{64}$. BISBY et al. (16) found that oxidation of one or more histidyl residues in the subtilisin by means of selective free radical reactions also led to inactivation.

The present author has shown that the histidyl residues of the subtilisins are unreactive toward the alkylating agents iodoacetate and iodoacetamide over a wide range of $\mathrm{pH}$ 's. In addition neither bromopyruvate nor bromoacetate react with the subtilisins (113).

Specific labelling of the histidyl residues in the catalytic center of trypsin and chymotrypsin has been obtained by alkylation with tosyl-Llysine chloromethyl ketone (TLCK) (177) and tosyl-L-phenylalanine chloromethyl ketone (TPCK) (177), respectively. The present author has shown that at $\mathrm{pH} 7.5$ and $30^{\circ} \mathrm{C}$, the conditions employed in the inactivation of chymotrypsin and trypsin, none of these active site directed reagents reacts with the subtilisins, probably due to poor binding. Similar results were obtained by SHAW and RUSCICA (178) who showed in addition that by substituting tosyl by benzyloxycarbinol in TPCK a slow inactivation of subtilisin $\mathrm{BPN}^{\prime}$ took place, and if the chloromethyl moiety was replaced by a bromomethyl moiety, the rate of inactivation proceeded with a reasonable rate $(50 \%$ inhibition in 4-5 hours). Subsequent isolation of the peptide containing the reactive histidyl residue showed this to be $\mathrm{His}_{64}$ (110). Although TLCK and TPCK do not react with $\mathrm{His}_{64}$, chloromethyl ketones of various peptides do, and such derivatives have been used in the kinetic and crystallographic studies of the secondary binding sites in subtilisin $\mathrm{BPN}^{\prime}$ as discussed in section 3.4.1.

\subsection{Modifications of Methionyl Residues}

Among the various methods available for chemical modification of methionyl residues $(32,208)$ oxidation by $\mathrm{H}_{2} \mathrm{O}_{2}$ has been applied to subtilisin type Carlsberg by STAUFFER and ETsON (195). Only one methionyl residue was found to be modified. Following denaturation and digestion with trypsin, a peptide was 
isolated which contained the methionine sulfoxide formed by the oxidation. This residue was identified as $\mathrm{Met}_{222}$, located next to the active $\operatorname{Ser}_{221}$.

Kinetic studies showed that the oxidized enzyme was still partly active towards various substrates. While $\mathrm{K}_{\mathrm{M}}$ (app) changed to a minor degree only (both decreases and an increase were observed), $k_{\text {cat }}$ decreased by a factor ranging from 2 (cinnamoyl imidazole) to 10 (acetyl tyrosine ethyl ester). According to STAUFFER and ETSON (195), the presence of the sulfoxide dipole of oxidized methionine near the active seryl and histidyl residues will alter the electronic configuration in such a way as to change the rate of hydrolysis. In the case of trans-cinnamoyl imidazole hydrolysis, it was possible to resolve $\mathrm{k}_{\text {cat }}$ into $\mathrm{k}_{\text {acylation }}$ and $\mathbf{k}_{\text {deacylation. }}$ It was shown that the deacylation step was affected to the largest extent.

As already mentioned (section 2.4) inactivation of subtilisin $\mathrm{BPN}^{\prime}$ by photooxidation led to modification of one methionyl residue (137). However, the modified methionyl residues were shown not to be $\mathrm{Met}_{222}$ in this case.

Finally, N-bromosuccinimide (NBS) has been used in modification studies on subtilisin

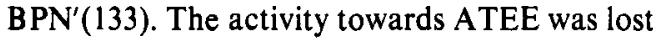
concomitantly with modification of methionyl, tyrosyl and tryptophanyl residues. At low degree of modification (2-4 moles of NBS per mole of enzyme), predominantly a methionyl and a tyrosyl residue were modified with great loss of activity suggesting that the main reason for inactivation by this reagent is due to modification of a methionyl residue. It appears less likely that the modification of a single tyrosyl residue will give rise to loss of activity towards ATEE, since no tyrosyl residues are located in the catalytic region.

\subsection{Modification of Tryptophanyl Residues}

As mentioned above (section 2.4) one tryptophanyl residue in subtilisin $\mathrm{BPN}^{\prime}$ was modified by photo-oxidation (137). Oxidation with a 10 -fold excess of $\mathrm{N}$-bromosuccinimide also led to modification of one residue (133). However, changes in other residues took place as well. The specific tryptophanyl reagent 2hydroxy-5-nitrobenzylchloride (Koshland's rea- gent I) reacts with subtilisin $\mathrm{BPN}^{\prime}$ only to a very limited extent. The present author found that only 0.1 mole of Trp per mole of enzyme was modified at $\mathrm{pH} 4.75$ and a molar excess of reagent of 500, when estimated spectroscopically. The result is in agreement with that of NEET and Koshland (129). The water soluble modification of Koshland's reagent I: dimethyl (2-methoxy-5-nitrobenzyl) sulfonium bromide did not react with subtilisin BPN' either, showing that the lack of reaction with the tryptophanyl residues was not due to the poor solubility of Koshland's reagent I. However, it was found that in acid denatured enzyme approximately two out of the three possible residues in subtilisin BPN' were modified.

The difference in the rates of inactivation of the two subtilisins by $\mathrm{H}_{2} \mathrm{O}_{2}$-dioxane was demonstrated by the present author in the following way: To a $800 \mu$ l solution containing $5 \mathrm{mg}$ subtilisin type Carlsberg or subtilisin BPN' $^{\prime}, 200 \mu \mathrm{l}$ peroxide-free dioxane, $2 \mathrm{ml} \mathrm{l} \mathrm{M}-\mathrm{NaHCO}_{3}$, and $800 \mu \mathrm{l} \mathrm{H}_{2} \mathrm{O}$ was added $50 \mu \mathrm{l}$ of a $3 \% \mathrm{H}_{2} \mathrm{O}_{2}$ solution. The reaction mixture was left at room temperature and $25 \mu$ laliquots were removed every $15 \mathrm{~min}$ for activity assays on $0.5 \%$ clupein sulphate, using the $\mathrm{pH}$-stat technique. As seen in Fig. 2, both enzymes lost their activity toward

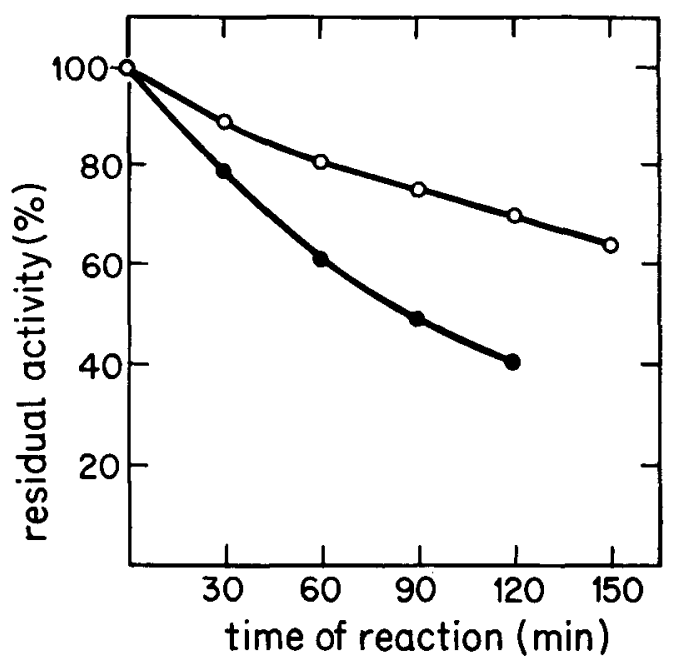

Figure 2. Inactivation of subtilisin type Carlsberg -O- and subtilisin $\mathrm{BPN}^{\prime}-$ by $\mathrm{H}_{2} \mathrm{O}_{2}$ in a dioxane- $\mathrm{NaHCO}_{3}$ mixture at room temperature. Clupein sulfate was used as substrate for activity assays. 
clupein, but the rate of inactivation was much faster with subtilisin $\mathrm{BPN}^{\prime}$ than with subtilisin type Carlsberg.

According to HASHIMORI et al. (65) from whom the experimental procedure used above was adopted, the extent of modification of tryptophanyl residues can be followed by the decrease in absorbance at $282 \mathrm{~nm}$. Although the characteristic isosbestic points at $267 \mathrm{~nm}$ and $297 \mathrm{~nm}$ were observed in the present experiments, the total decrease in absorbance at $282 \mathrm{~nm}$ was larger than could be accounted for by modification of trytophanyl residues alone. An estimation of the degree of modification of methionyl residues has not been made in the present experiments, and it is therefore not possible to explain the cause of inactivation. The difference in rate of inactivation may, however, reflect a difference in stability of the two enzymes, especially since the BPN' enzyme contains three and the Carlsberg enzyme only one tryptophan per molecule.

From solvent perturbation studies using 20\% ethylene glycol MYERS and GLAZER (119) estimated the number of exposed tryptophanyl residues in subtilisin BPN' to 1.6 and in the Carlsberg enzyme to 0.8 . A similar result was obtained by HERSKOVITS and FUCHS (69). Using eight different perturbants the average value for exposed residues in subtilisin $\mathrm{BPN}^{\prime}$ was found to be $1.9 \pm 0.3$. In $8 \mathrm{M}$-urea all three residues were freely exposed.

Although the three tryptophanyl residues in subtilisin $\mathrm{BPN}^{\prime}$ and the single residue in the Carlsberg enzyme are located near the surface of the enzyme molecule, they are still partially buried. Trp ${ }_{106}$ in subtilisin BPN' was estimated from the three-dimensional model to be only $30 \%$ exposed while the corresponding values for $\operatorname{Trp}_{113}$ and $\operatorname{Trp}_{241}$ were $30-40 \%$ and $50-70 \%$, respectively. If the estimation of the accessibility of these residues in the crystalline state also is valid for the enzyme in solution, the solvent perturbation experiments seem to overestimate the degree of exposure while the inertness of these residues towards Koshland's reagent becomes more understandable. Studies with model compounds indicate that the alkylation takes place at $\mathrm{C}-3$ in the indole ring (108). In a protein this would be close to the "back- bone" of the peptide chain which usually is less exposed than the side-chains:

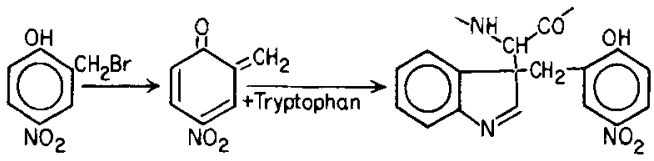

However, it must be remembered that the size of the reagent may be partly responsible for the lack of reaction with tryptophan.

\subsection{Modification of Glutamic and Aspartic Acid Residues}

ADAMS (3) has modified the free carboxyl groups in subtilisin $B P N^{\prime}$ according to the "carbodiimide method" of HOARE and Koshland (72). It was found that when the native enzyme was treated with $\mathrm{N}$-ethyl- $\mathrm{N}^{\prime}-1,3-$ dimethyl aminopropyl carbodiimide (EDC) in the presence of glycineamide or glycinemethyl ester at $\mathrm{pH}$ 5.1, the maximum number of modified carboxyl groups was eight, while all 16 groups reacted in the acid denatured enzyme. The lack of reactivity of eight carboxyl groups in the native enzyme may, in part, be explained by inaccessibility of the reagent as judged from an inspection of the three-dimensional model. It was also noted that four carboxyl groups were close to either lysyl or arginyl side-chains and could possibly form salt-bridges which in turn would decrease the reactivity of the carboxyl groups. However, it appears to the present author that such salt-bridges must be weak, since they are formed in aqueous surroundings. It therefore appears likely that the reactivity of the salt-bridged carboxyl groups should be affected only to a minor extent.

ADAMS (3) has discarded the possibility that the enzyme denatures if more than eight groups are modified because the yield of modified enzyme was independent of the amount of EDC used. No stability experiments have been performed with the modified enzyme, but a smear was seen in polyacrylamide gel electrophoresis of the maximally modified enzyme, indicating that this is more unstable than the native or lightly modified enzyme.

SVENDSEN and STACHOWITZ have attempted to modify subtilisin type Carlsberg under the following conditions: $\mathrm{pH} 4.75,3^{\circ}$, glycine 
methyl ester $(600 \mathrm{mg}), \mathrm{N}$-cyclohexyl- $\mathrm{N}^{\prime}[\beta-(\mathrm{N}-$ methyl morpholino) ethyl] -carbodiimide (CMC) (225 mg), enzyme ( $25 \mathrm{mg}$ ) in $7 \mathrm{ml} \mathrm{H}_{2} \mathrm{O}$ for $20 \mathrm{~min}$. No incorporation of glycine was observed when an amino acid analysis was performed on a gel filtered, acid hydrolyzed sample. On the other hand, acid denatured subtilisin type Carlsberg incorporated eight glycine residues (out of possible 13) under the same conditions. The failure of a quantitative reaction is most likely due to the omission of guanidine hydrochloride from the reaction mixture thereby preventing complete random coil formation.

Since nine of the carboxyl residues are located in identical positions in the two subtilisins it appears quite unlikely that their lack of reaction in subtilisin type Carlsberg is caused by molecular differences between the two enzymes. Since $\mathrm{CMC}$ is a bigger and more "clumsy" molecule than EDC it seems plausible that steric hindrance is the main reason for the lack of reactivity of CMC. It is possible that if a carbodiimide smaller than EDC could be used, more than eight carboxyl groups would react in subtilisin BPN'. The results discussed above are a warning against too categorical conclusions about "free" and "buried "functional groups in proteins.

The interesting enzymatic consequences which result from modifying the carboxyl groups in subtilisin BPN' will be discussed in section 3.4.4.

\section{BINDING SITES OF THE SUBTILISINS}

\subsection{Definitions}

In this discussion of the binding of peptide substrates to the subtilisins it is useful to first define the terms related to the functioning of the enzyme as they are used in this thesis. It is apparent from the literature that different terms sometimes are used to express the same concept.

The active site is that part of the enzyme which is involved in both the binding and hydrolysis of substrate.

The catalytic site consists of those residues which are directly involved in the breakage of peptide or ester bonds. In the subtilisins the catalytic site consists of $\operatorname{Ser}_{221}, \mathrm{His}_{64}$, and possibly $\mathrm{Asp}_{32}$ (see section 3.2).

The binding site for a given substrate consists of that part of the enzyme surface which participates in the formation of the Michaëlis complex between enzyme and substrate. As will be shown in this and the following section, the binding sites of proteolytic enzymes can be large and it is convenient therefore to divide it into subsites. The term "subsite" was originally defined by SCHECHTER and BERGER (170) as the region on the enzyme surface which interacts with one amino acid of the substrate ". The nomenclature for numbering the subsites introduced by the authors mentioned above will be used in the following and is illustrated by Fig. 3. The arrow indicates the bond which is hydrolyzed. $S_{1}$ and $S_{1}^{\prime}$ are subsites 1 and $1^{\prime}$,

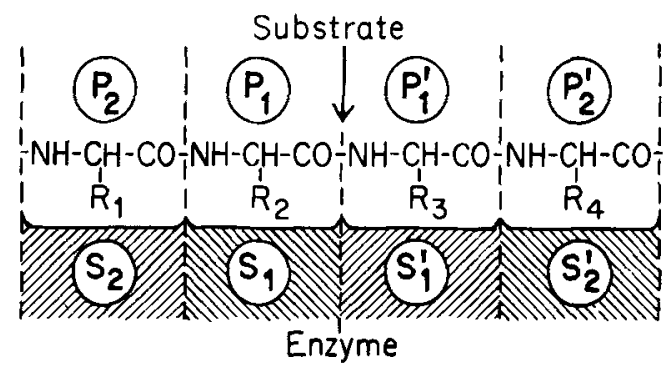

Figure 3. Binding between enzyme and substrate according to the subsite nomenclature of SCHECHTER and BERGER (170). The arrow indicates the bond to be hydrolyzed. $S_{1}$ and $S_{2}$ are subsites on the enzyme binding amino acid residues $P_{1}$ and $P_{2}$ located on the $N$-terminal side of the susceptible bond. $S_{1}^{\prime}$ and $S_{2}^{\prime}$ are subsites binding amino acid residues $P_{1}^{\prime}$ and $P_{2}^{\prime}$ on the $\mathrm{C}$-terminal side of the bond to be hydrolyzed. For further details see text.

respectively, which accept the two amino acid residues, $P_{1}$ and $P_{1}^{\prime}$, that donate a carbonyl and amino group, respectively, to the susceptible bond. The subsites are numbered $S_{1}, S_{2}, \ldots S_{n}$ towards the $\mathrm{N}$-terminal end of the substrate and $S_{1}^{\prime}, S_{2}^{\prime}, \ldots S_{n}^{\prime}$ towards the C-terminal end. The primary binding sites are subsites $S_{1}$ and $S_{1}^{\prime}$ and the secondary binding sites are subsites $S_{2}$ to $S_{n}$ and $S_{2}^{\prime}$ to $S_{h}^{\prime}$, respectively. As seen from Fig. 3, a subsite accomodates both the side-chain and backbone part (-NH-CH-CO-) of a substrate amino acid residue. If the $\mathrm{N}$ - and $\mathrm{C}$ - 
terminal ends of the substrate are blocked, the blocking groups are potential occupiers of subsites of their own. Therefore, the term P should not be restricted to amino acid residues alone, but also include possible blocking groups although these were not included in the original definition by SCHEChTER and BERGER. Disregard of the effect of blocking groups may lead to wrong interpretations of the effect of chainelongation on the hydrolysis of synthetic peptides.

Specificity. Operationally, specificity can be defined as kinetic specificity which is the "dependence of $k_{\text {cat }}$ on the structure " (46) and as binding specificity which is the mselectivity shown by an enzyme in the formation of an enzyme-substrate complex" (46). Such selectivities are expressed in the preference of the enzyme for one type of side-chain over others, side-chain specificity, or in the preference for one of the stereoisomers (usually the L-form of an amino acid), stereochemical specificity.

Recognizing that in large substrates multiple interactions between substrate and protein took place, NeIL et al. (130) distinguished between primary specificity which was the minimum structure required for enzymatic specificity to be manifest " and secondary specificity which is determined by the influence of all other interactions between enzyme and substrate on the hydrolytic action. In the case of the highly specific trypsin, the term "specificity site" seems appropriate, but since most other enzymes, including the subtilisins, hydrolyze a variety of bonds, all the interactions between enzyme and substrate must be taken into consideration in evaluating the specificity. Such a view, recently advocated by FRUTON (46) in the case of pepsin, also holds true for the subtilisins as will be apparent from the following discussion.

Binding via secondary sites is not restricted to polypeptides alone, but may occur whenever hydrolyses of polymer compounds are taking place. Lysozyme serves as a relevant example of a carbohydrate hydrolyzing enzyme in which six subsites have been recognized $(90,164)$. Nine to ten binding sites were recognized in amylase from Bacillus subtilis (162) while five were seen in $\alpha$-amylase from pig (163) and six in glucoamylase from Rhizopus delemar (71). Multiple subsites have been recognized in the interaction between oligonucleotides and polynucleotide phosphorylase (30) and staphylococcal nuclease (35). No reports have been published on secondary binding sites of lipases. It is reasonable to assume, however, that the binding of the polymeric lipids takes place also via secondary bindings.

\subsection{Mechanism of Action of the Subtilisins}

The hydrolysis of peptide and ester bonds by the subtilisins (and other serine proteases as well) proceeds via an enzyme-substrate intermediate in which a covalent bond is formed between the carboxyl group of the susceptible bond in the substrate and the seryl residue in the catalytic site (e.g. 12): esterification, alcoholysis) or the $\alpha-\mathrm{NH}_{2}$ group of peptides (trans-peptidation, ammoniolysis) can also serve as nucleophiles attacking the acylated enzyme E-CO-R. Trans-esterification has been demonstrated in the action of subtilisin type Carlsberg on L-tyrosine ethyl ester (52), while no trans-peptidation has been reported with the subtilisins (113). However, the

$$
\begin{aligned}
& \mathrm{E}-\mathrm{H}+\mathrm{R}-\mathrm{CO}-\mathrm{X} \stackrel{\mathrm{K}}{=} \mathrm{S} \rightleftharpoons \mathrm{E}-\mathrm{H} \cdot \mathrm{R}-\mathrm{CO}-\mathrm{X} \stackrel{\mathrm{k}_{2}}{\longrightarrow} \mathrm{E}-\mathrm{CO}-\mathrm{R}+\mathrm{HX} \text { (acylation) } \\
& \mathrm{E}-\mathrm{CO}-\mathrm{R}+\mathrm{H}_{2} \mathrm{O} \stackrel{\mathrm{k}_{3}}{\longrightarrow} \mathrm{E}-\mathrm{H}+\mathrm{R}-\mathrm{CO}-\mathrm{OH} \text { (deacylation) }
\end{aligned}
$$

$\mathrm{K}_{\mathrm{s}}$ is the dissociation constant for the Michaëlis complex, $k_{2}$ and $k_{3}$ are the rate constants for acylation and deacylation of the enzyme, respectively. The deacylation step normally involves $\mathrm{H}_{2} \mathrm{O}$ (hydrolysis), but alcohols (trans- less than quantitative recovery of identifiable hydrolysis products following digestion of the B-chain of oxidized insulin by the subtilisins (85) can be interpreted as being due to transpeptidase action which gives rise to an 
indeterminable "back-ground " of peptides. The catalytic parameters $\mathrm{K}_{\mathrm{M}}$ (app) and $\mathrm{k}_{\text {cat }}$ are related to the constants referred to above in the following way:

$$
\begin{aligned}
& K_{M}(a p p)=K_{s} \frac{k_{3}}{k_{2}+k_{3}} \\
& k_{\text {cat }}=\frac{k_{2} k_{3}}{k_{2}+k_{3}}
\end{aligned}
$$

If the rate limiting step is acylation $\left(\mathbf{k}_{2} \ll \mathbf{k}_{3}\right)$ it follows that $\mathrm{K}_{\mathrm{M}}(\mathrm{app})=\mathrm{K}_{\mathrm{s}}$, which means that the measured dissociation constant is identical to the true dissociation constant of the Michaëlis complex and $k_{\text {cat }}=k_{2}$. This is generally accepted to be the case in hydrolysis of peptide bonds. However, in hydrolysis of esters $k_{3} \ll k_{2}$, which makes deacylation the rate limiting step, and in this case $k_{\text {cat }}=k_{3}$, and $K_{M}(a p p)=K_{s} \frac{k_{2}}{K_{3}}$ and hence can be very far from the true dissociation constant.

Both $K_{M}$ (app) and $k_{c a t}$ depend on differences between $k_{2}$ and $k_{3}$. However, dividing equation (2) by (1) gives

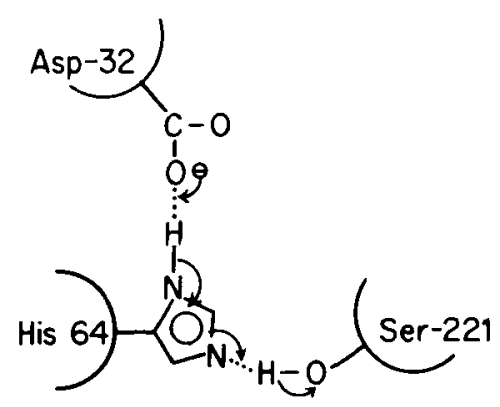

$$
\frac{\mathrm{k}_{\mathrm{cat}}}{\mathrm{K}_{\mathrm{M}}(\mathrm{app})}=\frac{\mathrm{k}_{2}}{\mathrm{~K}_{\mathrm{s}}}
$$

which shows that $k_{\text {cat }} / K_{M}(a p p)$ is a true measure of catalytic efficiency. This ratio is therefore used in comparative kinetic studies when the individual rate constants $k_{2}$ and $k_{3}$ cannot be obtained.

Although the participation of $\operatorname{Ser}_{221}$ and $\mathrm{His}_{64}$ in the catalytic step (see sections 2.3 .1 and 2.4 ) is well documented, a satisfactory explanation of the unusual reactivity of $\operatorname{Ser}_{221}$ has been lacking. However, BLow et al. (19) suggested the participation of an aspartic acid residue buried in the hydrophobic interior of $\alpha$-chymotrypsin, but oriented in such a way that hydrogen-bond formation to the histidyl residue in the catalytic site was readily established. Through a hydrogen bond system, electrons can fluctuate between this aspartic acid residue and the hydroxyl groups of the active seryl residue. This so called "charge relay system " has also been suggested for the subtilisins based on the observation that the spatial orientation of $\mathrm{Ser}_{221}, \mathrm{His}_{64}$ and $\mathrm{Asp}_{32}$ is identical to the one found in chymotrypsin (216):

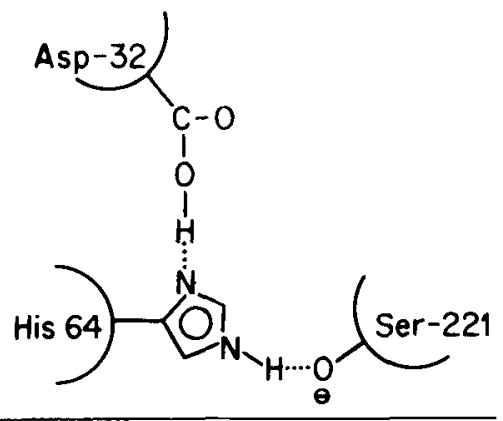

The enzymatic activity of the subtilisins depends on at group with a $\mathrm{pK}_{\mathrm{a}}$ of about 7 , and $\mathrm{His}_{64}$ has until recently been accepted as that group. However, HUNKAPILLER et al. (79) have shown that in $\alpha$-lytic protease (which is a bacterial serine protease), an aspartic acid

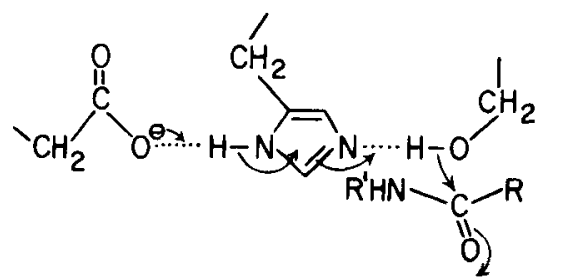

residue rather than the sole histidyl residue in this enzyme has a $\mathrm{pK}_{\mathrm{a}}$ of 6.7 - in fact the $\mathrm{pK}$ of the histidyl residue was lower than 4 . The following mechanism for the operation of the "charge relay system" in serine proteases was proposed for the acylation step: 
The tetrahedral intermediate breaks down to the acylated intermediate expelling the leaving group NHR'. In the deacylation step the sequence is reversed by nucleophilic attack of $\mathrm{H}_{2} \mathrm{O}$.

According to WOLFENDEN (214) the binding of substrate is much stronger in the transition state than in the Michaelis complex. Kraut and coworkers (160) have shown from modelbuilding experiments with subtilisin $\mathrm{BPN}^{\prime}$ that in the tetrahedral intermediate the carboxyl oxygen of the hydrolyzing bond can form two hydrogen bonds to amido groups in the enzyme in the so-called "oxyanion hole" and an additional hydrogen bond can be formed between the $P_{1}$ amido group and the enzyme. Thus, the transition state is stabilized relative to the Michaëlis complex and the acyl intermediate by a decrease in free energy due to the formation of three hydrogen bonds, which in turn leads to an increase in acylation rate.

Transition state analogs, which should give useful information about the correctness of a postulated transition state, have been studied in a couple of cases to verify the existence of a tetrahedral intermediate in catalysis by serine proteases. Thus THOMPSON (199) has shown that peptide aldehydes of good elastase substrates bind very strongly to the enzyme. For example, $\mathrm{K}_{\mathrm{I}}$ for Ac-Pro-Ala-Pro-Alaninal is $8 \times 10^{-7} \mathrm{M}$ at $\mathrm{pH} 7.0$. The reason for the strong binding is believed to be the existence of a tetrahedral addition complex with the catalytic active serine:

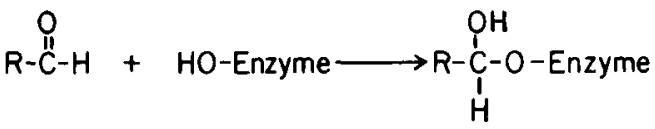

In this connection it is of interest to note (199) that a novel class of protease inhibitors, the leupeptins, were shown to be peptide aldehydes (7).

A possible transition state analog for chymotrypsin, the inhibitor 2-phenylethane boronic acid, was studied by KOEHLER and LIENHARD (96) who found a binding constant of $4 \times 10^{-5} \mathrm{M}$ at $\mathrm{pH} 8$. The rationale behind the choice of a boronic acid derivative was the tendency of boron to form tetrahedral adducts. The follow- ing addition product was visualized for chymotrypsin:<smiles></smiles>

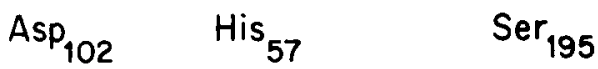

Subtilisin BPN' is inhibited to the same extent by p-bromobenzene boronic acid $\left(\mathrm{K}_{\mathrm{I}}=\right.$ $\left.2.27 \times 10^{-5} \mathrm{M}(146)\right)$. The degree of inhibition, which increases with $\mathrm{pH}$ depends on a group with a $\mathrm{pK}$ of about 7 . This group was assumed to be $\mathrm{His}_{64}$ and two possible reactions (A and B) were considered:

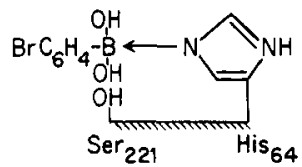

(A)

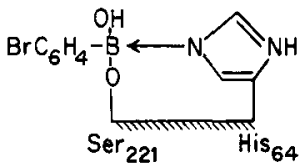

(B)
(A) being favored because the reaction was very fast. However, in a recent study of the inhibition of subtilisin type Carlsberg by various boronic acids LINDQUIST and TERRY (106) concluded that the formation of a covalent bond between $\operatorname{Ser}_{221}$ and boronic acid was more appealing. This assumption has been strengthened by $\mathrm{X}$-ray diffraction studies by KRAUT and coworkers (115) who showed that tetrahedral adducts were formed when benzeneboronic acid or 2-phenylethaneboronic acid were bound to crystals of subtilisin BPN', $^{\prime}$ and that a covalent bond was formed to $\operatorname{Ser}_{221}$. Tetrahedral adducts were also formed when arylsulfonic halides and arsonic acids were bound to crystals of subtilisin BPN' (15). GLAZER $(54,55)$ has studied the inhibition of both subtilisins by various arsonic acids (e.g. phenylarsonic acid) and found that the inhibition was time-dependent and also depended on a group with a $\mathrm{pK}_{\mathrm{a}}$ of 7.2 being most effective in the protonated state. This group was assumed to be $\mathrm{His}_{64}$, but in view of the recent finding by HUNKAPILLER et al. (79) mentioned above that the histidine probably has a $\mathrm{pK}_{\mathrm{a}}$ value lower than 4 also in the subtilisins, an alternative explanation must be sought. The time-dependent 
inactivation of the subtilisins by arsonic acids (not observed with chymotrypsin) also remains to be explained.

\subsection{Specificity}

\subsubsection{Synthetic substrates}

The specificity of the subtilisins is broad. It was shown by GLAZER (53), BAREL and Glazer (11) and Morihara and TsUzUKI (122) that esters of various $\mathrm{N}$-terminal blocked amino acids were hydrolyzed, but with different rates. The enzymes hydrolyzed esters of aromatic amino acids considerable faster that those of aliphatic amino acids. This finding suggested that $S_{1}$ in the subtilisins was hydrophobic, and $\mathrm{X}$-ray crystallographic studies have later confirmed this suggestion. A hydrophobic "groove" rather than a "pocket" was observed probably reflecting the broader specificity of the subtilisins compared to that of chymotrypsin and trypsin (159).

A comparison of the Carlsberg and $\mathrm{BPN}^{\prime}$ enzymes showed that $V_{\max }$ values for aromatic ester substrates was higher for subtilisin type Carlsberg than for subtilisin BPN'. Since acylation proceeds considerably faster than deacylation in the hydrolysis of ester substrates (12), the differerence in $V_{\max }$ probably reflected a difference in rate of deacylation. This suggestion was later strengthened when JOHANSEN $e t$ al. (87) demonstrated that the deacylation of $\mathrm{N}$ trans-cinnamoyl imidazole and the indoleacryloyl intermediates of the subtilisins is faster for the Carlsberg enzyme. These authors also demonstrated the same difference in rate of deacylation in chemically modified subtilisins. Thus, nitration, iodination, glutarylation and succinylation have no effect, supporting other evidence that lysyl and tyrosyl residues do not participate in the hydrolysis of small ester substrates.

The esterase activity of the subtilisins is emphasized by the observations of GUNTHELBERG and OTTESEN (59) who showed that methylbutyrate was hydrolyzed by subtilisin type Carlsberg, and by GRAAE (57) who found that the same enzyme hydrolyzed esters of fatty acids with chain-lengths ranging from three to eight carbon atoms. Methyl valerate was found to be the best substrate, further elongation of the chain led to a decrease in the rate of hydrolysis.

MORIHARA and TsUzUKI (122) followed the deamidation of a series of dipeptides of the type Z-Gly-X-NH $\mathrm{N}_{2}$ by subtilisin BPN'. Although the results could be evaluated on a qualitative basis only, they showed that $\mathrm{X}=\mathrm{Tyr}$ resulted in the fastest rate of hydrolysis, but dipeptides containing Phe or Leu in $P_{1}$ were also hydrolyzed at a reasonable rate. It appears therefore, that at least for dipeptides the same specificity pattern holds as for esters: a preference for aromatic or hydrophobic side-chains. Stereospecificity of subsite $S_{1}$ was demonstrated in experiments were L-leucine was substituted by $D$ leucine.

ERLANGER and COHEN (42) showed that diphenylcarbamylchloride (DPCC) inactivates chymotrypsin about 75 times faster than trypsin, and a method for "all-or-none assay of chymotrypsin was developed using a chromophoric variant, 2-nitro-4-carboxyphenyl-N,Ndiphenylcarbamate (NCDC) (43). Both reactants reacted with chymotrypsin in a stoichiometric ratio of 1:1 with concomitant inactivation. Because of the similarities between the subtilisins and chymotrypsin in their specificity towards synthetic ester substrates an attempt was made by the present author to use NCDC in an "all-or-none" assay for subtilisins. However, both DPCC and NCDC proved to be fairly good substrates for the subtilisins and reliable estimations of enzyme concentration from the "burst « or presteady-state reaction was not possible. It was shown that at $\mathrm{pH} 9.1$ the hydrolysis of NDCD (initial conc. $1.7 \times 10^{-3} \mathrm{M}$ ) by subtilisin type Carlsberg (initial conc. $7.9 \times 10^{-5} \mathrm{M}$ ) was about three times faster than that of subtilisin $\mathrm{BPN}^{\prime}$ (same conc.). This result emphazises the quantitative differences in specificity towards ester substrates which exist between the two enzymes.

\subsubsection{Natural Peptides and Proteins}

In their action on peptides and proteins, the subtilisins show a very broad specificity, which is the reason for the utility of these enzymes in sequence work.

The B-chain of oxidized insulin is the most commonly used peptide for determining the 
(I) $\mathrm{CySO}_{3} \mathrm{H}-\mathrm{Tyr}-\mathrm{Ile}-\mathrm{Gln}-\mathrm{Asn}-\mathrm{CySO}_{3} \mathrm{H}-\mathrm{PrO}-\mathrm{Leu}-\mathrm{G} \ln (205)$

(II) His-Ser-Gln-Gly-Thr-Phe-Thr-Ser-Asp-Tyr-Ser-Lys-Tyr-Leu-Asp-Ser-Arg-Arg-Ala-Gln-Asp-Phe-Val-Gln-Try-Leu-Met-Asp-Thr (181)

(II) Ile-Ile-Glu-Val-Glu-Asn-Gln-Ala-Asn-Pro-Thr-Thr-Ala-Glu-Thr-Leu-Asp-Ala-Thr-Arg (221)

$\uparrow \uparrow \uparrow \uparrow$

(D) Phe-Val-Asn-GIn-His-Leu-CySO 3 H-Gly-Ser-His-Leu-Val-Glu-Ala-Leu-Tyr-Leu-Val-CySO 3 H-Gly-Glu-Arg-Gly-Phe-Phe-Tyr-Thr-Pro-Lys-Ala

$\begin{array}{llllllllllllllllll}a & \uparrow & \uparrow & \uparrow & \uparrow & \uparrow & \uparrow & \uparrow & \uparrow & \uparrow & \uparrow & \uparrow & \uparrow & \uparrow & \uparrow & \uparrow & (204)\end{array}$

b $\quad \uparrow \uparrow \uparrow$

$\uparrow \uparrow$

c

$\uparrow$

d 1

e $\uparrow$

$\uparrow$

$\uparrow$

$\uparrow \uparrow$

$\uparrow \uparrow \uparrow \uparrow$

$\uparrow \uparrow \uparrow$

$\uparrow$

$\begin{array}{lll}\uparrow & \uparrow \\ & & \uparrow \\ & & \uparrow \\ & & \uparrow \\ & & \uparrow\end{array}$

Figure 4. Hydrolysis of various naturally occuring polypeptides by the subtilisins. The arrows indicate the bonds that are split. (I) Oxytocin, (II) glucagon, (III) tryptic peptide from tobacco mosaic virus, (IV) B-chain of oxidized insulin. In IVb and $c$ insulin with its disulfide bonds intact was used. For further details see text.

specificity of proteolytic enzymes and the earliest investigations of subtilisin type Carlsberg were performed on this peptide. Such results are shown in Fig. 4, IVa-e. The works of TupPy (204), HaugaARD and HaugaARD (66), and MEEDOM (118) all have been designed to give as complete a hydrolysis as possible (Fig. 4, IV a-c) and indeed a high percentage of the bonds were hydrolyzed. It should be noted that $\mathrm{IVb}$ and $\mathrm{c}$ were performed on insulin with the disulfide bridges intact. This does not appear to have any profound influence on the availability of susceptible bonds. A more complete hydrolysis of the B-chain of oxidized insulin (IV a), was observed, but in this case a longer incubation period and a higher enzyme concentration was used. However, the difference in the resulting peptide pattern might also reflect a difference in availability of bonds in the two cases. Experiments designed to elucidate the initial steps in the hydrolysis of the oxidized Bchain of insulin are shown in Fig. 4, IV d and e. It is evident that the number of bonds which are rapidly hydrolyzed is very restricted $(85,122)$. Of special interest for the present discussion was the finding by JOHANSEN et al. (85) that the bond $\mathrm{Leu}_{15}-\mathrm{Tyr}_{16}$ was hydrolyzed much faster than any of the other bonds in the molecule. The specificity requirement deduced from hydrolysis of small synthetic substrates (3.3.1) would suggest hydrolysis between $\mathrm{Tyr}_{16}$ and $\operatorname{Leu}_{17}$. The result strongly suggested that the secondary binding sites of the subtilisin type Carlsberg played an important role in this case.
This suggestion will be further elaborated in the general discussion of the secondary binding sites in the subtilisins (see section 3.2.4).

Both subtilisins have been used for hydrolysis of the B-chain of oxidized insulin (85). While no qualitative differences could be observed (both enzymes hydrolyzed the same bonds) quantitative differences were noted. Thus the bonds $\mathrm{Ser}_{9}-\mathrm{His}_{10}$ and $\mathrm{Tyr}_{26}-\mathrm{Thr}_{27}$ were hydrolyzed faster with subtilisin $\mathrm{BPN}^{\prime}$, while the opposite was the case with the bonds $\mathrm{Gln}_{4}-\mathrm{His}$, and $\mathrm{Leu}_{11}-\mathrm{Val}_{12}$.Some of the earliest investigations of the specificity of subtilisin BPN' showed a preference for acidic amino acids. This was found by TAZAWA (197) using ovalbumin and gelatine. However, UKUNUKO et al. (135) using oxidized lysozyme and oxidized $\alpha$-amylase as substrates found no specificity differences between neutral and acidic side-chains while only weak hydrolysis was found for bonds involving aromatic and basic amino acids residues. TUPPY (205), on the other hand, found that oxidized oxytocin was hydrolyzed on the C-terminal side of Gln and Leu (Fig. 4, I) by subtilisin type Carlsberg. This enzyme was also used in determining the sequence of glucagon (181) (Fig. 4, II). The effective hydrolysis of an ArgArg bond is noteworthy in this case. The further degradation of a tryptic peptide from tobacco mosaic virus protein by subtilisin $\mathrm{BPN}^{\prime}$ is shown in Fig. 4, III (221). A big difference was observed between the subtilisins in their action on $\mathrm{N}, \mathrm{N}$-dimethylcasein (61). Finger-prints of the liberated peptides showed that only 20 
peptides out of 60 liberated were common for subtilisin type Carlsberg and subtilisin BPN'. $^{\prime}$.

Limited proteolysis was observed in the classical experiment of OTTESEN (139) in which subtilisin type Carlsberg transformed ovalbumin into plakalbumin by release of an internal hexapeptide. The same two bonds were hydrolyzed in ovalbumin by both subtilisins, but differences were noted in the further breakdown of the hexapeptide (140). Another example of limited proteolysis is the hydrolysis of DNA polymerase by subtilisin type Carlsberg which eliminates the exonuclease activity while the polymerase activity is still intact $(29,95)$. Carboxypeptidase A can under appropriate conditions be hydrolyzed by subtilisin type Carlsberg in such a way that only two peptide bonds are broken (158). However, the enzymatic properties of the product, which is called carboxypeptidase $S$, are altered in such a way that its esterase activity has increased $4-5$ fold while the peptidase activity decreases to one half that of the native enzyme.

A qualitative difference may exist between the subtilisins in their action on ribonuclease. Under suitable chosen conditions only one bond is hydrolyzed by subtilisin type Carlsberg (153), namely the one between, $\mathrm{Ala}_{20}-\mathrm{Ser}_{21}$ in the sequence:

$-\operatorname{Thr}_{17}-\operatorname{Ser}_{18}-\mathrm{Ala}_{19}-\mathrm{Ala}_{20}-\operatorname{Ser}_{21}-\operatorname{Ser}_{22}-\mathrm{Ser}_{23}-\mathrm{Asn}_{24}-$

Subtilisin BPN', on the other hand, cleaved two bonds, those between $\mathrm{Ala}_{20}-\mathrm{Ser}_{21}$ and $\mathrm{Ser}_{21}$ $\mathrm{Ser}_{22}$, respectively (58). The S-peptides 1-20 and $1-21$ were isolated in the ratio $2: 1$. The finding that the peptides 21-29 and 22-29, obtained from cyanogen bromide cleavage of the $S$ protein, were isolated in a ratio of 1:1 (38) or $1: 1.5$ (58) were taken to indicate that the initial attack of subtilisin BPN' $^{\prime}$ occured at the $\mathrm{Ser}_{21}{ }^{-}$ $\mathrm{Ser}_{22}$ bond. The further degradation of the isolated S-peptide (1-20) by subtilisin BPN' (149) involved hydrolysis of the $\mathrm{Ala}_{19}-\mathrm{Ala}_{20}$ bond, indicating that the enzyme possesses carboxypeptidase actitivy. However, it should be pointed out that the free amino acids liberated in the experiments mentioned above have not been isolated, and that contamination of the subtilisin $\mathrm{BPN}^{\prime}$ preparations by carbox- ypeptidase has not been ruled out. In two cases $(204,205)$ the hydrolysis of a C-terminal peptide bond by subtilisin type Carlsberg has been observed (see Fig. 4) and in both cases the free amino acid residue has been isolated.

The specificity shown in the limited proteolysis of ribonuclease is probably related to the threedimensional structure of the substrate. The susceptible bond(s) is located in a loop on the enzyme surface, and therefore from steric reasons might be more accessible. Ribonuclease from rat pancreas is not attacked at the bonds discussed above, probably because the loop does not exist due to replacement of $\operatorname{Ser}_{18}$ and $\operatorname{Ser}_{23}$ in the bovine enzyme by prolyl residues (94).

As will be apparent from Fig. 4 and the discussion above, the specificity of the subtilisins towards aromatic side-chains as determined from the hydrolysis of small synthetic substrates is less clearly expressed when peptides and proteins of natural origin are used as substrates. This finding indicates that secondary interactions are important in determining the specificity of these enzymes.

\subsection{Secondary Binding Sites}

\subsubsection{Kinetic Studies}

The first systematic investigation of the size of the active site of subtilisin $\mathrm{BPN}^{\prime}$ was performed by MORIHARA and coworkers (123). From experiments in which a series of peptides of varying chain length and a tyrosyl residue in $P_{1}$ were used as substrates they concluded that the enzyme had at least 6 subsities $\left(S_{1}\right.$ to $S_{4}$ and $S_{1}^{\prime}$, $S_{2}^{\prime}$ ). Some of the results are shown in Table III. Stereospecificity was observed for the subsites $S_{2}, S_{3}, S_{1}^{\prime}$ and $S_{2}^{\prime}$ although the change in rate of hydrolysis was not so pronounced in the last two cases. A large side-chain (Tyr or Phe) in $S_{1}^{\prime}$ inhibited hydrolysis while, on the other hand, such a side-chain in $S_{2}^{\prime}$ enhanced hydrolysis.

In a subsequent study, MORIHARA and coworkers (124) performed a more detailed analysis of the individual subsites. The substrates used were of the type Z-A-Leu- $\mathrm{NH}_{2}$ and Z-GlyLeu- $B$, the leucyl residue always occupying subsite $S_{1}$. The results of the investigations can be summarized as follows:

1) It was confirmed that subsites on the C- 
Table III

Side-chain specificity and stereospecificity of subtilisin BPN' determined by the hydrolysis of various peptides. Data obtained from ref. (123). The arrow indicates the bond to be hydrolyzed.

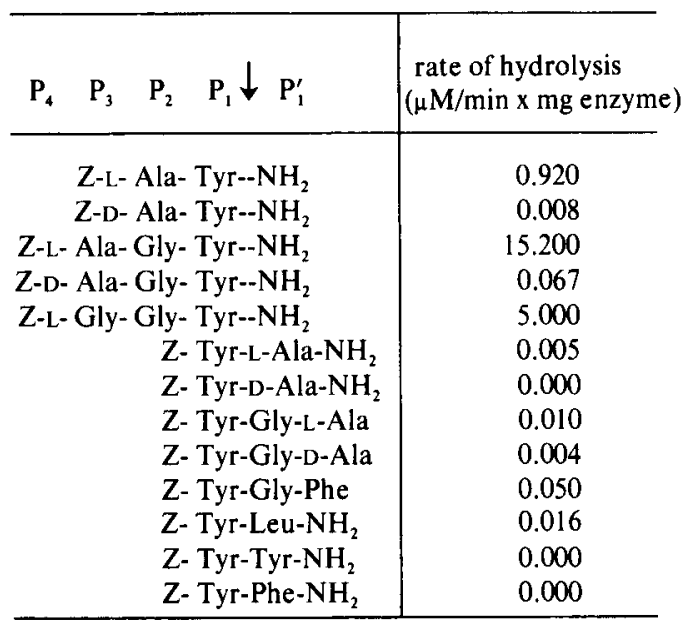

terminal side of the hydrolyzed bond did not extend beyond $\mathrm{S}_{2}^{\prime}$. Only very weak stereospecificity was observed in what would be subsite $S_{3}^{\prime}$ when L-Ala was substituted by D-Ala.

2) On the other hand, a subsite $\mathrm{S}_{4}$ on the Nterminal side of the susceptible bond was recognized by changing D-Ala for L-Ala in the peptide Z-Ala-Gly-Gly-Leu $\mathrm{NH}_{2}$.

3) The importance of $S_{2}$ was demonstrated by exchanging a Gly with an Ala in the peptide ZGly-Gly-Leu $-\mathrm{NH}_{2}$. An increase in the catalytic efficiency $\left(k_{c a t} / K_{M}\right)$ of 14 was observed. However, when Gly was replaced by Tyr a decrease in the rate of hydrolysis was observed as demonstrated by the peptides Z-Tyr-Leu $\pm \mathrm{NH}_{2}$ and Z-Gly-Leu $\mathrm{NH}_{2}$. A subsite, $\mathrm{S}_{2}$, apparently exists which is very restrictive with respect to the size of the amino acid side-chain that binds to it. Thus, Gly is too small to make ideal contacts, while Tyr is too large to be accomodated beneficially. Exchange of a bulky side-chain for Gly in $S_{1}^{\prime}$ has a similar effect.

4) Determination of $k_{\text {cat }}$ and $K_{M}$ for a series of substrates showed that the effects of the secondary binding sites were mainly exerted on the rate of catalysis and to a much smaller extent on the binding. Studies with peptide inhibitors pointed toward the same conclusion. The substrate Z-GlyLeuะGly-Ala was hydro- lyzed in the presence of Z-Gly-Leu-Phe, Z-GlyLeu-Leu, Z-Gly-Leu-Ala and Z-Gly-Leu-DAla. Competitive inhibition was observed and $K_{I}$ was of the same magnitude as $K_{M}$ for the substrate Z-Gly-Leu $\mathrm{NH}_{2}$. Also the hydrolysis of Z-Gly-Leu $\pm \mathrm{NH}_{2}$ in the presence of Z-Gly-DLeu- $\mathrm{NH}_{2}$ showed strong competitive inhibition with a $K_{I}$ identical to the $K_{M}$ of the substrate. Since $K_{\text {I }}$ 's are more reliable measures of binding than are $\mathrm{K}_{\mathrm{M}}$ 's the close correlation between the two values in the cited study suggests that the measured $K_{M}$ 's come close to the true binding constants of the substrates.

The question then arises as to the nature of the effect of the secondary binding sites on the $\mathrm{k}_{\mathrm{cat}}$. Unfortunately the authors do not offer any explanation. It appears to be a common feature of many studies on the secondary binding sites of proteolytic enzymes, that the changes in the rate of hydrolysis observed by changing amino acid side-chains of the substrates, are expressed in $k_{c a t}$ rather than in $K_{M}$. The explanation usually offered is that the binding of substrates at the secondary binding sites leads to subtle conformational changes in the catalytic site of the enzyme resulting in a more efficient attack on the susceptible bond. An experimental verification of this "induced fit " explanation, however, is still lacking.

The subtilisins are inactivated by carbobenzoxyphenylalanine chloromethyl ketone (ZPCK) which reacts specifically with $\mathrm{His}_{64}$ in the catalytic site (see section 2.4). MoriHARA and OKA (125) prepared the chloromethyl ketones of carbobenzoxy-L-alanyl-L-phenylalanine (ZAPCK), and carbobenzoxy-L-alanyl-glycylL-phenylalanine (ZAGPCK), and compared the rate of inactivation of subtilisin $\mathrm{BPN}^{\prime}$ by the three compounds. The relative rates of inactivation were ZPCK:ZAPCK:ZAGPCK = $1: 8: 260$.

As in the earlier studies by MORIHARA and coworkers, it was demonstrated that elongation of the peptide-chain resulted in improved reactivity with the enzyme. The sharp rise in reactivity in going from ZAPCK to ZAGPCK could demonstrate the inability of $S_{3}$ to accomodate a large side chain (as shown with $\mathrm{S}_{2}$ ) or the preference of $S_{4}$ for a hydrophobic sidechain. 
Table IV

The rate of inactivation of subtilisin type Carlsberg and subtilisin BPN' by chloromethylketones of N-terminal blocked peptides of increasing chain-length.

\begin{tabular}{|c|c|c|c|}
\hline \multirow[b]{2}{*}{ Enzyme } & \multicolumn{3}{|c|}{ Rate of inactivation $\left(\mathrm{k} \times 10^{-5} \mathrm{sec}^{-1}\right)$} \\
\hline & ZPCK & ZAPCK & ZAGPCK \\
\hline $\left.\mathrm{BPN}^{\prime 1}\right)$ & 3.5 & 9.3 & 311 \\
\hline $\mathrm{BPN}^{\prime 2}$ ) & 1.0 & 7.9 & 260 \\
\hline Novo ${ }^{1}$ ) & 4.6 & 11.9 & 367 \\
\hline Carlsberg $\left.{ }^{1}\right)$ & 9.2 & 26.8 & 284 \\
\hline
\end{tabular}

1) ref. (126). $\left.\quad{ }^{2}\right)$ ref. (125).

MORIHARA et al. (126) have also investigated the rate of inactivation of subtilisin type Carlsberg with the same chloromethyl ketone derivatives. Qualitatively the results agreed with those found for subtilisin $\mathrm{BPN}^{\prime}$, namely that the rate of inactivation increased with increasing chain-length. Quantitatively, the rate of inactivation was faster with the Carlsberg enzyme when ZPCK or ZAPCK were used as inactivators (Table IV). In the case of the largest inhibitor ZAGPK the rate of inactivation was probably the same (considering the spreading of the results noted for the 3 experiments performed with subtilisin $\mathrm{BPN}^{\prime}$ ).

SHAW and coworkers (31) have shown that the chloromethyl ketone of the tripeptide Phe-Ala-
Lys (PALCK) inactivates subtilisin BPN'. This finding is interesting since the positively charged lysyl residue in $P_{1}$ will not accomodate easily in the hydrophobic subsite $S_{1}$ which bind the phenylalanyl residues in the chloromethylketones used by MORIHARA. It is possible that a different binding mode exists for PALCK. Future kinetic and X-ray crystallographic studies might clarify this point.

In the paper by MORIHARA and coworkers (126) a comparison was made between subtilisin BPN' and subtilisin type Carlsberg in their action on a series of amide substrates with either Tyr or Leu in position $P_{1}$. Some of the data are given in Table V. Prolongation of the peptidechain led also to an increase in the rate of

Table $V^{1}$ )

Rate of hydrolysis of amides of N-terminal blocked peptides of increasing chain-length by subtilisin type Carlsberg and subtilisin BPN'.

Rate of hydrolysis

( $\mu \mathrm{M}$ substrate/mg enzyme $\mathrm{x} \mathrm{min}$ )

\begin{tabular}{r|c|c|c}
\hline$P_{3} P_{4} P_{3} P_{2} \quad P_{1} \quad P_{1}^{\prime}$ & BPN $\left.^{2}\right)$ & Novo & Carlsberg \\
\hline & & & 0.04 \\
Z-Tyr- $\mathrm{NH}_{2}$ & 0.03 & 0.10 & 0.09 \\
Z-Gly- Tyr- $\mathrm{NH}_{2}$ & 0.10 & 0.95 & 1.84 \\
Z-Ala- Tyr- $\mathrm{NH}_{2}$ & 0.92 & 0.01 & 0.04 \\
Z-Tyr- Tyr- $\mathrm{NH}_{2}$ & 0.02 & 2.26 & 1.52 \\
Z-Gly-Gly-Tyr- $\mathrm{NH}_{2}$ & 2.50 & 7.22 & 4.78 \\
Z-Ala-Gly-Tyr- $\mathrm{NH}_{2}$ & 7.05 & 7.70 & 14.68 \\
\hline
\end{tabular}

\footnotetext{
1) From Ref. 126.

2) Product of Nargase Company Japan.

3) Product of Novo, Copenhagen, identical to subtilisin BPN‘.
} 
hydrolysis, when L-amino acids were used in the case of subtilisin type Carlsberg.

Some quantitative differences between the action of the two enzymes are noted. Specially interesting is the large increase in the rate of hydrolysis (about 10 times) by subtilisin type

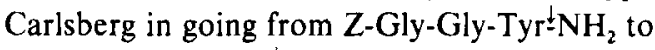

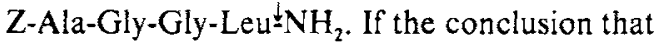
subsite $S_{1}$ accomodates Tyr better than Leu is also correct for subtilisin type Carlsberg, then the observed effect can be located at $\mathrm{S}_{4}$ or $\mathrm{S}_{5}$. No similar pronounced increase is observed with subtilisin $\mathrm{BPN}^{\prime}$ for the substrates mentioned above. This is also seen by comparing

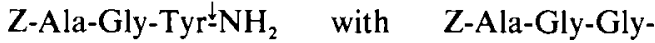
Leu ${ }^{\mathfrak{l}} \mathrm{NH}_{2}$, the hydrolysis by subtilisin $B \mathrm{PN}^{\prime}$ is the same, while an increase of threefold is noted with subtilisin type Carisberg.

It was also shown, that $S_{1}^{\prime}$ and $S_{2}^{\prime}$ in subtilisin type Carlsberg were stereospecific, but not group specific.

\subsubsection{X-ray Crystallographic Studies}

The inhibition studies of subtilisin BPN' with chloromethyl ketone derivatives of various peptides were extended by KRAUT and coworkers to also include X-ray crystallographic analysis $(98,159)$. The chloromethyl ketones of four peptides were used: Z-Ala-Phe, ZAla-Gly-Phe, Z-Gly-Gly-Phe, and Ac-Ala-Gly-

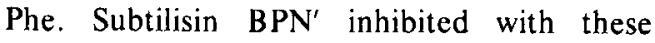
reagents was crystallized and subjected to $\mathrm{X}$ ray crystallographic analysis. As expected, all four inhibitors were found to react with His-64 and they were bound to the enzyme in such a way as to form an antiparallel $\beta$-sheet type of hydrogen bonds with the back-bone part of residues nr 125-127 (see Fig. 5).

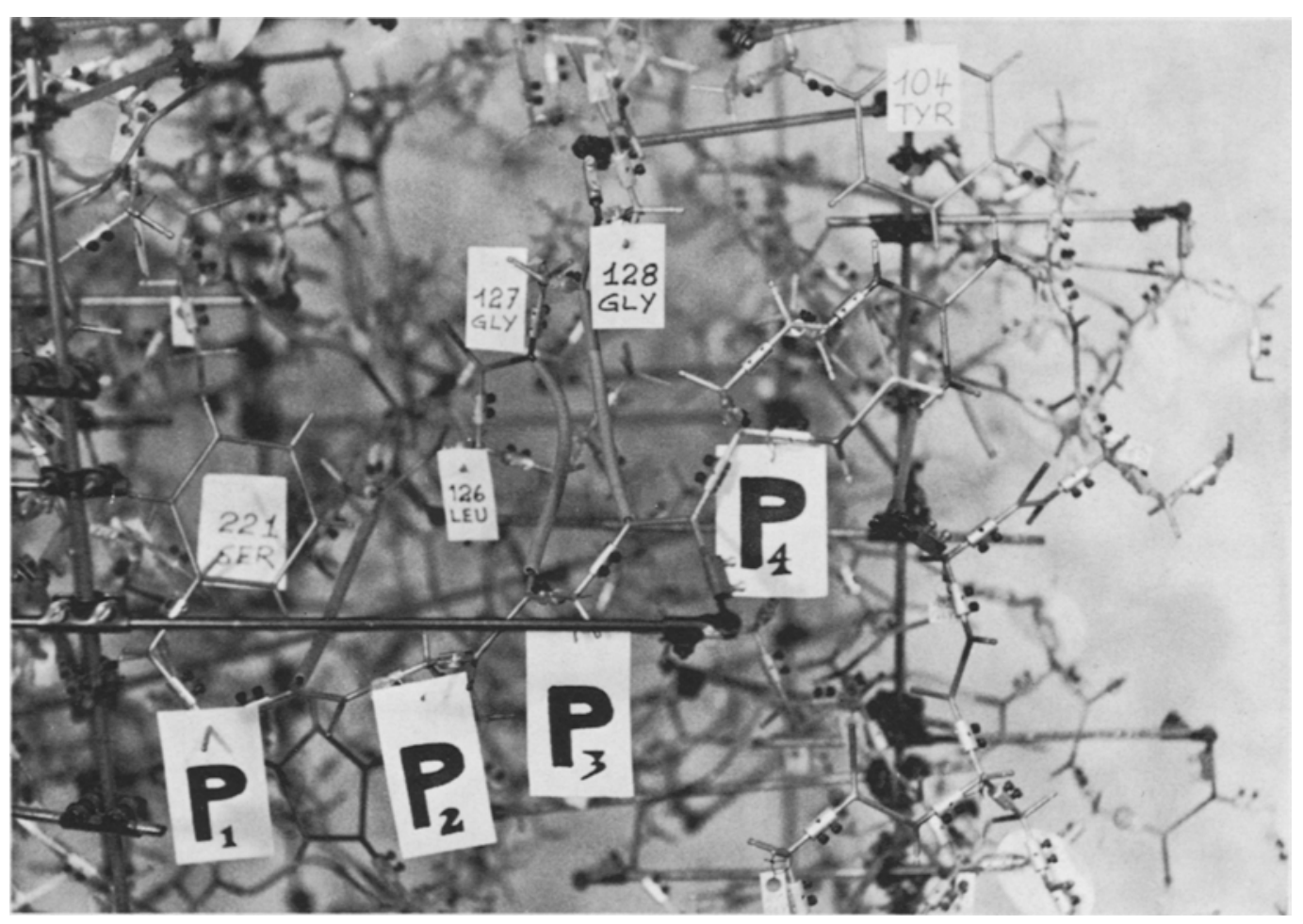

Figure 5. The active site region $S_{1}-S_{4}$ in subtilisin $B P N^{\prime}$. A hypothetical peptide Phe-Ala-Gly-Arg $\left(P_{1}-P_{4}\right)$ is fitted according to the binding mode proposed by KRAUT and coworkers. The benzene ring of Phe is placed in the hydrophobic groove of $S_{1}$ above $\operatorname{Ser}_{221}$. The methyl side-chain of Ala (hidden behind the $P_{2}$ label) is pointing towards the enzyme surface. If an amino acid residue with a side-chain was located in $S_{3}$ the side-chain would have pointed towards the viewer. The side-chain of Arg in subsite $S_{4}$ is extending towards $\mathrm{Tyr}_{104}$, under which phenol ring the guanidine moiety of Arg is seen. Three pieces of rubber tubing indicate the hydrogen bonds, mentioned in the text, between enzyme and substrate. 
Subsite $\mathrm{S}_{1}$, which in all cases accomodated a Phe side-chain, was shown to be a hydrophobic crevice. This is in agreement with the studies already mentioned which pointed toward a preference of subtilisin $\mathrm{BPN}^{\prime}$ for an aromatic side-chain (or leucine) in $\mathrm{P}_{1}$. Model building showed that it was not difficult to place a leucine side-chain in the crevice.

The interaction of subsite $S_{2}$ with $P_{2}$ is much less specific. Van der Waal's contacts are observed between the $\alpha$-carbon of $\mathrm{P}_{2}$ and the $\alpha$ carbon of Leu $_{126}$. With Ala in $\mathrm{P}_{2}$ the methyl side-chain makes van der Waal's contacts with $\mathrm{Leu}_{96}$ and $\mathrm{His}_{64}$. A larger side-chain than Ala (f.ex. a Tyr) would interfere with the proper alignment of the susceptible bond, while a Gly in $\mathrm{P}_{2}$ allows greater freedom of motion and therefore retards proper alignment. These findings are in agreement with the kinetic data of Morithara et al. (124) discussed in the preceding section.

According to RoBERTUS et al (159) subsite $S_{3}$ is more restrictive than $S_{2}$ in its interaction with the peptides in the sense that hydrogen bonds are formed, one between $\mathrm{NH}$ of $\mathrm{P}_{3}$ and $\mathrm{CO}$ of Gly $_{127}$ (Fig. 5). On the other hand, the sidechain of $\mathrm{P}_{3}$ points out into the surrounding medium and its subsite should therefore be able to accomodate even large side-chains. This assumption is verified by kinetic data (124) since f.ex. Z-Phe-Gly-Leu $\_\mathrm{NH}_{2}$ is a better substrate than Z-Gly-Gly-Leu ${ }^{\downarrow} \mathrm{NH}_{2}\left(\mathrm{k}_{\text {cat }} / \mathrm{K}_{\mathrm{M}}\right.$ increases 6.5 times). Because the hydrogen bonds between substrate and enzyme must be identical for the two amino acids in $\mathrm{P}_{3}$, the increase in catalytic efficiency most likely stems from van der Waal's contacts between the benzene ring and the enzyme surface as suggested by KRAUT and coworkers in the case of the Z-Ala-Phe derivative where the $Z$ moiety occupies $S_{3}$. Finally, subsite $S_{4}$ was recognized as another, very deep, crevice the deepest part of which was sealed off by $\mathrm{Tyr}_{104}$. With a $\mathrm{Z}$ moiety in $\mathrm{P}_{4}$ a movement of the phenol-ring of $\mathrm{Tyr}_{104}$ was observed, but the new spatial orientation was difficult to locate precisely. It was possible to orient the tyrosine ring parallel to the benzene ring of $Z$ at a distance of $3.5 \AA$, which placed it in van der Waal's contact with this group. The inhibitor Z-Ala-Phe (160) was shown to bind in reverse of the chloromethyl ketones, that is, with Phe in subsite $S_{4}$. In this case the new location of $\mathrm{Tyr}_{104}$ was clearly defined. It was shown that a hydrogen bond was formed between the hydroxyl group of $\mathrm{Tyr}_{104}$ and the carboxyl group of Phe. If the N-terminal blocking group was an acetyl group rather than a carbobenzoxy group, as in Ac-Ala-Gly-Phechloromethyl ketone, no movement of $\mathrm{Tyr}_{104}$ was observed. From these observations KRAUT and coworkers concluded that subsite $\mathrm{S}_{4}$ has a very strong affinity for a Phe side-chain and that this site "plays an important role in determining the cleavage point for polypeptide substrates" (160).

In the work just referred to (160), the La Jolla group extended their work also to include the following peptides: Z-Gly-Gly-Tyr, Z-Gly-GlyLeu and Z-Gly-Gly-Arg. These peptides, called "virtual substrates", are splits products of good substrates and are assumed to bind identically with the substrates from which they are derived. Z-Gly-Gly-Tyr was bound in the same manner as was observed with the chloromethyl ketone derivatives, except for the lack of a hydrogen bond in the catalytic site, a feature which probably is of importance for the catalytic mechanism. As discussed in section 3.2. this bond is probably first formed in the transition state.

The binding of Z-Gly-Gly-Leu was less clearly defined, but the data were still of good enough quality to show two binding modes of about equal strength for $P_{1}$. One was identical to that observed for Z-Gly-Gly-Tyr, while in the other Leu was twisted down in the vicinity of $\mathrm{His}_{64}$, that is, away from the hydrophobic crevice of $S_{1}$. Furthermore, considerable binding was observed in the vicinity of a surface depression formed by the side-chains of $\mathrm{Tyr}_{217}, \mathrm{Met}_{222}$ and $\mathrm{His}_{67}$, and also at another surface depression under Phe $_{189}$. These two areas were termed "sticky spots" and probably represent areas that bind substrates less firmly than the area described in detail above.

The data for binding of Z-Gly-Gly-Arg were the most difficult to interpret. One binding mode identical to that of Z-Gly-Gly-Tyr and one identical to the second binding mode of Z-GlyGly-Leu were observed, but the second binding 
mode was dominant. Binding at the wsticky spots" was indicated as well.

These results agree with the data from kinetic studies. Tyr in $P_{1}$ makes the substrate a good one and therefore clear-cut X-ray data could be obtained with Z-Gly-Gly-Tyr. On the other hand the Leu and especially the Arg peptides are less satisfactory substrates in agreement with the more diffuse $\mathrm{X}$-ray data containing additional binding modes. It also appears reasonable that the positively charged Arg would have difficulties in accomodating to the same hydrophobic crevice as Tyr.

Subsites $S_{1}^{\prime}$ and $S_{2}^{\prime}$ which have been demonstrated kinetically have not been located by direct X-ray diffraction studies, but model building shows that only a very limited number of stereochemical convincing possibilities exists for these sites. The "sticky spots" appear to be likely candidates. Thus the region around $\mathrm{Tyr}_{217}$ is tentatively identified as subsite $S_{1}^{\prime}$; $\mathbf{S}_{2}^{\prime}$ is probably located below Phe $_{189}$. Model building also showed that the existence of a $S_{3}^{\prime}$ subsite appeared quite unlikely.

In a recent report (168) preliminary X-ray data have been published on the crystalline complex between subtilisin BPN' and a specific inhibitor isolated from Streptomyces albogriseolus S-3253. It will be very interesting to see how big an area of the subtilisin molecule is involved in the binding of this large inhibitor, especially the part corresponding to $S_{1}^{\prime}$ and $S_{2}^{\prime}$ for which convincing data still are lacking.

\subsubsection{Productive Binding Modes}

The $\mathrm{X}$-ray crystallographic analyses discussed in some detail above provide convincing evidence for the existence of at least one way of binding peptides to subtilisin $\mathrm{BPN}^{\prime}$ that leads to hydrolysis. However, is the productive binding mode demonstrated by KRAUT and coworkers the only possible one? Additional binding modes were shown for Z-Gly-Gly-Leu and ZGly-Gly-Arg, but these were considered to be non-productive. WRIGHT et al. (218) demonstrated that $\mathrm{N}$-benzoyl-L-arginine (Bz-Arg) was bound in a way different from that of synthetic peptides, but they were unable to conclude whether it represented productive or non-productive binding.
Recently WRIGHT (217) has argued that two binding sites exist near the catalytic site which are both productive. These binding sites were termed $\mathbf{A}$ and $\mathbf{B}$. Binding site $\mathbf{A}$ is identical to subsite $S_{1}$ and functions when large peptide substrates are hydrolyzed, because these substrates are able to form the "pleated sheet type of interaction with subsites $S_{1}$ to $S_{4}$. Binding site B seems to bear close resemblance to subsite $S_{1}^{\prime}$ and is operative in binding small aromatic inhibitors and certain ester substrates. The site has been observed in crystals of PMSF inhibited subtilisin $\mathrm{BPN}^{\prime}(216)$, pipsyl- and transcinnamoyl subtilisin $\mathrm{BPN}^{\prime}$, and Bz-Arg inhibited subtilisin BPN' $^{\prime}$. The reaction with PMSF must be considered productive in the sense that the catalytic seryl residue is sulphonylated, although desulphonylation does not take place under conditions normal for the enzymatic function of the enzyme. However, deacylation of the intermediate takes place in the reaction between $\mathrm{N}$-trans-cinnamoyl imidazole and the subtilisins, clearly demonstrating that binding site $\mathrm{B}$ is productive. Thus $\mathrm{X}$-ray diffraction data seem to provide evidence for more than one productive binding mode in subtilisin $\mathrm{BPN}^{\prime}$, and kinetic evidences also point towards the same conclusion.

Inhibition studies carried out by GLAZER (53) on both subtilisins showed that the hydrolysis of $\mathrm{N}$-acetyl tyrosine ethyl ester (ATEE) was inhibited competitively by certain aromatic compounds (phenol, indole, hydrocinnamate, and indolepropionate), while non-competitive inhibition was observed when the substrate was N-benzoyl-L-arginine ethyl ester (BAEE). These findings suggested different modes of productive binding for the two substrates, and the broad specificity of the subtilisins could probably in part be explained in this way.

WRIGHT (217) also conducted kinetic inhibition studies in conjunction with the $\mathrm{X}$-ray analysis mentioned above. Competitive inhibition by $\mathrm{Bz}$ Arg of the hydrolysis of TCI by subtilisin BPN' was observed suggesting that both compounds bind to the same site (site B). Competitive inhibition was also observed with indole, while Bz-Gly inhibited the reaction noncompetitively. Since N-acetyl-L-arginine had no effect on the hydrolysis of TCI, it was suggested that the 
benzoyl moiety was mainly responsible for the binding of Bz-Arg. This inhibitor is apparently large enough to block binding site $\mathrm{B}$, while $\mathrm{Bz}$ Gly only distorts the substrate binding at this site.

Finally, kinetic evidence for the existence of more than one productive binding mode in the subtilisins has been obtained by BossHARD (21). With subtilisin $B P N^{\prime}$ it was shown that the hydrolysis of Ala-Ala-Phe-OMe and Ac-AlaAla-Phe-Ala was inhibited competitively by ZAla-Ala-Phe, while non-competitive inhibition was observed with Bz-Arg. On the other hand the hydrolysis of $\mathrm{TCI}$ was inhibited competitively by $\mathrm{Bz}$-Arg and non-competitively by Z-Ala-Ala-Phe. All these findings agree with the binding modes proposed already. Thus AcAla-Ala-Phe, Z-Ala-Ala- Phe-OMe, and Z-AlaAla-Phe all bind at site $\mathrm{A}$, while $\mathrm{TCI}$ and $\mathrm{Bz}$ Arg bind at site B. From studies on the binding of D-amino acid residues to the subtilisins the same author (23) concluded that "no uniform binding mode exists".

The hydrolysis of BAEE was inhibited noncompetitively by both Z-Ala-Ala-Phe and BZArg (21). These results were explained by binding of BAEE at both sites, but not simultaneously. Since Bz-Arg binds at site B only, non-competitive inhibition is observed for BAEE bound to site A, while Z-Ala-Ala-Phe bound in site $\mathrm{A}$ inhibits BAEE bound in site $\mathrm{B}$. However a third binding mode for BAEE could not be excluded.

Identical results were obtained with subtilisin type Carlsberg except for inhibition by Bz-Arg, which inhibited all substrates competitively. It was suggested that in the Carlsberg enzyme both binding sites were covered partly by this inhibitor. Inspection of the three-dimensional model of subtilisin type Carlsberg shows that while no apparent differences exist between this enzyme and subtilisin BPN' with respect to Subsites $S_{1}$ and $S_{4}$, the binding areas corresponding to $S_{1}^{\prime}$ and $S_{2}^{\prime}$ are not identical. This observation suggests that the differences in enzymatic behavior between the subtilisins are mainly due to differences in binding of $\mathrm{P}_{1}^{\prime}$ and $\mathrm{P}_{2}^{\prime}$. It is therefore quite reasonable that inhibition of subtilisin type Carlsberg by Bz-Arg is different from that of the $B P N^{\prime}$ enzyme. In a recent publication (22) BOSSHARD and BERGER argue that the $S_{1}$ binding site of subtilisin type Carlsberg is much narrower than that of subtilisin $\mathrm{BPN}^{\prime}$. This conclusion rests on the finding that the inhibitor Z-Ala-Ala-CysS-diphenylmethyl binds strongly to subtilisin $\mathrm{BPN}^{\prime}$ enzy$\operatorname{me}\left(\overline{\mathrm{K}}_{\mathrm{I}}=71,000 \mathrm{M}^{-1}\right)$ while subtilisin type Carlsberg is inhibited to a much smaller extent $\left(\overline{\mathbf{K}}_{\mathbf{I}}=\right.$ $13,000 \mathrm{M}^{-1}$ ). According to BosshaRD and BERGER the weaker binding is due to a narrower $S_{1}$ which cannot accomodate the big diphenylmethyl group. However, the present author suggests that the diphenylmethyl group, because of its size, can bind to subsite $S_{1}^{\prime}$ equally well and that this subsite is more suitable in subtilisin $\mathrm{BPN}^{\prime}$. In this connection it is interesting to note that 2-nitro-4-carboxyphenyl-N,N-diphenylcarbamate was hydrolyzed three times faster by subtilisin type Carlsberg than BPN $^{\prime}$ (see section 3.3.1).

The qualitative results of the inhibition studies discussed above are summarized in Table VI.

The unexpected finding that the bond between $\mathrm{Leu}_{15}$ and $\mathrm{Tyr}_{16}$ in the B-chain of oxidized insulin is hydrolyzed much faster than any other bond (85) can now be rationalized in terms of the binding modes proposed by KRAUT and coworkers. Although $\mathrm{Tyr}_{16}$ a priori would be expected to bind in $S_{1}$, Leu 15 is still a reasonably good candidate. $\mathrm{Ala}_{14}$ is accomodated perfectly in $S_{2}$. Furthermore, subsite, $S_{3}$ is not side chainspecific and should easily accomodate $\mathrm{Glu}_{13}$. Finally, $\mathrm{Val}_{12}$ is expected to bind at the hydrophobic $S_{4}$. If we turn to the C-terminal side of the susceptible bond, $\mathrm{Tyr}_{16}$ and $\mathrm{Leu}_{17}$ appear to be excellent candidates for occupying the "sticky" subsite $S_{1}^{\prime}$ and $S_{2}^{\prime}$. Thus, the very rapid hydrolysis of the $\operatorname{Leu}_{15}-\mathrm{Tyr}_{16}$ bond in the B-chain of oxidized insulin can be explained by the excellent fit of the six residues around this bond with the subsites on the enzymes.

\subsubsection{Secondary Binding Sites Affected by Chemi- cal Modifications}

In the two preceding sections, the elucidation of secondary binding sites by means of $\mathrm{X}$-ray diffraction studies of bound inhibitors or virtual substrates and by means of kinetic studies using various inhibitors has been discussed. We now turn to a third way in which information can be 
Table VI

Types of inhibition observed when substrates are hydrolyzed by subtilisin $\mathrm{BPN}^{\prime}$ and subtilisin type Carlsberg in the presence of various inhibitors.

\begin{tabular}{l|c|c|c|c|c|c}
\hline \multirow{2}{*}{ Inhibitor } & \multicolumn{7}{c}{ Substrate } \\
\hline (subtilisin BPN') & TCI & BAEE & ATEE & AAPOMe & AcAAPA & Ref. \\
\cline { 2 - 7 } Bz-Arg & C & NC & - & NC & NC & 217,21 \\
Phenol, Indole & C & NC & C & - & - & 53,217 \\
Bz-Gly & NC & - & - & - & - & 217 \\
Z-Ala-Ala-Phe & NC & NC & - & $\mathrm{C}$ & $\mathrm{C}$ & 21 \\
(subtilisin Carlsberg) & C & - & C & C & - & 21 \\
Bz-Arg & - & NC & C & - & - & 53 \\
Phenol,Indole & NC & NC & C & C & - & 21 \\
Z-Ala-Ala-Phe & &
\end{tabular}

$\mathrm{C}=$ competitive inhibition, $\mathrm{NC}=$ non-competitive inhibition

obtained about the secondary binding sites, namely chemical modifications of the enzyme. However, those reactions which have to do with modification of $\mathrm{Ser}_{221}$ and $\mathrm{His}_{64}$ will not be discussed since these groups are part of the catalytic site.

Nitration or iodination of tyrosyl residues with tetranitromethane (TNM) led to drastic changes in the hydrolysis of certain substrates by both subtilisin type Carlsberg and subtilsin BPN' $(84$, 188). It was found that the hydrolysis of the small synthetic ester substrates BTEE (84) and TAME (188) is affected only slightly by the modification of tyrosyl residues in both enzymes. These results are in agreement with the X-ray diffraction studies which showed that no tyrosyl residues are found at the catalytic site. The substrate BTEE will most likely bind in such a way that the tyrosyl side-chain occupy subsite $S_{1}$ (or binding mode $A$ ). The ethyl group in $S_{1}^{\prime}$ probably does not extent far enough to get into contact with $\mathrm{Tyr}_{21}$ in subtilisin BPN'. Position 217 is not occupied by a Tyr in subtilisin type Carlsberg. $\mathrm{Tyr}_{209}$, which might be located in the active site region of this enzyme, is even further removed from the catalytic site than is $\mathrm{Tyr}_{217}$ in subtilisin $\mathrm{BPN}^{\prime}$.

Table VII shows the catalytic parameters for the hydrolysis of TAME with subtilisin type Carlsberg and subtilisin BPN' and their nitrated and iodinated derivatives. Whether TAME is bound productively in mode $A$ or $B$, its hydrolysis by the subtilisins is not influenced to any great extent when these enzymes are nitrated or iodinated.

The picture becomes quite different when large

Table VII

Determination of the Michaëlis-Menten constants $K_{m}$ and $V_{\max }$ for toluenesulfonyl-targinine methyl ester with native and modified subtilisins.

\begin{tabular}{l|c|c}
\hline & $K_{m}(M)$ & $\left.V_{\max }{ }^{1}\right)$ \\
\hline Native subtilisin type Carlsberg & $3.7 \times 10^{-2}$ & 1540 \\
Nitrated subtilisin type Carlsberg & $2.8 \times 10^{-2}$ & 1335 \\
lodinated subtilisin type Carlsberg & $2.0 \times 10^{-2}$ & 1390 \\
Native subtilisin BPN' & $3.0 \times 10^{-2}$ & 100 \\
Nitrated subtilisin BPN' & $2.4 \times 10^{-2}$ & 122 \\
\hline
\end{tabular}

1) Expressed as $\mu$ eqv. $\mathrm{H}^{+}$liberated per min per $\mathrm{mg}$ enzyme per $\mathrm{ml}$ in a $10 \mathrm{ml}$ incubation mixture. The experiments were performed at $\mathrm{pH} 8.0$ and $30^{\circ} \mathrm{C}$. 


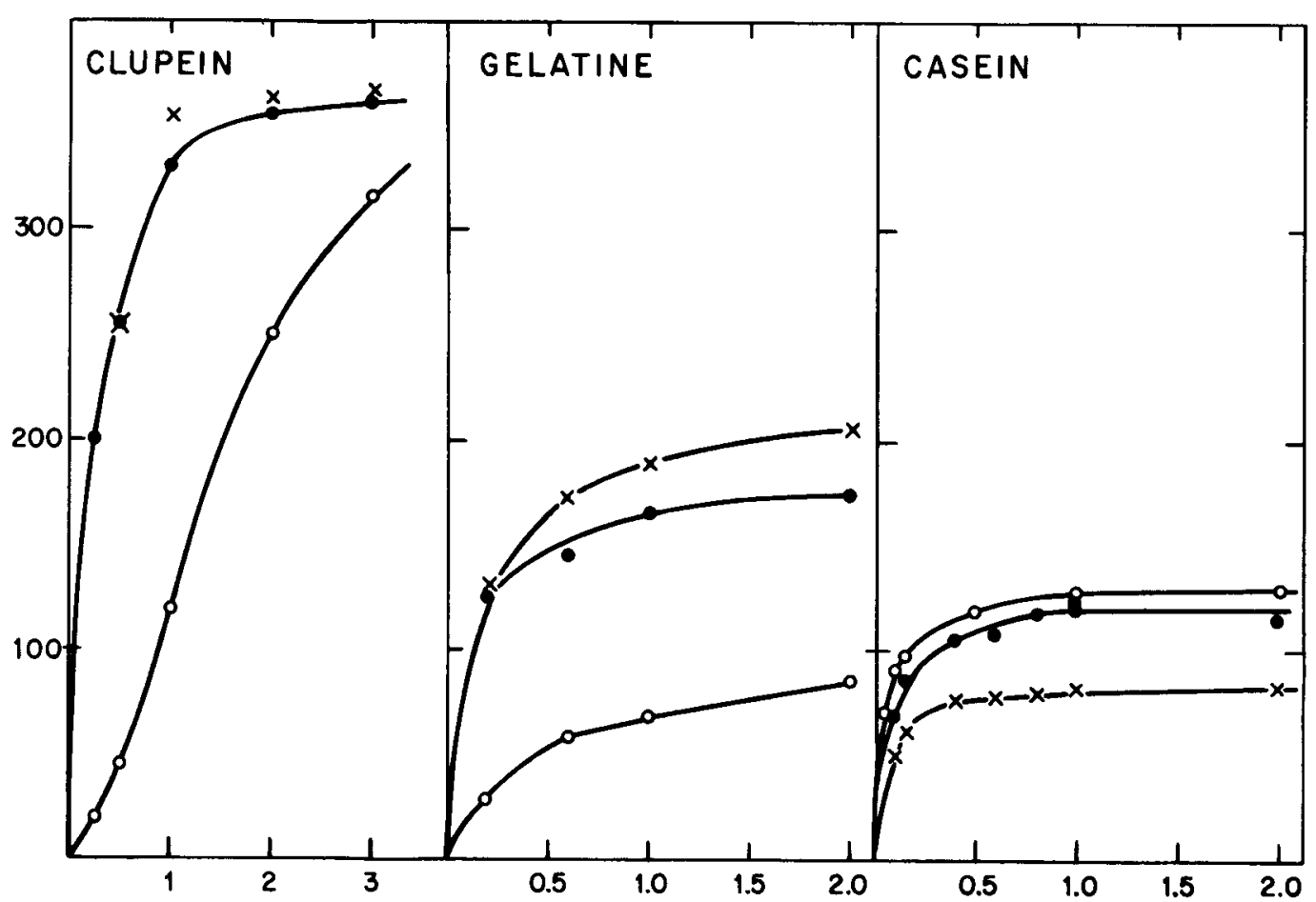

Figure 6. Hydrolysis of clupein sulphate, gelatine and casein by subtilisin type Carlsberg and its nitrated and iodinated derivatives. $-\mathrm{O}-$ unmodified enzyme, -- nitrated enzyme, $-x-$ iodinated enzyme. Abscissa: substrate concentration in per cent. Ordinate: rate of hydrolysis expressed as $\mu$ eq $\mathrm{H}^{+}$liberated per min per mg enzyme per $\mathrm{ml}$ in a $10 \mathrm{ml}$ incubation mixture. $\mathrm{pH}=8.0, \mathrm{t}=30^{\circ} \mathrm{C}$.

peptide substrates are hydrolyzed by iodinated or nitrated subtilisins. Thus a sevenfold increase was observed in the hydrolysis of a $0.5 \%$ clupein sulphate solution after nitration or iodination of subtilisin type Carlsberg.

A more detailed kinetic analysis showed that the enhancement of catalysis was due to a lowering of $\mathrm{K}_{\mathrm{M}}$ (app) rather than an increase in $V_{\max }$, which was found to be nearly constant (see Fig. 6). These results are quite different from those of MORIHARA et al. (124) obtained by varying the individual amino acid residues in synthetic peptide substrates. They found that $\mathrm{k}_{\text {cat }}$ changed and $\mathrm{K}_{\mathrm{M}}$ remained almost constant.

A similar, but not so drastic effect was observed when gelatine was the substrate (188). In this case an increase in $V_{\max }$ was found as well as a decrease in $K_{M}$ (app) (Fig. 6). Finally, the hydrolysis of casein by subtilisin type Carlsberg was unaffected by nitration of the enzyme while iodination caused a decrease in $V_{\text {max }}$ (Fig. 6).
The hydrolysis of clupein by subtilisin $\mathrm{BPN}^{\prime}$ was also influenced by nitration or iodination. $\mathrm{K}_{\mathrm{M}}$ (app) was decreased as was the case with the Carlsberg enzyme but, in contrast $V_{\max }$ was decreased to about half of the value obtained with the unmodified enzyme (Fig. 7). Also the rate of hydrolysis of gelatine by the modified subtilisin BPN' $^{\prime}$ was different since a minor decrease, instead of an increase, was observed upon iodination or nitration while the rate of hydrolysis of casein was totally unaffected (Fig. 7).

How do we interpret these results? First of all, it was clear that the chemical modifications of tyrosyl groups were the cause of the changes in enzymatic properties, since reduction of the derivative with sodium dithionite, which changed the 3-nitrotyrosyl residues into 3-aminotyrosyl residues, resulted in catalytic properties identical to those of the unmodified enzymes (188). The $\mathrm{pK}_{\mathrm{a}}$ of tyrosine is about 10 while the $\mathrm{pK}_{\mathrm{a}}$ of 3-nitrotyrosine is about 7 (184). Since 
all the experiments discussed above were performed at $\mathrm{pH} \mathrm{8.0,} \mathrm{the} \mathrm{tyrosyl} \mathrm{residues}$ in the modified enzymes carried a negative charge while they were neutral in the unmodified enzyme. It was therefore suggested that introduction of a negative charge at one or more locations on the enzyme surface created new binding sites for large peptides or improved already existing sites.

All the experiments discussed above were performed with enzymes having a high degree of modification (5 to 7 residues nitrated or iodinated in subtilisin type Carlsberg, 4 residues modified in subtilisin $\mathrm{BPN}^{\prime}$ ).

In order to determine if modification of all these groups was necessary for the maximal change in activities, subtilisin type Carlsberg was nitrated by addition of TNM in small increments and the corresponding activity towards $0.5 \%$ clupein determined. It was shown that the maximal change in rate of hydrolysis was obtained when between one and two tyrosyl residues had been modified. Thus the observed effect was probably local and not due to a general change in the charge pattern.

When the tentative three-dimensional model of subtilisin type Carlsberg was built at the Carlsberg Laboratory, it appeared that there were two candidates for the specific nitration or iodination: $\mathrm{Tyr}_{209}$, already mentioned in connection with hydrolysis of ester substrates, and $\mathrm{Tyr}_{104}$ which was identical in the two subtilisins. $\mathrm{Tyr}_{104}$ is most likely the residue which, when modified, gives rise to the changes in enzymatic properties of subtilisin type Carlsberg. First, $\mathrm{Tyr}_{104}$ constitutes part of the important subsite $\mathrm{S}_{4}$ identified by KRAUT and coworkers. Second, $\mathrm{Tyr}_{104}$ is the first residue to be nitrated (111) or iodinated (211) in subtilisin $\mathrm{BPN}^{\prime}$. Thirdly, reaction of subtilisin type Carlsberg with a tenfold excess of TNM followed by tryptic digestion of the derivative showed that

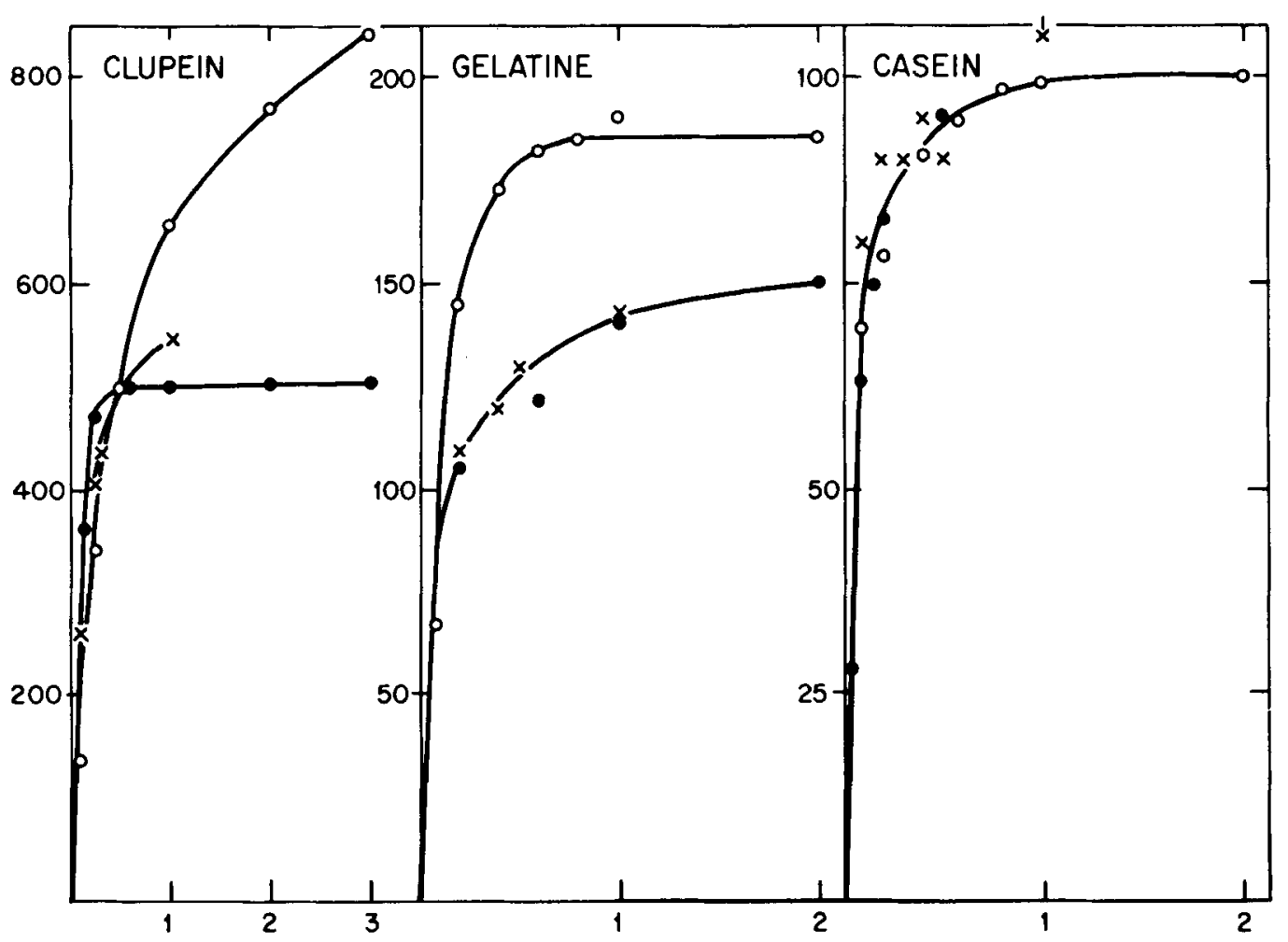

Figure 7. Hydrolysis of clupein sulphate, gelatine and casein by subtilisin BPN' and its nitrated and iodinated derivatives. - - - unmodified enzyme, - - nitrated enzyme, $-\times$ - iodinated enzyme. Abscissa: substrate concentration in per cent. Ordinate: rate of hydrolysis expressed as $\mu \mathrm{eq} \mathrm{H}^{+}$per min per $\mathrm{mg}$ enzyme per $\mathrm{ml}$ in a 10 $\mathrm{ml}$ incubation mixture. Note the difference in scale for the three substrates. $\mathrm{pH}=8.0, \mathrm{t}=30^{\circ} \mathrm{C}$. 
in this enzyme $\mathrm{Tyr}_{104}$ also reacts faster than any of the other tyrosyl residues (190).

Therefore, it is quite safe to assume that nitration or iodination of $\mathrm{Tyr}_{104}$ is the main cause of the lowering of $\mathrm{K}_{\mathrm{M}}$ (app) for the subtilisin type Carlsberg - clupein complex, although it cannot be excluded that nitration or iodination of $\mathrm{Tyr}_{209}$ might play a role.

It should be pointed out that although $\mathrm{Tyr}_{104}$ was the first residue to become nitrated in subtilisin $\mathrm{BPN}^{\prime}$, modification of additional tyrosyl residues was necessary in order to obtain maximal change in the rate of hydrolysis of clupein (111). It is possible that nitration of both $\mathrm{Tyr}_{104}$ and $\mathrm{Tyr}_{217}$ is needed in this case for maximal "efficiency .

Subsite $\mathrm{S}_{4}$ is specially suited for the accomodation of a phenylalanyl side-chain. However, clupein does not contain any aromatic sidechains which would be likely candidates for association with subsite $S_{1}$ or $S_{4}$. On the other hand, the high percentage of Arg makes it probable that an Arg will try to fit in $S_{4}$. However, such a complex will be energetically unfavorable when the enzyme is unmodified, but introduction of a negative charge at this site by nitration of $\mathrm{Tyr}_{104}$ will facilitate the binding of an arginyl side-chain. It is possible that the combination of an extended hydrophobic chain $\left(-\mathrm{CH}_{2}-\mathrm{CH}_{2}-\mathrm{CH}_{2}-\mathrm{CH}_{2}-\right)$ and a positive charge on the substrate with a hydrophobic crevice plus a negative charge on the enzyme will create binding of reasonable strength.

If we now turn to subtilisin $\mathrm{BPN}^{\prime}$, the same argument should apply since subsites $S_{1}$ and $S_{4}$ seem to be identical to those in subtilisin type Carlsberg. The marked decrease in $\mathrm{K}_{\mathrm{M}}$ (app) is also observed when clupein is hydrolyzed, but $V_{\text {max }}$ decreases concomitantly. The simultaneous decrease in $\mathrm{K}_{\mathrm{M}}$ (app) and $\mathrm{V}_{\max }$ has been interpreted as indicating non-productive binding (47). If this is the case, the observed effect is most likely caused by nitration or iodination of $\mathrm{Tyr}_{217}$ since it is located in the $\mathrm{S}_{1}^{\prime}$ area of the active site and since subtilisin type Carlsberg does not have a tyrosyl residues in position 217.

A $0.5 \%$ solution of clupein sulfate is hydrolyzed about 10 times faster by subtilisin BPN' than by subtilisin type Carlsberg at $\mathrm{pH} 8$ and $30^{\circ}$. On the other hand the opposite was found to be the case (188) with the ester substrate TAME under the same conditions. This apparent paradox may be explained in different ways. It is generally accepted that the rate determining step in ester hydrolysis is deacylation of the enzymesubstrate intermediate. As mentioned in section 3.3.1 deacylation of this intermediate is faster with the Carlsberg enzyme than with the BPN' enzyme. On the other hand acylation is usually the rate determining step in amide hydrolysis. The apparent paradox therefore disappears if acylation is fastest for subtilisin $\mathrm{BPN}^{\prime}$ in the hydrolysis of clupein sulfate. An alternative explanation would be that clupein and TAME are bound differently and therefore are not directly comparable.

Probably, the faster hydrolysis of clupein sulfate by subtilisin BPN' compared to subtilisin type Carlsberg is partly due to the presence of more negative charges near the active site region in this enzyme. Although no aspartyl nor glutamyl residues are included in the subsites $S_{1}$ to $S_{4}$ determined by KRAUT and coworkers, it is very likely that the extented side-chains of arginyl residues can get into contact with such negative residues and add to the binding of the substrate.

The hydrolysis of each of the three components in clupein by the subtilisins was investigated in detail (190a). Two of the components, clupein YII and $Z$ differ only slightly in their primary structure. These polypeptides were hydrolyzed with identical rates by subtilisin BPN'. The investigations was concentrated on the initial stage of hydrolysis and it was shown that two bonds, a Ser-Arg and an Ala-Ser, were hydrolyzed faster than any of the other bonds (see Fig. 8), the Ser-Arg bond being most readily attacked. The same two bonds were hydrolyzed by subtilisin Carlsberg, but at a much slower rate. Again, the quantitative rather than qualitative differences between the two enzymes are emphasized. When clupein $\mathrm{Z}$ was hydrolyzed by nitrated subtilisin Carlsberg the specificity for the bond $\operatorname{Ser}_{22}-\operatorname{Arg}_{23}$ had increased. This finding is compatible with an Arg bond placed in subsite $S_{4}$ when this bond is being hydrolyzed, while a $T h r$ is bound in $S_{4}$ when the bond $\mathrm{Ala}_{8}-\mathrm{Ser}_{9}$ is hydrolyzed (Fig. 8). 


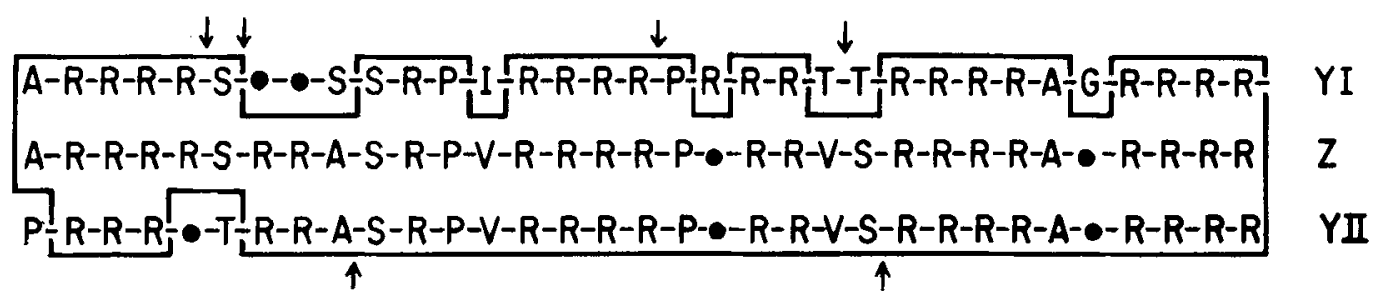

Figure 8. The amino acid sequence of clupein $\mathrm{YI}, \mathrm{Z}$ and $\mathrm{YII}$ arranged to give maximal homology. $\bullet$ indicates presumed deletions. $\uparrow$ principal points of attack in clupein Yll and Z. $\downarrow$ : points of attack in clupein $Y, A=A$ la, $R$ $=\operatorname{Arg}, \mathbf{S}=\operatorname{Ser}, \mathrm{T}=$ Thr, $\mathrm{I}=\mathrm{Ile}, \mathrm{G}=\mathrm{Gly}, \mathrm{V}=$ Val and $\mathrm{P}=$ Pro.

When clupein YI was hydrolyzed by the unmodified subtilisins a complicated pattern of peptides was obtained. Some of the hydrolyzed bonds were identified (Fig. 8) but no major point of attack was detected as with clupein YII and Z. However, when clupein YI was hydrolyzed by nitrated subtilisin Carlsberg the specificity for the bond $\operatorname{Arg}_{5}-\operatorname{Ser}_{6}$ was greatly enhanced. It is not clear why this bond is preferred by the nitrated subtilisin since the hydrolysis of the other bonds which are attacked by unmodified enzyme also places an Arg in subsite $\mathrm{S}_{4}$.

The protamine salmine has been used by GERTLER (48) as substrate for a subtilisin shown by amino acid analysis to be the $\mathrm{BPN}^{\prime}$ type enzyme. Salmine is closely related to clupein, having a high content of arginyl residues. Modification of the enzyme by maleylation of the lysyl residues led to changes in the hydrolysis of salmine sulphate. The maximal rate of hydrolysis was unchanged, but $\mathrm{K}_{M}$ increased five times. This result is surprising since one would expect a decrease in $\mathrm{K}_{\mathrm{M}}$ when the enzyme was made more negative, an effect observed with maleylated elastase in its action on salmine sulfate. It was also found that the hydrolysis of casein and hemoglobin were unaffected. However, elastin was hardly hydrolyzed at all, which was explained by the loss of binding capacity for this substrate. Since no changes were observed in the hydrolysis of the ester substrates ATEE or N-acetyl-Ala-AlaAla-methyl ester it was concluded that the catalytic region was not affected, and the results are also in agreement with the subsite model of KRAUT and coworkers since no lysyl residues are found in the $S_{1}$ to $S_{4}$ region on the enzyme surface.

However, the apparent involvement of lysyl residues in the binding of salmine to subtilisin $\mathrm{BPN}^{\prime}$ is unexpected since lysyl residues are unimportant in the binding of clupein. One explanation would be that salmine binds to subtilisin in a way quite different from that of clupein. A more likely interpretation appears to be that other groups were modified in addition to the lysyl residues. It was shown (48) that maleylation of elastase resulted in a temporary inactivation of the enzyme, but that full activity towards the trialanyl ester substrate was recovered by a short preincubation of the modified enzyme before assay. It is conceivable that more stable derivatives could be obtained with subtilisin BPN'. JOHANSEN (88) showed that deacylation of succinyltyrosyl residues in subtilisin type Carlsberg proceeded with different rates, three residues deacylating with a rate one third of that observed with the remaining six residues. Although maleylation was used by GERTLER (48) instead of succinylation because of its selectivity for lysyl residues, it cannot be excluded that certain tyrosyl residues could be in such a microenvironment that the fast hydrolysis usually observed with maleyl-tyrosyl residues does not take place. JOHANSEN (88) also has shown that the glutarylation of tyrosyl residues in subtilisin type Carlsberg inhibited the hydrolysis of clupein, gelatine and casein. Although the argument is speculative, it cannot be excluded that similar modification of one or more tyrosyl residues (possibly $\mathrm{Tyr}_{104}$ and $\left.\mathrm{Ty}_{217}\right)$ by maleic anhydride might lead to changes in the binding capacity of subtilisin BPN' $^{\prime}$ towards salmine sulfate resulting in an increase in $\mathrm{K}_{\mathrm{M}}$. 
That the introduction of negative charges in strategically important locations on the enzyme surface is the cause of enhanced proteolytic activity of the subtilisins is further substantiated by succinylation or glutarylation of subtilisin type Carlsberg (88). Although the reagents used, succinic and glutaric anhydride, are quite unspecific since they react with lysyl, tyrosyl as well as threonyl/seryl residues, it was demonstrated that the marked changes in proteolytic activity of the enzyme was caused by modification of seryl and/or threonyl residues. The changes in enzymatic behaviour were similar to but even greater than those found upon nitration or iodination. From a comparison of the distribution of negative charges and seryl/threonyl residues in the active site region of the two subtilisins it was suggested that the modification leading to the changes in enzymatic activity most likely took place at Ser $_{156}$ in the Carlsberg enzyme. In subtilisin $\mathrm{BPN}^{\prime}$ this residue is replaced by a negatively charged glutamic acid residue. If the area around residue 156 is of importance in the binding of clupein the introduction of a negative charge by succinylation or glutarylation of $\operatorname{Ser}_{156}$ in subtilisin type Carlsberg might well improve the catalytic efficiency.

The participation of $\mathrm{Glu}_{156}$ in the binding of clupein to subtilisin $\mathrm{BPN}^{\prime}$ has been suggested also from studies in which carboxyl groups were esterified (3). At a degree of modification which hardly affects the rate of hydrolysis of TAME, the rate of hydrolysis of clupein sulfate $(0.5 \%)$ is less than $15 \%$ of that obtained with the unmodified enzyme. Casein $(0.5 \%)$ is hydrolyzed with a rate $50 \%$ of that obtained with the unmodified enzyme. Thus it appears reasonable to conclude that the secondary binding of polypeptide substrates is affected. No similar studies have been performed with subtilisin type Carlsberg. If the hydrolysis of clupein sulfate in this cace turns out to be not affected by the same degrec of modification, additional support for the importance of $\mathrm{Glu}_{156}$ in subtilisin BPN' will be gained. However, it should be pointed out that $\mathrm{Asp}_{98}$ in subtilisin $\mathrm{BPN}^{\prime}$ (which is a seryl residue in the Carlsberg enzyme) appears also to be a likely candidate for participation in a subsite.
Glu $_{156}$ is not included as part of a subsite in the binding site region proposed by KRAUT and coworkers for subtilisin BPN'. However, these investigators have shown (4) that binding of the dipeptide Lys-Tyr to crystals of subtilisin BPN' takes place via a hydrogen bond between Glu $_{156}$ and the tyrosyl residue. Although this finding does not prove that productive binding takes place in this way, it at least demonstrates that the area around $\mathrm{Glu}_{156}$ is a potential binding site. If, on the other hand, the suggestion is correct that $\mathrm{Glu}_{156}$ forms part of a productive binding site, then an alternative mode of binding to that proposed by KRAUT and coworkers for polypeptides exists, and this alternate mode might be the one indicated from kinetic studies.

It is interesting to note that two of the chemical modification studies discussed above, nitration or iodination of tyrosyl residues on the one hand and succinylation or glutarylation of seryl and/or threonyl residues on the other, although qualitatively leading to the same changes in enzymatic activity, introduce functionally important negative charges at different locations on the enzyme surface. Since clupein is a large peptide of about 30 residues, it is possible that the chemical modifications alter two binding sites of the same binding mode (probably $\mathrm{S}_{4}$ and $S_{2}^{\prime}$ ). Hence, it is not necessary to postulate two different productive binding modes for clupein. However, it should be noted that nitration of glutarylated subtilisin type Carlsberg did not lead to any further changes in the rate of hydrolysis of clupein sulfate. Thus, the changes obtained by the two types of modification are not additive.

\subsubsection{Effect of Neutral Salts}

Improved binding of clupein to the subtilisins as a result of introduction of negative charges into these enzymes showed that charge-charge interaction in some way was involved. In order to get a better understanding of these interactions, experiments were performed in which increasing concentrations of various neutral salts were added to reaction mixtures of clupein sulfate and either subtilisin type Carlsberg or subtilisin $B P N^{\prime}$ (143). A tenfold decrease in $K_{m}$ (app) was calculated from Fig. 9 for both enzymes at salt 


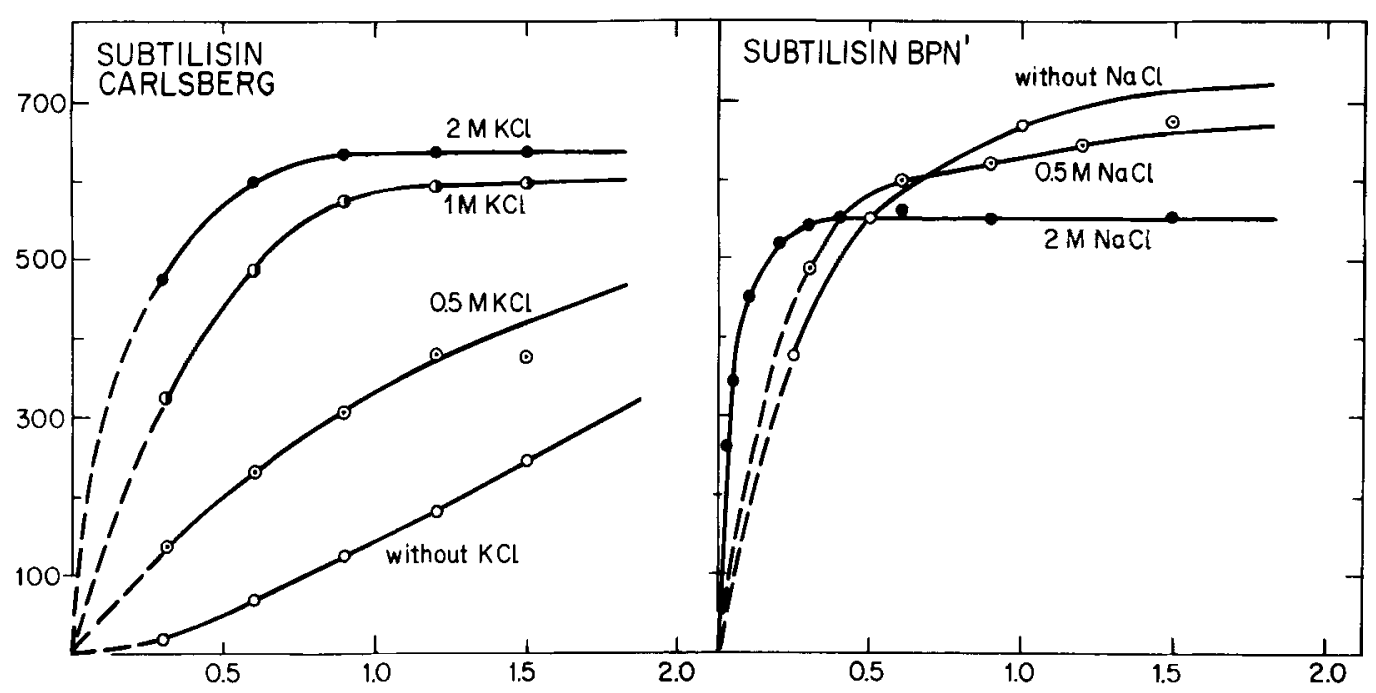

Figure 9. The effect of high concentrations of salt on the rate of hydrolysis of clupein sulfate by subtilisin type Carlsberg and $\mathrm{BPN}^{\prime}$ at $\mathrm{pH} 8.0$ and $30^{\circ} \mathrm{C}$. Ordinate: rate of hydrolysis expressed as $\mu \mathrm{eq} \mathrm{H}^{+}$liberated per min per $\mathrm{mg}$ enzyme per $\mathrm{ml}$ in a $10 \mathrm{ml}$ incubation mixture. Abscissa: per cent clupein sulfate.

concentrations of $2 \mathrm{M}$. The simplest way of explaining the salt effect is that of a general decrease in the repulsion between the enzyme and substrate both of which are positively charged. However, it is difficult to reconcile such an explanation with the observation that carbamylation (187) of almost all lysyl residues in subtilisin $\mathrm{BPN}^{\prime}$ or succinylation (88) of the lysyl residues in subtilisin type Carlsberg has no effect on the rate of hydrolysis of clupein. These chemical modifications changes the isoelectric points of the subtilisins from $\mathrm{pH}$ about 9 to about $\mathrm{pH} 4-5$. Therefore, the salt effect must be considered primarily to be a local effect.

If electrostatic attraction played a major role in the binding of substrates a decrease in hydrolytic efficiency would be expected upon salt addition. However, both the kinetic and crystallographic evidence already discussed point toward hydrophobic interactions being mainly responsible for substrate binding. Clupein having a high content of positively charged arginyl residues can be visualized as having difficulties in accomodating the peptide chain in such a way that good contact is obtained with the enzyme surface while at the same time all the positive charges in the particular segment bound point away from the enzyme surface.
In experiments with the ester substrates TAME and ATEE it was demonstrated for both enzymes that the $\mathrm{pK}$ of the prototropic group on which the activity depends increased from $\mathrm{pH}$ 7.0 to about 7.5 when the salt concentration was increased from $0.1 \mathrm{M}$ to $2.0 \mathrm{M}$. An increase in the rate of hydrolysis of TAME by subtilisin type Carlsberg was concomitantly observed while the hydrolysis by subtilisin $\mathrm{BPN}^{\prime}$ was almost unaffected. One of the effects of salt is therefore on the catalytic step. A similar conclusion was made by KAHANA and SHALITIN (91) based on the rate of hydrolysis of ATEE by subtilisin $\mathrm{BPN}^{\prime}$ in 0.1 and $2.0 \mathrm{M} \mathrm{NaCl}$, respectively. The much more pronounced effect seen with the peptide clupein can only be explained by an additional effect on the secondary binding sites for this substrate. The positioning of a positively charged arginyl residue in a hydrophobic environments is energetically very unfavorable. However, it is possible if the rest of the forces involved in the substrate binding are strong enough to counteract this. This was demonstrated in the case of pancreatic trypsin inhibitor (PTI) (76), which binds to both trypsin and chymotrypsin in the same way. Since the bottom of the $S_{1}$ "pocket" is occupied by an aspartyl residue in trypsin a strong salt bridge can be made with the functional impor- 
tant lysyl residue of PTI. In chymotrypsin, $S_{1}$ is hydrophobic throughout and will not easily accomodate a charged amino acid side-chain. However, about 400 contacts, predominantly van der Waals contacts, can be made between PTI and chymotrypsin and the association energy is therefore great enough to supply the energy needed to place the positive charge in the hydrophobic pocket. The association energy is lowered by about $4 \mathrm{kcal}$ compared with trypsin. It is possible that what we observed in the case of the subtilisins is a strengthening of the hydrophobic contacts with clupein ("salting out (1) which to a large extent overcome the repulsion of the positive charge in arginine.

That the salts primarily exert their effect on the binding of substrate is demonstrated by the observation that casein which binds well to the subtilisins without addition of salt is not hydrolysed at a different rate in the presence of salts. If salts had effected primarily the catalytic step in protein hydrolysis, similar effects would be observed with clupein and casein.

Kahana and Shalitin (91) made the interesting observation that in $2.0 \mathrm{M}-\mathrm{NaCl}$ polylysine became a good substrate for $\alpha$-chymotrypsin. However, this substrate was not degraded lower than to octamers. These authors suggested that the high salt concentration led to formation of new binding modes for the substrate. A similar observation was made by BRETZEL (25) in the case of pepsin, clupein is not digested by this enzyme in the absence of salt, but becomes a substrate in $2 \mathrm{M}-\mathrm{NaCl}$.

\subsubsection{Effects of Alcohols and Guanidine Hydro- chloride}

Further evidence for the dominance of hydrophobic interactions for enzyme-substrate complex formation by the subtilisins was provided by experiments in which alcohols, various organic solvents and guanidine hydrochloride were added to the digestion mixture (189).

In one series of experiments straight chain, aliphatic alcohols ranging from methanol to pentanol were added in increasing amounts to reaction mixtures of subtilisin type Carlsberg or subtilisin BPN' and ATEE, TAME, or clupein sulfate. In all cases inhibition was observed, but

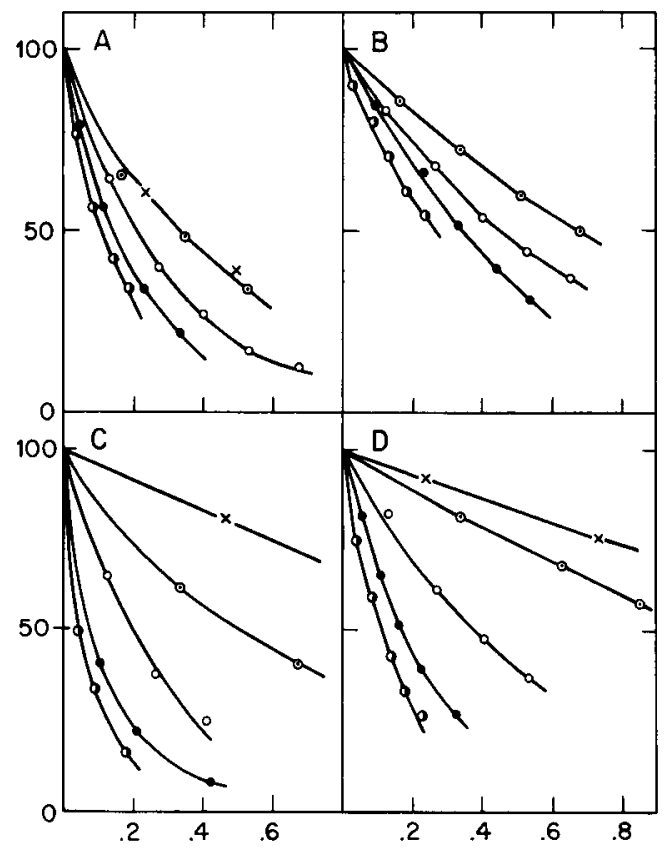

Figure 10. Hydrolysis af ATEE and clupein sulfate by the subtilisins in the presence of straight-chain, aliphatic alcohols. A: subtilisin type Carlsberg and 2.5 mM ATEE, B: subtilisin BPN' and $2.5 \mathrm{mM}$ ATEE, C: subtilisin type Carlsberg and $0.45 \%$ clupein sulfate, D: subtilisin $\mathrm{BPN}^{\prime}$ and $0.45 \%$ clupein sulfate. $\mathrm{pH}=$ $8.0, \mathrm{t}=30^{\circ} \mathrm{C}$. Abscisse: alcohol concentration (moles/1). Ordinate: relative rate of hydrolysis.

$-x$ - methanol, $-\odot-$ ethanol, $-0-$ propanol, - - butanol, - - pentanol.

it was considerably stronger when clupein sulfate was the substrate (Fig. 10). If $S_{1}$ was the only important binding site for both the ester substrates and clupein sulfate one would expect that the more strongly bound substrate would be influenced less by addition of alcohols. However, clupein sulfate is bound about ten times better than TAME to subtilisin type Carlsberg $\left(\mathrm{K}_{\mathrm{M}}\right.$ is $5 \times 10^{-3} \mathrm{M}$ and $4 \times 10^{-2} \mathrm{M}$, respectively (143)). With subtilisin $\mathrm{BPN}^{\prime}$ the difference is even more pronounced $(\sim$ $8 \times 10^{-4} \mathrm{M}$ for clupein sulfate and $2.5 \times 10^{-2} \mathrm{M}$ for TAME). The alternative explanation, that hydrolysis of clupein sulfate is inhibited more strongly than that of the ester substrates because clupein interacts with additional subsites on the enzyme surface appears more likely. Since the subsites identified by KRAUT and coworkers are hydrophobic (the wsticky 
spots" $S_{1}^{\prime}$ and $S_{2}^{\prime}$, the hydrophobic pocket in $S_{4}$ and the hydrophobic crevice in $S_{1}$ ), binding of alcohols to these subsites seems likely. A pronounced increase in inhibitory power was observed in going from methanol to pentanol (Fig. 10). This observation is compatible with the difference in both size and hydrophobicity between the two alcohols.

The observation that the logarithm of relative rate of hydrolysis always gave a straight line when plotted against alcohol concentration (189) was explained in terms of multiple binding sites for the inhibitors in a theoretical treatment of RALSTON (151).

No inhibition by alcohols was observed when casein, even at concentrations as low as $0.09 \%$, was hydrolyzed by subtilisin type Carlsberg. The $\mathrm{BPN}^{\prime}$ enzyme was inhibited only weakly by $\mathrm{n}$-pentanol and n-hexanol. These results are compatible with a much better multiple site binding of casein $\left(\mathrm{K}_{\mathrm{M}}(\mathrm{app}) \sim 10^{-5} \mathrm{M}\right)$ compared to that of clupein sulfate.

Competitive inhibition by dimethylformamide has been observed by MORIHARA et al. (124) in the hydrolysis of Z-Gly-Leu- $\mathrm{NH}_{2}$ by subtilisin $\mathrm{BPN}^{\prime}$. Thus $\mathrm{K}_{\mathrm{M}}$ increased 10 fold in $15 \%$ dimethylformamide over that in uninhibited solutions. In the comparative study of the hydrolysis of a series of peptides by subtilisin $\mathrm{BPN}^{\prime}$ the reaction mixtures always contained $15 \%$ dimethylformamide in order to keep the substrates in solution. Such a procedure presupposes that the binding of all peptide substrates is affected to the same degree by $15 \%$ dimethylformamide. This is not necessarily the case as demonstrated by the hydrolysis of clupein and casein, and care should be taken in interpretation of experiments in which organic solvents of various kinds have been added with the purpose of keeping the substrate in solution. It has been shown (189) that, in addition to the alcohols discussed above, subtilisin type Carlsberg is inhibited to a varying degree by acetonitril $<$ dioxane $<$ tert-butanol $<$ n-butyric acid $<$ valeric acid, cyclohexane.

Guanidine hydrochloride which acts as a denaturing agent was also added to reaction mixtures of the subtilisins and various substrates (189), but in concentrations at which the enzymes are still in their native state. Inhibition patterns similar to but not identical with those obtained with aliphatic alcohols were observed. Thus the inhibition of ATEE and TAME hydrolysis was more pronounced with subtilisin BPN'. The action of subtilisin BPN' on clupein sulfate was also inhibited more than that of the Carlsberg enzyme, but the enzymatic activity of the latter was increased at low concentrations of guanidine hydrochloride probably due to the function of this compound both as an "activating" salt and as an inhibitor (143). The hydrolysis of casein by both enzymes was totally unaffected by addition of guanidine hydrochloride even in concentration as high as $2.5 \mathrm{M}$, again demonstrating that the inhibitor affects the binding step rather than the hydrolytic step.

\section{BINDING SITES IN OTHER PROTEASES}

The increasing number of publications dealing with the size of the active site of proteases clearly demonstrates that the importance of secondary binding sites is being fully recognized. In most studies synthetic polypeptides have been used and, in those cases where the three-dimensional structure of the enzyme is known, studies similar to those described for subtilisin BPN' have been performed. Since the hydrolytic mechanism appears to be identical in the subtilisins and the phylogenetic unrelated mammalian serine proteases it is of interest to investigate how far the similarities extend also with respect to secondary interactions with substrates. In this section, therefore, the secondary binding sites of chymotrypsin, trypsin, and elastase are discussed, but many examples of secondary interactions in other proteases are included as well in order to give a more comprehensive picture of the similarities and differences in the interaction between proteases and peptide substrates.

\subsection{Chymotrypsin}

The high specificity of chymotrypsin for substrates having aromatic amino acids in $P_{1}$ is well documented (67), but several investigations have also been performed with respect to the secondary binding properties of this enzyme. 
YosHiDA et al. (220) studied the hydrolysis of ester substrates of the general formula $\mathrm{Gly}_{(\mathrm{n})^{-}}$ Tyr ${ }^{\ddagger} \mathrm{OEt}$ and peptides of the general formula Gly $_{(n)}-\operatorname{Tyr}^{\ddagger} \mathrm{Gly}_{(\mathrm{n})}(\mathrm{n}=1-4)$. It was shown that increase in chain length led to changes in rate of hydrolysis. Maximal rate was obtained with the peptide $\mathrm{Gly}_{(2)}-\mathrm{Tyr}_{\mathrm{t}} \mathrm{Gly}\left(\mathrm{y}_{(4)}\right.$ among the peptides tested, but the ester substrates were always hydrolyzed with a much faster rate. Since the terminal groups were not blocked in the peptides the increase in rate of hydrolysis was explained mainly by the decrease in electrostatic interaction with the catalytic site as the chain became longer. However, it should be noted that the ester substrate $\mathrm{Gly}_{(2)}-\mathrm{Tyr}^{\mathrm{f}}-\mathrm{OEt}$ was hydrolyzed much faster than both $\mathrm{Gly}_{(3)}$ Tyr $=\mathrm{OEt}$ and $\mathrm{Gly}_{(4)}$-Tyr $-\mathrm{OEt}$, the decrease in rate being reflected almost entirely in $K_{M}$.

MORIHARA et al. (123) have investigated both side-chain and stereospecificity of a-chymotrypsin. Stereospecificity was observed in five subsites $\left(S_{1}-S_{3}\right.$ and $\left.S_{1}^{\prime}, S_{2}^{\prime}\right)$ indicating that the active site covered at least $18 \dot{A}$. The side-chain specificity was compared with that of subtilisin BPN' and interesting differences were noted. While Ala in $P_{2}$ gave optimal conditions for hydrolysis by subtilisin $\mathrm{BPN}^{\prime}$ a decrease in rate was observed with chymotrypsin when Ala was substituted for Gly in Ac-Ala-Tyr $\stackrel{N_{2}}{N_{2}}$. Another difference was noted in $S_{1}^{\prime}$. The hydrolysis of Z-Tyr-Tyr $=\mathrm{NH}_{2}$ was the fastest among the substrates tested with chymotrypsin, while it was resistant to attack by subtilisin BPN'.

Crystallographic studies on chymotrypsin A $\gamma$ which had reacted with peptide chloromethyl ketones have been performed by SEGAL et al. (173). Similar to what was found with subtilisin, the contacts between enzyme and substrate formed an antiparallel $\beta$-pleated sheet involving $\operatorname{Ser}_{214}, \operatorname{Trp}_{215}$, and $\mathrm{Gly}_{216}$ in chymotrypsin. Three hydrogen bonds, two of which were connected to $P_{3}$ and one to $P_{1}$, were formed. Since both the $\mathrm{C}=\mathrm{O}$ and $\mathrm{NH}$ of $\mathrm{P}_{2}$ pointed away from the enzyme, and the corresponding groups on the enzyme pointed towards the interior, no hydrogen bonds were formed with $\mathrm{P}_{2}$ :

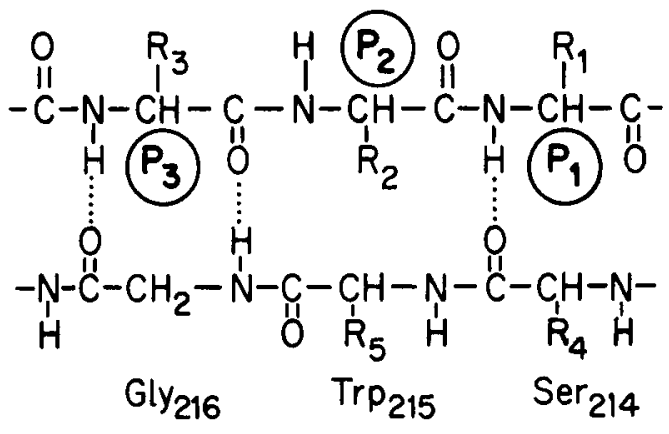

These observations are in remarkable agreement with what was found for subtilisin BPN $^{\prime}$ by KRAUT and coworkers (98). They rotated the coordinates of 27 atoms for the catalytic residues Ser, His, and Asp and the back-bone atoms of $S_{1}, S_{2}$, and $S_{3}$ in chymotrypsin until the best least square fit to the same atoms in subtilisins BPN' $^{\prime}$ was obtained. The average difference in atom position was $1.0 \AA$ with a maximal difference of $2.1 \dot{\AA}$. The close resemblance is indeed impressive, but since the subsites in the two enzymes include many other side-chains the differences in specificity of $S_{2}$ and $\mathrm{S}_{4}$ (see below) is understandable since no homology exist between the mammalian and bacterial enzymes. Furthermore, nothing has been deduced from the crystallographic studies about the subsites $S_{1}^{\prime}$ and $S_{2}^{\prime}$ in chymotrypsin observed by NEIL et al. (130) and MORIHARA et al. (123). It would be interesting to know if the similarities found with $S_{1}, S_{2}$, and $S_{3}$ also extend to $S_{1}^{\prime}$ and $S_{2}^{\prime}$.

Crystallographic studies also show that the antiparallel $\beta$-pleated sheet is not expected to extend beyond $\mathrm{Gly}_{216}$ since a sharp bend of the enzyme back-bone away from the inhibitor is observed at $\mathrm{Gly}_{216}$. However, kinetic studies of the inhibition of chymotrypsin with peptide chloromethyl ketones (99) have shown that if a $\mathrm{Z}$ or Boc moiety were placed in $\mathrm{P}_{4}$ the rate if inactivation was decreased. This was explained by an interaction of the hydrophobic groups in $\mathrm{P}_{4}$ with $\operatorname{Trp}_{172}$ on the enzyme. This interaction was probably so strong that the hydrogen bond between $S_{3}$ and $P_{3}$ was disrupted which in turn led to decrease in the rate of reaction with the essential histidine. A big difference is observed between chymotrypsin and subtilisin with respect to $S_{4}$. As discussed in section 3.4 this 
site is important in the latter enzyme. In contrast to what was found with chymotrypsin good contacts at this site means more efficient catalysis.

In addition to preference of $S_{1}$ in chymotrypsin for a Tyr, the crystallographic study (173) also explained the specificity of $\mathrm{S}_{2}$ for aliphatic residues having a large, bulky side-chain. This had already been demonstrated by NEIL et al. (130) who showed that Val, Ile, Lys, and Pro in $\mathrm{P}_{2}$ favoured hydrolysis. The $\mathrm{X}$-ray data showed that good van der Waal's contacts could be obtained between a Leu in $\mathrm{P}_{2}$ and the enzyme. The deductions made from crystallographic studies were reinforced by kinetic studies with either peptide chloromethyl ketones (99) or Nacetyl peptide methyl esters (174). Thus a Leu in $P_{2}$ increased the rate of inactivation with chloromethyl ketones substantially (99). However, a tosyl group in this position made the inhibitor slightly less effective than one having an acetyl group. In subtilisin maximal enzymatic efficiency was obtained with an Ala in $\mathrm{P}_{2}$.

In the studies on chymotrypsin discussed above only one binding mode for peptides has been observed crystallographically as well as kinetically. This may be the result of the very strong specificity of subsite $S_{1}$ : having $P_{1}$ always fixed in the same position restricts the possibilities of the following residues for binding to alternative binding sites.

\subsection{Trypsin}

The first report on hydrolysis of synthetic peptides by trypsin was published in 1959 by IZUMIYA and UCHIO (81). Peptides of the general formula $\mathrm{Gly}_{(\mathrm{n})^{-}}$Lys $-\mathrm{NH}_{2}(\mathrm{n}=1-4)$ were used as substrates. Large increases in $\mathrm{k}_{\text {cat }} / \mathrm{K}_{\mathrm{M}}$ were observed with Gly-Gly-Lys $\mathrm{NH}_{2}$ relative to Gly-Lys $\mathrm{NH}_{2}$. However, Gly-Gly-Gly-Lys${ }^{-} \mathrm{NH}_{2}$ was hydrolyzed with the same rate as GlyGly-Lys $\_\mathrm{NH}_{2}$. Extension of the peptide-chain by one more Gly to Gly-Gly-Gly-Gly-Lys $\leqslant \mathrm{NH}_{2}$ resulted in a decrease in hydrolysis by a factor of two.

In a subsequent work YAMAMOTO and IZUMIYA (219) investigated the influence of chain elongation at the C-terminal end by means of substrates of the type Gly-Gly-Lys $\_G l y(n)(n=1-4)$. While Gly-Gly-Lys $\_$Gly was a very poor sub- strate, probably due to the $\mathrm{COO}^{-}$group of Gly, addition of one or two glycyl residues raised $\mathrm{k}_{\mathrm{ca}} / \mathrm{K}_{\mathrm{M}}$ by a factor of 140 and 700 , respectively. Addition of yet another Gly decreased $\mathbf{k}_{\text {cat }} / \mathrm{K}_{\mathrm{M}}$ relative to $(\mathrm{Gly})_{2}-\mathrm{Lys} \_(\mathrm{Gly})_{3}$. Thus, trypsin was found to have at least seven subsites for binding peptides.

Although the three-dimensional structure is known (196), very few experimental data have been published on the binding of large substrates to crystals of trypsin. However, it is reasonable to expect results similar to what was found with chymotrypsin. First, trypsin is very specific with regard to Lys or Arg which probably restricts the number of binding modes to one only. Second, the segment Gly ${ }_{216}-\operatorname{Trp}_{215}-$ $\mathrm{Ser}_{214}$ shown to form antiparallel $\beta$-sheet type contacts with peptide substrates in chymotryp$\sin$ is identical in trypsin. In addition, the possibility of formation of a fourth hydrogen bond exists due to a deletion at residue 218. By model building, STroud et al. (196) showed that the binding of pancreatic trypsin inhibitor (PTI) involved the segment $\mathrm{Gly}_{216}-\mathrm{Tyr}_{215}-\mathrm{Ser}_{214}$. A similar result was obtained by HUBER et al. (75) with chymotrypsin, which is also inhibited strongly by PTI. As pointed out by HUBER, the contribution to the binding energy from the hydrogen bonds may be small, the main contribution coming from van der Waal's contacts, but the hydrogen bonds may be important for the proper alignment of the inhibitor. By analogy, one could expect the subtilisins also to bind PTI. Indeed, a model building study of RoBertus et al. (160) demonstrated a remarkably good fit of the PTI recognition site into the binding channel of subtilisin BPN'. However, PTI is not bound by subtilisin (76). According to KRAUT and coworkers (160) this is due to overlap of the inhibitor with a loop on the enzyme outside the binding channel.

\subsection{Elastase}

The secondary binding sites of elastase were investigated by BERGER and coworkers (8). They showed that six to seven subsites $\left(S_{1}-S_{4}\right.$ and $S_{1}^{\prime}$, $\left.S_{2}^{\prime} / S_{3}^{\prime}\right)$ were operative. Subsite $S_{4}$ was found to be very important. In going from Ala-Ala-Ala Lys-Phe to Ala-Ala-Ala-Ala-Lys-Phe, an increase in $k_{\text {cat }} / K_{M}$ of 1440 was observed with a 
Hydrolysis of some synthetic peptides by elastase.

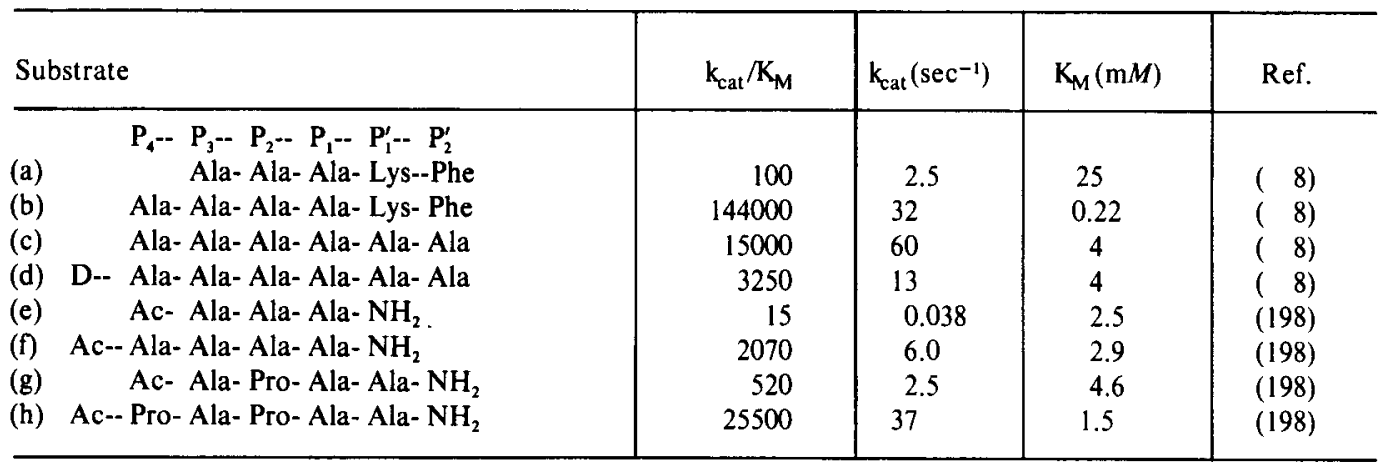

large contribution from a decrease in $\mathrm{K}_{\mathrm{M}}$ and a smaller contribution from an increase in $k_{\text {cat }}$ (a and $b$ in Table VIII). However, the stereospecificity of $\mathrm{S}_{4}$ was reflected exclusively in $\mathrm{k}_{\text {cat }}$ (c and $d$ in Table VIII). The importance of Lys and $P$ he in subsites $S_{1}^{\prime}$ and $S_{2}^{\prime}$ was demonstrated by step-wise exchange of these residues by Ala leading to a 10 fold decrease in $\mathrm{k}_{\mathrm{cat}} / \mathrm{K}_{\mathrm{M}}$. It was further concluded that the importance of Lys in $\mathrm{P}_{1}^{\prime}$ was due to the hydrophobic part of the sidechain and not to the $\varepsilon$-amino group. Trifluoroacetylation of this group had no qualitative effect on the hydrolysis of the AlaLys bond.

THOMPSON and BLOUT (197) also concluded that binding of the substrate in $\mathrm{S}_{4}$ was of great importance for hydrolysis of ester, amide, and peptide substrates. However, an analysis of the kinetic data showed that the primary effect of chain elongation was to increase the rate of acylation (Table VIII, e-h). The kinetic data were best explained by an ninduced fit" (97). This means that binding at $\mathrm{P}_{4}$ alters the enzyme conformation to give a more favourable alignment of $P_{1}-P_{1}^{\prime}$ at $S_{1}-S_{1}^{\prime}$. In principle, this could also be obtained by a movement of the whole substrate chain, but such an explanation was discarded (199).

Further evidence for the importance of $S_{4}$ was given by ATLAS and BERGER (9) who studied the hydrolysis of p-nitrobenzyl esters of various peptides. However, it was shown that Ala-AlaAla-Leu-ONbz was a better substrate than AlaAla-Ala-Ala-ONbz, which at first sight appeared puzzling in view of the preference of
$S_{1}$ for Ala. A separation of $k_{\text {cat }} / K_{M}$ into the two constants showed that the Leu derivative was bound less tightly than the Ala derivative, but that $\mathrm{k}_{\mathrm{cat}}$ of the former was much higher. With Ala or Val in $P_{1}, k_{\text {cat }}$ did not change with an increase in chain length, but a sharp increase was observed with Leu, Gly, or Phe in $P_{1}$. These observations were tentatively explained by strain on the scissil bond if the side-chain of $P_{1}$ did not fit well into $S_{1}$. The necessary activation energy was thereby lowered and $\mathbf{k}_{\text {cat }}$ increased. However, a tightly anchored residue in $S_{1}^{\prime}$ (the Nbz moiety) was also necessary. If this group was replaced by the dipeptide Lys-Ala the hydrolysis proceded "normally", that is, AlaAla-Ala-Ala Lys-Ala was hydrolyzed much faster than Ala-Ala-Ala-Leu-Lys-Ala. These results show that wrong conclusions can be made about specificity if too few substrates are investigated.

Kinetic studies on the inhibition of elastase by peptide chloromethyl ketones (201) also demonstrated the dependence of hydrolytic rate on chain-length.

From studies on the hydrolysis of tri- and tetrapeptide amides THOMPSON and BLOUT (202) concluded that the effect of $\mathrm{P}_{4}$ on both binding and hydrolytic rate could be separated. The effect on binding arose mainly from the interaction between enzyme and side-chain of the amino acid in $\mathrm{P}_{4}$, while the effect on rate mainly resulted from interaction between enzyme and the amino acid $\alpha$-amino group.

The binding of peptide inhibitors to crystals of elastase has been studied by SHOTTON et al. 
(179). Unexpectedly, it was found that these inhibitors were bound in a way different from that observed with chymotrypsin and subtilisin. While Ala-Ala-Ala and Ac-Pro-Ala-Pro-Ala were bound in one way only (occupying subsites $\mathrm{S}_{1}-\mathrm{S}_{3} / \mathrm{S}_{4}$ ), Ac-Ala-Ala-Ala was bound in two ways, one similar to that observed with the two other inhibitors, the other occupying $\mathrm{S}_{2}-\mathrm{S}_{4}$ and resembling the mode of binding of peptide substrates to chymotrypsin. The importance of $\mathrm{S}_{4}$ was tentatively explained from observed movements of side-chains which were part of $S_{3}$ and $S_{1}$ upon binding of inhibitors. Binding of the peptide Lys-Phe in subsites $S_{1}^{\prime}$ and $S_{2}^{\prime}$ was not observed although this was expected from the finding that Ala-Ala-Ala-Ala-Lys-Phe is a good substrate and Lys-Phe is a good competitive inhibitor.

A similarity between subtilisin and elastase is found in the importance of subsite $S_{4}$, a site which is of minor importance in chymotrypsin and trypsin. While a major contribution to the binding energy in chymotrypsin and trypsin probably arises from the perfect fit of the sidechain of $P_{1}$ in the "pocket " $\left(S_{1}\right)$, this will hardly be the case for subtilisin and elastase. In subtilisin the $S_{1}$ is much more open than in chymotrypsin and trypsin, and in elastase the entrance to the "pocket " is blocked by a Val (210). The best fit to $S_{1}$ is obtained by Ala which therefore to a large extent determines the specificity of elastase. However, the relative weak forces of binding give other subsites a possibility of playing an important role as well.

\subsection{Carboxypeptidase}

The earliest reported investigation of the influence of substrate chain length on the rate of hydrolysis was made by IZUMIYA and UCHIO (82) who studied the hydrolysis of the homologous series $\mathrm{Gly}_{(\mathrm{n})}{ }^{\downarrow} \mathrm{Tyr} \quad(\mathrm{n}=1-5)$ by carboxypeptidase. While Gly-Tyr was hydrolyzed very slowly, addition of one more Gly to the $\mathrm{N}$-terminal end led to an increase in $\mathrm{k}_{\mathrm{cat}} / \mathrm{K}_{\mathrm{M}}$ of 200 . Further addition of Gly to the chain had no effect.

However, BERGER and coworkers $(1,171)$ showed that the active site could be divided into at least 5 subsites $\left(S_{4}\right.$ to $\left.S_{1}^{\prime}\right)$ extending over about $18 \AA$. It was concluded that the active site as a whole was hydrophobic since a benzyl group, independent of which position it occupied, always led to a decrease in $\mathrm{K}_{\mathrm{M}}$. On the other hand exchange of L-amino acids with D-amino acids only had a minor effect on $\mathrm{K}_{\mathrm{M}}$, while $k_{\text {cat }}$ decreased between 3 and 50 fold.

Like the endopeptidases, carboxypeptidase A functions both as a peptidase and as an esterase. No difference has been observed in the binding modes of peptides and esters to the endopeptidases, but such a difference exists in carboxypeptidase $\mathrm{A}$, and a model for substrate binding taking this into account has been proposed (207). The conclusions about the difference in substrate binding were based on studies with modifiers which influenced the rate of hydrolysis of peptides and esters differently (36) and on chemical modifications of the enzyme. Thus, acetylation of tyrosyl residues with either $\mathrm{N}$ acetyl imidazole or acetic anhydride $(154,180)$ increased esterase activity and greatly decreased peptidase activity. Similar results were obtained by modification of a single tyrosyl residue with tetranitromethane (157). In agreement with the proposed model for substrate binding it was found that the hydrolysis of peptides like Cbz-Gly-Gly ${ }^{\downarrow}$ Phe and Bz-GlyGly $₫$ Phe was inhibited non-competitively by competitive inhibitors of ester hydrolysis.

A new technique for kinetic studies as well as for distance measurements at the active site has been introduced by LATT et al. (101). It is based on the use of spectrochemical probes and has the advantage that distances can be measured while the enzymatic reaction is in progress thus making sure that it is productive binding which is observed. It was found that the distance between the Co atom in the catalytic center of Co-carboxypeptidase and the probe (a Nterminal dansyl group) was less than $8 \AA$ for dipeptides and about $18 \AA$ for pentapeptides. Substrate fitting to the three-dimensional model agreed with the measured distances when the peptides were in their extended form. Maximal catalytic efficiency was obtained with the tripeptides, while a decrease in $\mathrm{k}_{\text {cat }}$, but not in $K_{M}$ was observed with the tetra- and pentapeptides used in this study.

In a recent study ABRAMOWITZ-KURN and SCHECHTER (2) have demonstrated good agree- 
ment between results obtained from the hydrolysis of numerous synthetic substrates and a proposed model for substrate binding based on model building.

\subsection{Pepsin}

In a series of papers FRUTON and coworkers $(46,117,166,215)$ have elucidated the binding of oligopeptide substrates and inhibitors to pepsin. These authors do not use the term "subsites", which in their opinion involves the assumption that wthese loci of interaction are associated with a relative rigid structure of the binding area of pepsin“ (73). Also the terms "primary" and "secondary binding sites" are used to designate binding of the substrate molecule as a whole at the active site and at other regions on the enzyme surface, respectively (78). Oligopeptides are described by the general formula $\mathrm{A}-\mathrm{X}-\mathrm{Y}-\mathrm{B}$, where $\mathrm{X}$ and $\mathrm{Y}$ are the residues adjacent to the susceptible bond and $\mathrm{A}$ and $\mathrm{B}$ the rest of the amino acids and terminal blocking groups on the $\mathrm{N}$ - and $\mathrm{C}$-terminal side, respectively. A and B are treated as units.

The determination of the catalytic parameters $K_{M}$ and $k_{c a t}$ for a series of substrates showed that the difference in reactivity rested almost exclusively in $k_{\text {cat }}$ while only small changes in $\mathrm{K}_{\mathrm{M}}$ were noted. The explanation appears to be that the major contribution to the binding comes from $\mathrm{X}$ and $\mathrm{Y}$ as long as one of these residues is aromatic. Table IX shows data that illustrate the importance of secondary binding of the substrate. Thus, prolongation of the peptide chain by one and two glycyl residues towards the $\mathrm{N}$-terminal end ( $\mathrm{b}$ and $\mathrm{c}$ ) increased $\mathrm{k}_{\text {cat }}$ relative to (a) by a factor of 4,5 and 115 , respectively. The exchange of the Gly's by Ala's had also a remarkable effect on $k_{c a t} / K_{M}$ (d). An interesting specificity for cationic sidechains is illustrated in (e) to $(\mathrm{g})$. The hydrolysis of (e) and (f) was pH-dependent while that of (g) was not, and the change in position of Gly and His had a pronounced effect on $k_{\text {cat }}$. (h) to (k) illustrate the importance of the secondary interactions on the C-terminal side of the susceptible bond. While a moderate increase in catalytic efficiency is observed by blocking the Ala in $\mathrm{P}_{2}^{\prime}$ by methylation, a large increase occurs on addition of one more methylated Ala. Non-productive binding was observed in several of the studies. Direct binding studies were performed with the inhibitor Z-His$\mathrm{Phe}\left(\mathrm{NO}_{2}\right)-\mathrm{Pol}(\mathrm{Pol}=\mathrm{Phe}$ in which the $-\mathrm{COOH}$ group is replaced by a $-\mathrm{CH}_{2} \mathrm{OH}$ group). More than 5 molecules were bound per mole of enzyme (78), but with different strength. The most strongly bound molecule was assumed to occupy the region around the catalytic group(s) on the enzyme, the others bound at sites outside the catalytic region. Some of these binding sites were abolished upon acetylation of the enzyme with acetylimidazole. Since acetylation also led

Table IX

Hydrolysis of some synthetic peptides by pepsin.

\begin{tabular}{|c|c|c|c|c|c|c|}
\hline & & & $\mathrm{k}_{\text {cat }}$ & $\mathrm{K}_{\mathrm{M}}$ & $\mathrm{k}_{\text {(cat) }} / \mathrm{K}_{\mathrm{M}}$ & \\
\hline Substrates & & & $\left(\sec ^{-1}\right)$ & $(\mathrm{mM})$ & & Ref. \\
\hline$P_{4^{--}} \quad P_{3^{--}}$ & $-\quad P_{2--} \quad P_{1}-\cdots-\cdots--P_{1}^{\prime--} \quad P_{2--}^{\prime}$ & $\mathrm{P}_{3}^{\prime--} \quad \mathrm{P}_{4}^{\prime}$ & & & & \\
\hline (a) & Z-- Phe--..---Phe- OP4P & 1) & 0.49 & 0.71 & 0.69 & $(165)$ \\
\hline Z--- & Gly- Phe---.-.Phe- OP4P & & 2.2 & 1.1 & 2.0 & (165) \\
\hline (c) Z-- Gly-- & Gly- Phe---..-Phe- OP4P & & 56.5 & 0.8 & 70.6 & (165) \\
\hline (d) Z-- Ala-- & - Ala- Phe------Phe- OP4P & & 260. & 0.04 & 6500. & (165) \\
\hline (e) & His- Phe------Phe- OEt & & 0.47 & 0.18 & 2.65 & ( 73) \\
\hline (f) Z-- Gly-- & His- Phe------Phe- OEt & & 2.62 & 0.34 & 7.76 & ( 73) \\
\hline (g) Z-- His-- & - Gly-Phe--_-Phe- OEt & & 0.03 & 0.17 & 0.17 & ( 73) \\
\hline Z--- & His-Phe $\left(\mathrm{NO}_{2}\right)$-Phe-Ala & & 2.9 & 0.8 & 3.6 & (117) \\
\hline Z-- & His--Phe $\left(\mathrm{NO}_{2}\right)$-Phe-Ala- & $\mathrm{OMe}$ & 3.3 & 0.4 & 8.3 & (117) \\
\hline (k) & His- $\mathrm{Phe}\left(\mathrm{NO}_{2}\right)$-Phe-Ala- & Ala- $\mathrm{OMe}$ & 28. & 0.13 & 215 & (117) \\
\hline
\end{tabular}

1) $\mathrm{OP} 4 \mathrm{P}=3-\left(4-\right.$ pyridyl)propyl-1-oxy, $\mathrm{Phe}\left(\mathrm{NO}_{2}\right)=$ p-nitro-L-phenylalanine 
to a diminished rate of hydrolysis of proteins (165), it was concluded that some of these secondary sites for the inhibitor were also binding sites for parts of a big molecule like hemoglobin. The catalytic site, however, is not abolished since the hydrolysis of small substrates like Z-His-Phe( $\left.\mathrm{NO}_{2}\right)$-Phe-OMe were actually increased in rate, probably due to favourable changes of the structure in the catalytic site.

Hydrolysis of synthetic peptides has also been studied by OKA and MORIHARA (134). They demonstrated that the enzyme was able to accomodate at least seven amino acid residues. In these studies $S_{1}$ was always occupied by $P$ he. The enzyme responded to changes in $P_{2}$ to $P_{4}$ and $P_{1}^{\prime}$ to $P_{3}^{\prime}$. In contrast to FRUTON and coworkers (73) these authors found that the enzyme was stringently stereospecific for the amino acid residues in $P_{2}$ and $P_{3}$. Stereospecificity was observed for $P_{2}^{\prime}$, but not for $P_{3}^{\prime}$.

\subsection{Chymosin (Rennin)}

When $\kappa$-casein is hydrolyzed by chymosin the only attack is on a Phe-Met bond whereby para- $\kappa$-casein and $\kappa$-casein macromolecules are formed (152). This finding suggested a high specificity for Phe-Met bonds, but the peptide methyl esters Phe-Met-OMe, Phe-Met-Ala$\mathrm{OMe}$, and Leu-Phe-Met-Ala-OMe were hydrolyzed only to a negligible extent (70). These results showed that the mere existence of the dipeptide, even with the end-groups blocked, was not enough for hydrolysis to take place. The same conclusion was reached by VOYNICK and FRUTON (215).

RAYMOND et al. (152) synthesized a series of peptide methyl esters closely resembling the structure around the susceptible Phe-Met bond in $\kappa$-casein: -Leu-Ser-Phe-Met-Ala- and studied the hydrolysis of these by chymosin. The results can be summarized as follows: Only bonds of the type Phe-X or Phe $\left(\mathrm{p}-\mathrm{NO}_{2}\right)-\mathrm{X}$ were hydrolyzed. Replacement of Met by Ile was without importance. On the other hand, replacement of Ser by Ala led to a drastic decrease in the rate of hydrolysis. The effect of this subtle change (replacement of a $-\mathrm{OH}$ group by a $\mathrm{H}$ ) can hardly be related to the change in size of the side-chain, especially since the rate of hydrolysis decreased. Most likely an important hydrogen bond could no longer be formed between substrate and enzyme. The same effect was found with a peptide in which the $\mathrm{CH}_{2} \mathrm{OH}$ side chain of Ser was replaced by a $\mathrm{NH}_{2}$ group. In this case hydrogen bonding should in principle be possible, but the distance from the amino nitrogen may be too long, or if a hydrogen bond in fact was formed, this led to distortion in the catalytic site large enough to impair hydrolysis. Finally, a very dramatic increase in catalytic efficiency was observed when the peptide chain was extended by one more residue, a Leu at the C-terminal end. In the terminology of SCHECHTER and BERGER the substrate recognized at least 5 subsites $\left(S_{2}\right.$ to $S_{3}^{\prime}$ ) on the enzyme. In those cases where the kinetic parameters $k_{\text {cat }}$ and $K_{M}$ were determined, the major effect of the changes in amino acid side-chains was observed on $\mathrm{k}_{\mathrm{cat}}$ while only minor changes were found with $\mathrm{K}_{\mathrm{M}}$. It was concluded that the rapid preferential cleavage of the Phe-Met bond in $\mathrm{K}$ casein was a consequence of secondary interactions between substrate and subsites on the enzyme.

Preliminary results have been published by SCHATTENKERK et al. (169) from studies similar to those discussed above. These authors extended the substrate so that a larger portion of the $x$-casein chain was covered:

-Leu-Ser-Phe-Met-Ala-Ile-Pro-Pro-Lys-

The results were only qualitative but in agreement with those discussed above. It was found that reversal of the sequence Leu-Ser in the pentapeptide Leu-Ser-Phe-Met-Ala- led to a drastic decrease in rate of hydrolysis.

\subsection{Thrombin}

Thrombin shows a remarkable specificity in its action on fibrinogen. The only bonds cleaved are four Arg-Gly bonds located in the $\alpha(A)$ and $\beta(B)$ chains of the hexamer $[\alpha(A) \quad \beta(B) \gamma]_{2}$ $(14,17)$. The hydrolysis of the two $\alpha(A)$ Arg-Gly bonds leads to blood clotting while the rate of hydrolysis of the two $\beta(B)$ Arg-Gly bonds first becomes maximal when the formation of fibrin is complete (18). The Arg-Gly bonds of insulin, B-chain of insulin (10), glucagon (51) or hen eggwhite lysozyme (50) are not cleaved. The hydrolysis of the Arg-Gly bonds in isolated a(A) 
and $\beta(B)$ chains show that the three-dimensional structure of fibrinogen is not needed for the specific action of thrombin (68). Therefore, it was assumed that the specificity must be determined by the sequence on both sides of the susceptible bond. In order to test this possibility SCHERAGA and coworkers (6) synthesized peptides of varying length having the correct sequence around the scissile bond, the longest peptide being

Gly-Gly-Gly-Val-Arg-Gly-Pro-Ala- $\mathrm{NH}_{2}$

It was observed that thrombin acted both as an endopeptidase and as an aminopeptidase (Arg Gly-Pro-Ala- $\left.\mathrm{NH}_{2}\right)$, and that $\left(\mathrm{NO}_{2}\right)$ Arg-Gly bonds were also hydrolyzed showing that the positive charge of the Arg side-chain was not essential for hydrolysis. It was suggested that the specificity was connected with the sequence Gly-Gly-Gly in the $\alpha(A)$ chain. These three residues in series might permit the entry of the chain into a narrow active site cleft in thrombin. More bulky side-chains in $\mathrm{P}_{3}-\mathrm{P}_{5}$ might not be accomodated and hydrolysis therefore not take place. However, in a subsequent paper (102) it was demonstrated in a more detailed kinetic analysis that $S_{1}-S_{3}$, but probably not $S_{4}$ and $S_{5}$ were involved in binding of substrates.

A recent study (103) shows that at least three subsites on the C-terminal side of the susceptible bond $\left(S_{1}^{\prime}-S_{3}^{\prime}\right)$ are important for hydrolysis. However, the rate of hydrolysis of all the peptides investigated is still several orders of magnitude lower than that fibrinogen. Thus, thrombin must have an unusual extended active site or the substrate must have a size large enough for a necessary folding to be accomplished, although, as pointed out above, the native conformation of fibrinogen is not mandatory.

\subsection{Papain}

Papain was the first enzyme in which SCHECHTER and BERGER (170) measured the size of an active site. This was estimated to extend over $25 \AA$ and to consist of 7 subsites $\left(S_{1}-S_{4}\right.$ and $\mathrm{S}_{1}^{\prime}-\mathrm{S}_{3}^{\prime}$ ) each of which occupied $3.5 \AA$. Subsites $S_{1}, S_{2}$ and $S_{1}^{\prime}$ showed very strong stereospecificity. Subsite $S_{2}$ was of particular interest since it showed a strong preference for the side-chains of Phe and Tyr (and to a smaller degree Leu). In this way these amino acid sidechains directed the hydrolysis to take place at the bond indicated by the arrow:

-Phe - $\mathrm{X} \perp \mathrm{Y}$ -

Thus, the specificity was not determined by the residue occupying $S_{\mathfrak{l}}$, the subsite usually responsible for strong side-chain specificity.

The specificity-directing effect of Phe and Tyr was abolished if $X$ was a valyl residue. Subsite $S_{1}$ apparently cannot accomodate the bulky isopropyl side-chain of Val. The consequence was that Val occupied $S_{2}$ and had the same effect as Phe. JOHANSEN and OtTesen (86) observed the same kind of specificity towards the B-chain of oxidized insulin, as did DESMAZEAUD (37) using glucagon as substrate. Streptococcal protease (201) hydrolyzed carboxymethylated B-chain of insulin and proteinase $\mathrm{A}_{2}$ from Actinidia chinensis (116) hydrolyzed the oxidized B-chain of insulin in a similar manner.

From binding studies involving peptide inhibitors BERGER and SCHECHTER (13) were able to determine the relative preference of subsites $S_{1}$ to $S_{4}$ for amino acid side-chains. $S_{2}$ was again shown to prefer hydrophobic side-chains. The same conclusion was reached with regard to $S_{1}$ but the binding here was not as strong as in $S_{2}$. BRUBACHER and BENDER (27) studied the influence of glycineamides of increasing chain length on the deacylation of trans-cinnamoyl papain and concluded that three subsites existed on the C-terminal end of the susceptible bond.

The conclusions arrived at from kinetic studies could be accounted for by model building using the known three-dimensional structure of papain (39). The picture arrived at in this way (13), however, did not agree with preliminary $X$-ray diffraction studies on papain-inhibitor complexes. It was pointed out that the "static" picture arrived at by X-ray analysis did not necessarily reflect the "dynamic conclusions based on kinetic studies.

\subsection{Microbial Proteases}

Pronase was shown by HARPER and BERGER (63) to have an active site consisting of five subsites. The active site of three neutral proteases 
obtained from Bacillus subtilis, Bacillus thermoproteolyticus, and Pseudomonas aeruginosa have been measured by MORIHARA and OKA (121). Both diastereoisomeric peptides and peptides in which the side-chains were varied were used, and it was concluded that the active sites in all three enzymes could be divided into at least six subsites $\left(S_{1}-S_{3}\right.$ and $\left.S_{1}^{\prime}-S_{3}^{\prime}\right)$.

A recent review by MoriHaRA (127) includes a number of other microbial proteases for which the size of the active site has been determined. This includes trypsin-like serine proteases from various strains of Streptomyces, the metal-chelator-sensitive alkaline protease from Pseudomonas aeruginosa, and acid proteases from Cladosporium, Aspergillus niger, Rhizobium chinensis, and Rhodotorula glutinis.

\section{CONCLUDING REMARKS}

The renewed interest in the study of hydrolysis of large peptides by proteolytic enzymes has provided much valuable information both about the size and the specificity of the active sites. While it is evident that active sites are rather large, extending from about 18 to $25 \AA$, it has not yet been proved that only one binding area or set of subsites participates in productive binding. In the subtilisins, for example, kinetic studies as well as certain chemical modification studies point to the existence of more than the single binding mode deduced from $\mathrm{X}$-ray crystallographic studies by KRAUT and coworkers. The discrepancies appear mainly to rest in the binding sites on the carboxyl-side of the susceptible bond, the region which is explored to the least extent by X-ray crystallography. Differences in this region between the two subtilisins are probably the reason for the quantitative differences in specificity observed between them.

The influence of secondary binding sites on the specificity of proteases varies. Trypsin, which is very side-chain specific in $S_{1}$, is not influenced with respect to specificity by changes in amino acid residues in other positions although the rate of hydrolysis of the specific bond changes with changes in adjacent amino acid residues. The reason for this is probably that the main contribution to binding is derived from the residue in $S_{1}$ while the proper alignment of the susceptible bond can be influenced by neighboring residues. If enough contacts can be made between enzyme and substrate, the dominating role of the specific side-chain in $S_{1}$ can be overcome as exemplified by the binding of pancreatic trypsin inhibitor to chymotrypsin in which case a lysyl residue occupies the hydrophobic $S_{1}$ subsite.

When the side-chain specificity becomes less dominant as in subtilisin and elastase, the influence of secondary binding sites on specificity will be more pronounced. Although the subtilisins have a hydrophobic $S_{1}$ subsite, strong binding at other subsites can easily outweigh the contribution of $S_{1}$ and therefore give rise to hydrolysis of a greater variety of bonds than observed with trypsin and chymotrypsin. Hydrolysis of the $\mathrm{Leu}_{15}-\mathrm{Tyr}_{16}$ bond in the $\mathrm{B}$ chain of oxidized insulin exemplifies this suggestion. Although Leu has a hydrophobic side-chain, hydrolysis of small ester substrates showed that the subtilisins were much more specific for Tyr than Leu, and hence one would expect $\mathrm{Tyr}_{16}$ bound in $\mathrm{S}_{1}$ rather than $\mathrm{Leu}_{15}$. However, the nice fit of the neighboring residues with the other subsites contributes so much binding energy that $\mathrm{Leu}_{15}$ become the preferred residue in $S_{1}$. The determination of the tertiary structure of the subtilisin BPN' complex with an inhibitor produced by Streptomyces (168) will be very interesting with respect to the residue bound in $S_{1}$. With the many more contacts expected in this case, the occurence of a Tyr, Trp or Phe in $S_{1}$ is even less certain than in the case of the B-chain.

Most of the work on the size of the active site in proteins has been performed by kinetic studies involving synthetic peptides. Precautions must be taken in interpreting the results. For example, the inclusion of various organic solvents in the reaction mixture with the purpose of keeping the substrate in solution might influence the subsites on the enzyme surface differently. Non-productive binding may not always be easy to detect and resolve, but may influence the results markedly as recently demonstrated by FASTREZ and FERSHT (44) for the hydrolysis of substituted anilides by chymotrypsin. The results obtained with synthetic substrates give 
information about the maximal binding area of an enzyme, but they do not identify the spatial positions of the individual subsites. However, this question can be answered from X-ray crystallographic studies or chemical modification studies. One draw-back in crystallographic studies is that they, for obvious reasons, are not performed with substrates, but with inhibitors (covalently bound, virtual substrates, or transition state analogs). Therefore information on the mode of binding on the carboxylic site of the susceptible bond can only be deduced from model building. Another draw-back is that the enzyme-substrate interactions deduced from Xray studies do not necessarily reflect the interactions in solution as demonstrated recently by VALLEE and coworker (89) for carboxypeptidase.

Chemical modification studies in conjunction with sequence determinations will in certain cases provide useful information about the secondary binding sites in enzymes. Thus, in the subtilisins a tyrosyl residue, $\mathrm{Tyr}_{104}$, has been shown to be part of an important subsite, and certain seryl/threonyl and carboxyl groups also take part in the binding of substrates. In the latter two cases, however, the position in the sequence has not been determined. While the identification of $\mathrm{Tyr}_{104}$ as part of subsite $\mathrm{S}_{4}$ in the subtilisins was in agreement with the crystallographic data, the suggested implication of $\operatorname{Ser}_{156}$ in subtilisin type Carlsberg as part of $S_{2}^{\prime}$ was not. This region is, however, less well defined in the crystallographic studies and the chemical modification studies, since an actual identification of the residue has not been made. Therefore it is premature to make any conclusion about the correct spatial arrangement of subsites $\mathbf{S}_{1}^{\prime}$ and $\mathbf{S}_{2}^{\prime}$ or the possibility of more than one productive binding mode. It should be pointed out, however, that kinetic studies using various inhibitors indicate that indeed more than one productive binding mode exists in the subtilisins.

One draw-back in the use of chemical modifications is that one cannot be sure of whether an already existing binding mode is modified or a new one is created. Another disadvantage is that it often is difficult to modify one residue selectively. This was possible with
$\mathrm{Tyr}_{104}$ in both subtilisins, but the evidence for participation of $\mathrm{Ser}_{156}$ in subtilisin type Carlsberg as part of a subsite is based on comparison with the spatial arrangement of the amino acid side-chains on the surface of subtilisin BPN' $^{\prime}$.

Apart from the results with the subtilisins, only a very limited number of data concerning the influence of chemical modifications on the secondary binding sites in proteolytic enzymes are available. When secondary binding sites were studied no chemical modification of the enzyme had been performed. When chemically modified enzymes were tested for activity, this had in most cases been donewith small synthetic substrates in which case only changes in or close to the catalytic site could be detected. It is the hope of the present author that future experiments with chemically modified enzymes will include assays on large peptide substrates or proteins as well as small synthetic substrates. Valuable new information about the interactions of large substrates with proteases can undoubtedly be obtained in this way, and much more information is certainly needed in order to get a more complete understanding of these interactions. The concerted effort of kinetic, crystallographic, and chemical studies is also necessary for a more complete picture of the interactions of proteolytic enzymes with their natural substrates, the proteins.

\section{SUMMARY}

Subtilisin type Carlsberg and subtilisin BPN' (which is identical to subtilisin type Novo) are two alkaline, extracellular proteases from $\mathrm{Ba}$ cillus subtilis and Bacillus amyloliquefacient, respectively. Both enzymes are well characterized: their amino acid sequences are determined, and the three-dimensional structure of subtilisin BPN' is known as well. The two enzymes are homologues, but differ in 85 positions in the amino acid sequences. All differences (except one) are localized on the surface of the molecule in the subtilisin BPN' enzyme, and it is therefore assumed that the three-dimensional structure of the two enzymes are nearly identical (section 1). 
Chemical modification of functional sidechains in amino acids is one of the methods available for the investigation of structure-function relationships of biologically active proteins. The subtilisins have been extensively investigated by means of this method, and the results are reviewed in section 2. Modification of lysine side-chains by f.ex. carbamylation (section 2.1.1) does not influence the enzymatic activity of the subtilisins while modification of the catalytically important serine and histidine side-chains (section 2.3.1 and 2.4) leads to total inactivation. A partially inactivated enzyme results from modification of a single methionine side-chain (section 2.5). Although the tryptophanyl side-chains are located near or on the surface of the enzyme molecule they are very unreactive (section 2.6).

Nitration or iodination of tyrosyl residues led to the surprising result that the enzymatic activity of subtilisin type Carlsberg towards certain polypeptide substrates (clupein, gelatine) was increased, while towards low molecular weight ester substrates it remained almost constant. Similar although not identical results were obtained with subtilisin BPN'. The nitrated tyrosines in subtilisin type Carlsberg were identified, and in addition it was shown that one tyrosyl side-chain, $\mathrm{Tyr}_{104}$, reacted much faster with the nitrating reagent tetranitromethane than any of the other tyrosyl residues. A comparison with the nitratable tyrosyl residues in subtilisin BPN' shows that tyrosyl residues which are located at identical positions in the sequences do not necessarily react identically with tetranitromethane.

The changes in the enzymatic properties of the subtilisins by nitration or iodination are explained by changes in the ability of the enzymes to bind substrate. In section 3.1 some definitions relating binding and hydrolysis of substrates are presented. Special emphasis is put on the concepts "subsites" and "secondary binding sites " developed by SCHECHTER and BERGER. These sites play an important role in the binding of polypeptides to proteolytic enzymes. Section 3.2 is a short description of the enzymatic mechanism. The substrate specificity of the subtilisins is the topic of section 3.3, where it is shown that the specificity demands determined by means of small synthetic ester substrates become more diffuse when polypeptides are hydrolyzed. This can be explained by the important role played by secondary binding sites.

Section 3.4.1 is a review of the systematic kinetic investigations in relation to secondary binding sites which have been conducted using synthetic polypeptides. It was concluded that the enzyme can "feel a least 6 amino acid residues around the bond which is hydrolyzed. This conclusion is, in part, confirmed by X-ray crystallographic investigations which are described in section 3.4.2. In this case 4 subsites on subtilisin $\mathrm{BPN}^{*}$, binding 4 residues on the $\mathrm{N}$ terminal side of the susceptible bond, have been identified.

The question of whether only substrates bound to these subsites, are hydrolyzed is discussed in section 3.4.3. Various kinetic as well as crystallographic investigations indicate that more than one productive binding is possible.

In section 3.4.4 the influence of chemical modification on the binding of large substrates to the subtilisins is discussed. The increase in activity of subtilisin type Carlsberg towards clupein and gelatine which resulted from nitration or iodination of $\mathrm{Tyr}_{104}$ is explained by modification of a secondary binding site in such a way that better binding is obtained for large substrates. However, small synthetic substrates are too small to include this binding site upon binding to the enzyme, and consequently the kinetics are not changed.

The very reactive $\mathrm{Tyr}_{104}$ appears to be part of one of the subsites localized by means of X-ray crystallography. Thus, in this case, good agreement is found between the two types of investigation. However, succinylation and glutarylation of the subtilisins, which also lead to enhancement of enzymatic activity, indicate that substrates can bind in a different productive mode.

Modification of certain carboxyl groups (section 2.7) seems also to influence the binding of peptide substrates. In this case a decrease, rather than an increase, in the enzymatic activity is observed.

At a degree of modification at which the residual activity of subtilisin $\mathrm{BPN}^{\prime}$ towards 
clupein is very low, the activity towards small synthetic ester substrates is hardly affected at all (section 3.4.4).

Nitration or iodination of $\mathrm{Tyr}_{104}$ lowers the $\mathrm{pK}$ of the phenolic group enough that it will carry a negative charge at the $\mathrm{pH}$ at which activity assays of the subtilisins normally are performed ( $\mathrm{pH}$ 8.0). While this negative charge is important for the binding of certain substrates, the main contribution to binding comes from hydrogen bonds and hydrophobic interactions. This has been verified by the author in experiments involving addition of high concentrations of neutral salts (section 3.4.5) and alcohols or guanidine hydrochloride (section 3.4.6). Addition of salts has the same enzymatic effect as nitration or iodination, and it is explained as a wsalting out effect". Alcohols and guanidine hydrochloride act as inhibitors, the effect of which is stronger on large substrates, an observation which is in agreement with binding of alcohol to secondary binding sites.

Section 4 is a discussion of a series of proteases which have been investigated with respect to secondary binding sites. Of special interest are chymotrypsin, trypsin and elastase since the enzymatic mechanism of these enzymes are identical to the one found in the subtilisins. It is therefore of interest to investigate if this similarity also extends to binding of large substrates.

Our knowledge about binding of large substrates is summarized in section 5. For several proteases, it is known how many amino acid residues of a peptide chain can be bound, but it is still uncertain how large an area of the enzyme surface takes part in the binding process. An evaluation of the advantages and disadvantages of the three methods - X-ray crystallography, kinetic studies with synthetic peptides and chemical modifications - which have been described for determination of secondary binding sites in proteases has been attempted. None of the methods alone can give all the answers, but by using them concomitantly we might get a more complete picture of substrate binding to proteases.

\section{ACKNOWLEDGEMENTS}

The author wishes to express his appreciation to Professor Martin OTTESEN for his penetrating criticism of ideas and conclusions and his never failing interest in the experimental work which forms the basis of this review article. I also want to thank all my colleagues at the department who over the years have been of great help both in practical matters and as moral support. The linguistic correction made by Dr. HENRY ABRASH are warmly acknowledged.

\section{REFERENCES}

1. Abramowitz, N., I. Schechter and A. Berger: On the size of the active site in proteases II. Carboxypeptidase-A. Biochem. Biophys. Res. Commun. 29: 862-867 (1967)

2. Abramowitz-KURN, N. and 1. SChEChter: Mapping the active site of carboxypeptidase A; a proposed scheme for substrate binding at the active site. Israel J. Chem. 12: 543-555 (1947)

3. ADAMS, K. R.: Investigations on the chemical reactivity of the carboxyl groups in subtilisin type Novo. Compt. Rend. Trav. Lab. Carlsberg 38: 481-498 (1972)

4. Alden, R. A., C. S. Wright, F. C. Westall and J. KRAUT: Subtilisin BPN': Tertiary structure and inhibitor binding. Abstract in: $P$. Desnuelle, H. Neurath and M. Ottesen, eds., Structure-Function Relationships of Proteolytic Enzymes, p. 173. Munksgaard (Copenhagen) 1970

5. ANDO, T. and K. Suzukl: The amino acid sequence of the third component of clupein. Biochim. Biophys. Acta 140:375-377 (1967)

6. Andreatta, R. H., R. K. H. Liem and H. A. SCHERAGA: Mechanism of action of thrombin on fibrinogen. I. Synthesis of fibrinogen-like peptides, and their proteolysis by thrombin and trypsin. Proc. Natl. Acad. Sci. U. S. 68: 253-256 (1971)

7. Aoyagi, T., S. Miyata, M. Nanbo, F. Kojima, M. Matsuzaki, M. Ishizuka, T. Takeuchi and H. UMEZAVA: Biological activities of leupeptins. J. Antibiotics (Tokyo) 22: 558-568 (1969)

8. Atlas, D., S. Levit, I. SChechter and A. Berger: On the active site of elastase: Partial mapping by means of specific peptide substrates. FEBS Lett. 11:281-283 (1970)

9. Atlas, D. and A. BerGer: On the specificity of elastase. Hydrolysis of peptide p-nitrobenzyl esters. Biochemistry 11: 4719-4723 (1972)

10. Bailey, K. and F. R. BetTelheim: The nature of the fibrinogen-thrombin reaction. Brit. Med. Bull. 11: 50 (1955) 
11. Barel, A. O. and A. N. Glazer: Comparative studies of the enzymatic properties of Novo and Carlsberg subtilisins. J. Biol. Chem. 243: 13441348 (1968)

12. Bender, M. L. and F. J. Kedzy: Mechanism of action of proteolytic enzymes. Ann. Rev. Biochem. 34: 49-76 (1965)

13. Berger, A. and I. Schechter: Mapping the active site of papain with the aid of peptide substrates and inhibitors. Phil. Trans. Roy. Soc. Lond, B. 257: 249-264 (1970)

14. Bettelheim, F. R.: The clotting of fibrinogen II. Fractionation of peptide material liberated. Biochim. Biophys. Acta 19: 121-130 (1956)

15. Birktoft, J. J., D. M. Matthews, T. A. Poulus and J. KRAUT: A crystallographic view of the serine protease mechanism. Abstract: V. Linderstrøm-Lang Conference, Vingsted Denmark (1975)

16. Bisby, R. H., R, B. Cundall, G. E. Adams and J. L. RedPaTH: Selective free radial reactions with proteins and enzymes. The inactivation of subtilisin Carlsberg and subtilisin Novo, J. Chem. Soc. Faraday Trans. 1, 70: 2210-2218 (1974)

17. Bцомвӓ., B., М. Blombäck, P. Edman and B. Hessel: Human fibrino-peptides. Isolation, characterization and structure. Biochim. Biophys. Acta 115: 371-396 (1966)

18. BLOMBÄK, B. and A. VESTERMARK: Isolation of fibrino-peptides by chromatography. Arkiv Kemi 12: 173-182 (1958)

19. Blow, D. M., J. J. Birktoft and B. S. Hartley: Role of a buried acid group in the mechanism of action of chymotrypsin. Nature 221: 337-340 (1969)

20. Boesel, R.W. and F. H. Carpenter Crosslinking during the nitration of bovine insulin with tetranitromethane. Biochem. Biophys. Res. Commun. 38: 678-682 (1970)

21. Bosshard, H. R.: Kinetic evidence for two different productive substrate binding sites in subtilisins. FEBS Lett. 30:105-110 (1973)

22. Bosshard, H. R, and A. Berger: The topographical differences in the active site region of a-chymotrypsin, subtilisin Novo, and subtilisin Carlsberg. Mapping the aromatic binding site by inhibitors (virtual substrates). Biochemistry 13: 266-277 (1974)

23. Bosshard, H. R.: Binding of D-amino acid residues to the aromatic binding sites of $a-$ chymotrypsin, subtilisin Novo and subtilisin Carlsberg. Israel J. Chem. 12:495-504 (1974)

24. Boyer, H. W. and B. C. Carlton: Production of two proteolytic enzymes by a transformable strain of Bacillus subtilis. Arch. Biochem. Biophys. 128: 442-455 (1968)

25. BRETZEL, G.: Versuche zur spaltung von clupein durch pepsin. Hoppe-Seyler's Z. Physiol. Chem. 354: 1017-1019 (1973)
26. Brown, W. E. and F. Wold: Alkyl isocyanates as active-site-specific reagents for serine proteases. Identification of the active-site serine as the site of reaction. Biochemistry 12: 835-840 (1973)

27. BRubacher, L. J. and M. L. Bender: Reaction of trans-cinnamoyl papain with a series of polyglycinamides of varying chain-length. Biochem. Biophys. Res. Commun. 27: 176-182 (1967)

28. Bruice, T. C., M. J. Gregory and S. L. WALTERS: Reactions of tetranitromethane. I. Kinetics and mechanism of nitration of phenols by tetranitromethane. J. Amer. Chem. Soc. 90 : 1612-1619 (1968)

29. Brutlag, D., M. R. Atkinson, P. Setlow and A. KoRNBERG: An active fragment of DNA polymerase produced by proteolytic cleavage. Biochem. Biophys. Res. Commun. 37: 982 (1969)

30. Chou, J. Y. and M. F. Singer: The effect of chain length on the phosphorolysis of oligonucleotides by polynucleotide phosphorylase. J. Biol. Chem. 245: 1005-1011 (1970)

31. Coggins, J. R., W. Kray and E. Shaw: Affinity labelling of proteinases with tryptic specificity by peptides with C-terminal lysine chloromethyl ketone. Biochem. J. 138: 579-585 (1974)

32. CoHEN, L. A.: Group-specific reagents in protein chemistry. Ann. Rev. Biochem. 37: 695 725 (1968)

33. Cohen, W. and B. F. Erlanger: Studies on the reactivation of diethylphosphorylchymotrypsin. J. Amer. Chem. Soc. 82: 3928-3934 (1960)

34. Cohen, W., M. Lache and B. F. Erlanger: The reactivation of diethylphosphoryltrypsin. Biochemistry 1: 686-693 (1962)

35. Cuatrecasas, P., M. Wilchek and C. B. ANFINSEN: Staphylococcal nuclease: Size and specificity of the active site. Science 162: 1491 1493 (1968)

36. Davies, R. C., D. S. Aauld and B. L. Vallee The effect of modifiers on the hydrolysis of esters and peptides by carboxypeptidase A. Biochem. Biophys. Res. Commun. 31: 628-633 (1968)

37. Desmazeaud, M. J.: Contribution a l'étude de la spécificité de la papaine: hydrolyse du glucagon. Biochimie 54: 1109-1114(1972)

38. Doscher, M.S. and C. H.W. HiRs: The heterogeneity of bovine pancreatic ribonuclease S. Biochemistry 6: 304-311 (1967)

39. Drenth, J., J. N. Jansonius, R. Koekoek, H. M. SWEN and B. G. WOLTERS: Structure of papain. Nature, London 218: 929-932 (1968)

40. Drenth, J., W. G. J. Hol, J. N. JAnsonius and R. Koekoek: Subtilisin Novo. The three-dimensional structures of subtilisin BPN' and subtilisin Novo. Cold Spring Harbor Symp. Quant. Biol. 36: 107-116 (1971)

41. Drenth, J., W. G. J. Hol; J. N. Jansonjus and R. Koekoek: Subtilisin Novo. The three dimen- 
sional structure and its comparison with subtilisin BPN'. Eur. J. Biochem. 26: 177-181 (1972)

42. Erlanger, B. F. and W. Cohen: Specific inactivation of chymotrypsin by diphenylcarbamyl chloride. J. Amer. Chem. Soc. 85: 348-349 (1963)

43. Erlanger, B. F. and F. Edel: The utilization of a specific chromogenic inactivator in an wall or none" assay for chymotrypsin. Biochemistry 3 : 346-349 (1964)

44. Fastrez, J. and A. R. Fersht: Mechanism of chymotrypsin. Structure, reactivity, and nonproductive binding relationships. Biochemistry 12: $1067-1074(1973)$

45. Fischer, E. and P. Bergell: Ueber die Derivative einiger Dipeptide und ihr Ferhalten gegen Pancreasfermente. Berichte 36: 25922608 (1903)

46. Fruton, J. S.: The specificity and mechanism of pepsin action. Adv. Enzymol. 33: 401-443 (1970)

47. Fruton, J. S.: Pepsin. In: P. D. Boyer, ed., The enzymes, Vol. III, pp. 119-164. Academic Press (New York and London) 1971

48. Gertler, A.: Selective, reversible loss of elastolytic activity of elastase and subtilisin resulting from electrostatic changes due to maleylation. Eur. J. Biochem. 23: 36-40 (1971)

49. Gerwin, B. I., W. H. Stein and S. Moore: On the specificity of streptococcal proteinase. J. Biol. Chem. 241: 3331 -3339 (1966)

50. GLADNER. J. A. in "Fibrinogen “, K. Laki editor, Marcel Dekker Inc., N.Y., 1968 pg. 87

51. Gladner, J. A., J. E. Folk, K. Laki and W. R. CARRolL: Thrombin-induced formation of cofibrin. J. Biol. Chem. 234: 62-66 (1959)

52. Glazer, A. N.: Transesterification reactions catalyzed by subtilisins. J. Biol. Chem. 241: 635$638(1966)$

53. Glazer, A. N.: Esteratic reactions catalyzed by subtilisins. J. Biol. Chem. 242: 433-436 (1967)

54. Glazer, A. N.: The time-dependent specific interactions of 4-(4'-aminophenylazo) phenylarsonic acid with subtilisins. Proc. Natl. Acad. Sci. U.S. 59: 996-1002 (1968)

55. GlAZER, A. N.: Inhibition of "serine" esterases by phenylarsonic acids. J. Biol. Chem. 243: 3693-3701 (1968)

56. Gounaris, A. and M. Ottesen: Some properties of succinylated subtilopeptidase. Compt. Rend. Trav. Lab. Carlsberg 35: 37-62 (1965)

57. GraAE, J.: Esterase activity shown by subtilisin, a proteolytic enzyme from Bacillus subtilis. Acta Chem. Scand. 8: 356-357 (1954)

58. GROss, E. and B. WITKOP: The heterogeneity of the $S$ peptide of bovine pancreatic ribonuclease A. Biochemistry 6: 745-748 (1967)

59. GUNTElberg, A.V. and M. OTTESEN: Purification of the proteolytic enzyme from Bacillus subtilis. Compt. Rend. Trav. Lab. Carlsberg, Ser. Chim. 29: 36-48 (1954)

60. Habeeb, A. F. S. A.: Preparation of enzymati- cally active, water-insoluble derivatives of trypsin. Arch. Biochem. Biophys. 119: 264-268 (1967)

61. Hageman, J. H. and B. C. Carlton: An enzymatic and immunological comparison of two proteases from a transformable Bacillus subtilis with the "subtilisins". Arch. Biochem. Biophys. 139: 67.79 (1970)

62. Hagihara, B., H. Matsubara, M. Nakal and K. OKUnuKI: Crystalline bacterial proteinase. I. Preparation of crystalline proteinase of Bacillus subtilis. J. Biochem. (Tokyo) 45: 185-194 (1958)

63. Harper, E. and A. Berger: On the size of the active site in proteases: pronase. Biochem. Biophys. Res. Commun. 46: 1956-1960 (1972)

64. HartLey, B. S.: Proteolytic enzymes. Ann. Rev. Biochem. 29: 45-72 (1960)

65. Hashimori, Y., H. Horinishi, H. Kurihara and K. Shibata: States of amino acid residues in proteins. V. Different reactivities with $\mathrm{H}_{2} \mathrm{O}_{2}$ of tryptophan residues in lysozyme, proteinases and zymogen. Biochim. Biophys. Acta 93: 346360 (1964)

66. Haugaard, E. S. and N. Haugaard: Degradation of crystalline insulin by subtilisin (a proteinase from B. subtilis). Compt. Rend. Trav. Lab. Carlsberg, Ser. Chim. 29: 350-365 (1955)

67. Hein, G. E. and C. NiemanN: Steric course and specificity of a-chymotrypsin-catalyzed reactions II. J. Amer. Chem. Soc. 84: 4495-4503 (1962)

68. HensCHEN, A.: S-sulfo-derivatives from fibrinogen and fibrin: preparations and general properties. Arkiv Kemi 22: 1-28 (1963)

69. Herskovits, T. T. and H. H. Fuchs: Solvent perturbation of the tyrosyl and tryptophanyl residues in subtilisin BPN'. Biochim. Biophys. Acta 263: 468-476 (1972)

70. HILL, R. D.: The nature of the rennin-sensitive bond in casein and its possible relation to sensitive bonds in other proteins. Biochem. Biophys. Res. Commun. 33: 659-663 (1968)

71. Hiromi, K., Y. NitTa, C. Numata and S. Ono: Subsite affinities of glucoamylase: examination of the validity of the subsite theory. Biochim. Biophys. Acta 302: 362-375 (1973)

72. Hoare, D. G. and D. E. Koshland, Jr.: A method for the quantitative modification and estimation of carboxylic acid groups in proteins. J. Biol. Chem. 242: 2447-2453 (1967)

73. Hollands, T. R., I. M. Voynick and J.S. FRUTON: Action of pepsin on cationic synthetic substrates. Biochemistry 8: 575-585 (1969)

74. Horton, H. R. and D. E. Koshland, Jr.: A highly reactive colored reagent with selectivity for the tryptophan residue in proteins. 2-hydroxy-5-nitrobenzyl bromide. J. Amer. Chem. Soc. 87: 1126-1132(1965)

75. Huber, R., D. Kukla, A. Ruhlmann and W. SteigemanN: Pancreatic trypsin inhibitor (Kunitz). Cold Spring Harbor Symp. Quant. 
Biol. 36: 141-150 (1972)

76. HUBER, R.: Interaction between chymotrypsin (trypsin) and the pancreatic trypsin inhibitor (Kallikrein inactivator). In: R. JAENICKE and E. Helmreich, eds., Protein-Protein Interactions. Springer Verlag. 1972

77. Hughes, Jr., W. L. and R. Straessle: Preparation and properties of serum and plasma proteins. XXIV. lodination of human serum albumin. J. Amer. Chem. Soc. 72: 452-457 (1950)

78. Humphreys, R. E. and J.S. Fruton: The substrate binding site of pepsin. Proc. Natl. Acad. Sci. U.S. 59: 519-525 (1968)

79. Hunkapiller, M. W., S. H. Smallcombe, D. R. WhITAKER and J. H. Richards: Carbon nuclear magnetic resonance studies of the histidine residue in $\alpha$-lytic protease. Implications for the catalytic mechanism of serine proteases. Biochemistry 12: 4732-4743 (1973)

80. Hunt, J. A. and M. OTtesen: A comparison of three proteinases from various strains of Bacillus subtilis. Biochim. Biophys. Acta 48: 411-412 (1961)

81. IzumiYa, N. and H. Uchio: Action of trypsin on synthetic substrates. I. Action of trypsin on mono-, di-, tri-, and tetra-glycyl-L-lysineamide. J. Biochem. (Tokyo) 46: 645-652 (1959)

82. IZumeya, N. and H. Uchio: Action of carboxypeptidase on synthetic substrates. I. Action of carboxypeptidase on mono-, di-, tri-, tetra-, and penta-glycyl-L-tyrosine. J. Biochem. (Tokyo) 46: 235-245 (1959)

83. Johansen, G. and M. Ottesen: The amino acid compositions of three proteinases from Bacillus subtilis. Compt. Rend. Trav. Lab. Carlsberg 34: 199-210 (1964)

84. Johansen, J. T., M. Ottesen and I. Svendsen: Chemical derivatives of subtilisin Carlsberg with increased proteolytic activity. Biochim. Biophys. Acta 139: 211-214 (1967)

85. Johansen, J. T., M. Ottesen, I. Svendsen and G. WYBRANDT: The degradation of the B-chain of oxidized insulin by two subtilisins and their succinylated and $\mathrm{N}$-carbamylated derivatives. Compt. Rend. Trav. Lab. Carlsberg 36: 365-384 (1968)

86. Johansen, J. T. and M. Ottesen: The proteolytic degradation of the B-chain of oxidized insulin by papain, chymopapain and papaya peptidase. Compt. Rend. Trav. Lab. Carlsberg 36: 265-283 (1968)

87. Johansen, J. T., R. W. A. Oliver and I. SVENDSEN: Some spectral and kinetic properties of the acyl enzymes of native and chemically modified subtilisin type Carlsberg and type Novo. Compt. Rend. Trav. Lab. Carlsberg 37: 87-105 (1969)

88. JOHANSEN, J. T.: Chemical derivatives of subtilisin with modified proteolytic activities. II. Succinyl- and glutarylsubtilisin type Carlsberg. Compt. Rend. Trav. Lab. Carlsberg 37: 145-177
(1970)

89. JOHANSEN, J. T. and B. L. VAlleE: Environment and conformation dependent sensitivity of the arsanilazotyrosine-248 carboxypeptidase A chromophore. Biochemistry 14: 649-660 (1975)

90. Johnson, L. N. and D. C. PhilLips: Structure of some crystalline lysozyme-inhibitor complexes determined by X-ray analysis at $6 \AA$ resolution. Nature 206: 761-763 (1965)

91. Kahana, L. and Y. Shalitin: Salt effects on the properties of a-chymotrypsin. I. Effects on the enzymic activity of chymotrypsin. Israel $\mathrm{J}$. Chem. 12:573-589 (1974)

92. KeAY, L. and P. W. Moser: Differentiation of alkaline proteases from bacillus species. Biochem. Biophys. Res. Commun. 34: 600-604 (1969)

93. Keay, L., P. W. Moser and B. S. WiLdi: Proteases of the genus bacillus II. Alkaline proteases. Biotechnol. Bioeng. 12: 213-249 (1970)

94. Klee, W. A. and R. A. Streaty: Studies on the conformation and proteolytic susceptibility of rat and bovine pancreatic ribonucleases. J. Biol. Chem. 245: 1227-1232 (1970)

95. Klenow, H. and I. Henningsen: Selective elimination of the exonuclease activity of the DNA polymerase from Escherichia coli B by limited proteolysis. Proc. Natl. Acad. Sci. U.S. 65: 168-175 (1970)

96. Koehler, K. A and G. E. Lienhard: 2-phenylethaneboronic acid, a possible transition-state analog for chymotrypsin. Biochemistry 10:24772483 (1971)

97. Koshland, D. E., Jr. and K. E. Neet: The catalytic and regulatory properties of enzymes. Ann. Rev. Biochem. 37: 359-410 (1968)

98. Kraut, J., J. D. Robertus, J. J. Birktoft, R. A. AldEN, P. E. WILCOX and J. C. POWERS: The aromatic substrate site in subtilisin BPN' $^{\prime}$ and its resemblance to chymotrypsin. Cold Spring Harbor Symp. Quant. Biol, 36: 117-123 (1971)

99. Kurachi, K., J. C. Powers and P. E. Wilcox: Kinetics of the reaction of chymotrypsin $A_{0}$ with peptide chloromethyl ketones in relation to its subsite specificity. Biochemistry 12: 771-777 (1973)

100. Kurihara, M., F. S. Markland and E. L. SMITH: Subtilisin amylosaccariticus. J. Biol. Chem. 247: 5619-5631 (1972)

101. LatT, S. A., D. S. Auld and B. L. ValleE: Distance measurements at the active site of carboxypeptidase A during catalysis. Biochemistry 11: 3015-3022 (1972)

102. Liem, R. K. H., R. H. Andreatta and H. A. SCHERAGA: Mechanism of action of thrombin on fibrinogen. II. Kinetics of hydrolysis of fibrinogen-like peptides by thrombin and trypsin. Arch. Biochem. Biophys. 147: 201-213 (1971)

103. Liem, R. K. H. and H. A. Scheraga: Mechanism of action of thrombin on fibrinogen. 
IV. Further mapping of the active sites of thrombin and trypsin. Arch. Biochem. Biophys. 160: 333-339 (1974)

104. Linderstrøm-Lang, K. and M. Ottesen: A new protein from ovalbumin. Nature 159: 807808 (1947)

105. LinderstrøM-Lang, $K$. and M. Ottesen: Formation of plakalbumin from ovalbumin. Compt. Rend. Trav. Lab. Carlsberg, Ser. Chim. 26: 403-442 (1949)

106. LindQuist, R. N. and C. TerRy: Inhibition of subtilisin by boronic acids, potential analogs of tetrahedral reaction intermediate. Arch. Biochem. Biophys. 160: 135-144 (1974)

107. LIU, Y. P. and R. E. Handschumacher: Nitroasparaginase. Subunit crosslinkage and altered substrate specificity. J. Biol. Chem. 247: 66-69 (1972)

108. Loudon, G. M. and D. E. Koshland, Jr.: The chemistry of a reporter group: 2-hydroxy-5. nitrobenzyl bromide. J. Biol. Chem. 245: 22472254 (1970)

109. Markl.AND, F. S. and E. L. Smith: Subtilisin

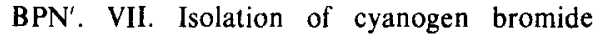
peptides and the complete amino acid sequence. J. Biol. Chem. 242: 5198-5211 (1967)

110. Markland, F. S., E. Shaw and E. L. Smith: Identification of histidine 64 in the active site of subtilisin. Pro. Natl. Acad. Sci. U. S. 61:14401447 (1968)

111. MaRKLAND, F. S.: Exposed tyrosine residues of subtilisin Novo. Fed. Proc. 28: 877 (1969)

112. Markland, F. S.: Phenolic hydroxyl ionization in two subtilisins. J. Biol. Chem. 244: 694-700 (1969)

113. Markland, F. S., Jr. and E. L. Smith: Subtilisins: Primary structure, chemical and physical properties. In: P. D. BOYER, ed., The Enzymes, Vol. III, pp. 561-608, 3.edition. Academic Press 1971.

114. Matsubara, H. and S. Nishimura: Crystalline bacterial proteinase. V. On the reaction with diisopropyl fluorophosphate. J. Biochem. (Tokyo) 45: 503-510 (1958)

115. Matthews, D. A., R. A. Alden, J. J. Birktoft, S. T. Freer and J. KraUt: X-ray crystallographic study of boronic acid adducts with subtilisin $\mathrm{BPN}^{\prime}$ (Novo). A model for the catalytic transition state. J. Biol. Chem. 250: 7120-7126 (1975)

116. McDowall, M. A.: The action of proteinase $A_{2}$ from Actinidia chinensis on the B-chain of oxidized insulin. Biochim. Biophys. Acta 293: 226231 (1973)

117. Medzihradszky, K., I. M. Voynick, H. Medzlhradszky-Shweiger and J. S. Fruton: Effect of secondary enzyme-substrate interactions on the cleavage of synthetic peptides by pepsin. Biochemistry 9: 1154-1162 (1970)

118. MeEdon, B.: The action of subtilisin on crystalline pork insulin. Compt. Rend. Trav.
Lab. Carlsberg, Ser. Chim. 29: 403-416 (1955)

119. Myers II, B. and A. N. Glazer: Spectroscopic studies of the exposure of tyrosine residues in proteins with special reference to the subtilisins. J. Biol. Chem. 246: 412-419 (1971)

120. Millet, J.: Charactérisation de deux endopeptidases excrétées par $B$. subtilis Marburg au cours de la sporulation. Bull. Soc. Chim. Biol. $51: 457-469$ (1969)

121. Morihara, K. and T. OKa: The complex active sites of bacterial neutral proteases in relation to their specificities. Biochem. Biophys. Res. Commun. 30: 625-630 (1968)

122. Morihara, K. and H. TsuzukI: Comparison of the specificities of various serine proteinases from microorganisms. Arch. Biochem. Biophys. 129: 620-634 (1969)

123. Morihara, K, T. Oka and H. Tsuzuki: Comparison of $\alpha$-chymotrypsin and subtilisin BPN': Size and specificity of the active site. Biochem. Biophys. Res. Commun. 35: 210-214 (1969)

124. Morihara, K, T. OKa and H. Tsuzuki: Subtilisin BPN': Kinetic study with oligopeptides. Arch. Biochem. Biophys. 138: 515-525 (1970)

125. Morihara, K. and T. Oka: Subtilisin BPN': Inactivation by chloromethyl ketone derivatives of peptide substrates. Arch. Biochem. Biophys. 138: 526-531 (1970)

126. Morihara, K., H. Tsuzuki and T. Oka: Comparison of various types of subtilisins: Size and properties of the active site. Biochem. Biophys. Res. Commun. 42: 1000-1006 (1971)

127. Morihara, K.: Comparative specificity of microbial proteinases. Adv. Enzymology 41: 179-243 (1974)

128. MorrisetT, J. D. and C. A. Broomfield: A comparative study of spinlabeled serine enzymes: Acetylcholinesterase, trypsin, $\alpha$-chymotrypsin, elastase, and subtilisin. J. Biol. Chem. 247: 7224-7231 (1972)

129. Neet, K. E., A. Nanci and D. E. Koshland, Jr.: Properties of thiolsubtilisin. J. Biol. Chem. 243: 6392-6401 (1968)

130. Neil, G. L., C. Niemann, and G. E. Hein: Structural specificity of a-chymotrypsin: polypeptide substrates. Nature 210: 903-907 (1966)

131. Noller, H. F. and S. A. Bernhard: Isolation and structural determination of chromophoric acyl peptides from subtilisin enzymes. Biochemistry 4: 1118-1126 (1965)

132. Ogata, K., M. Ottesen, and I. Svendsen: Preparation of water-insoluble enzymatically active derivatives of subtilisin type Novo by crosslinking with glutaraldehyde. Biochem. Biophys. Acta 159: 403-405 (1968)

133. Ohtsuki, K., C. L. Liu and H. Hatano: Inactivation of subtilisin $\mathrm{BPN}^{\prime}$ by $\mathrm{N}-\mathrm{Bro}-$ mosuccinimide. J. Biochem. (Tokyo) 66: 863865 (1969)

134. OKa, T., and K. Morimara: Specificity of 
pepsin: size and property of the active site. FEBS Letters 10: 222-224 (1970)

135. Okunuki, K., H. Matsubara, S. Nishimura, and B. Hagihara: Specificity of crystalline bacterial proteinase. J. Biochem. (Tokyo) 43: 857-866 (1956)

136. Olaitan, S. A., R. J. Delange, and E. L. SMITH: The structure of subtilisin Novo. J. Biol. Chem. 243: 5296-5301 (1968)

137. Oosterbann, R. A. and J. A. Cohen: The active site of esterases. In: T. W. GoodwIN, J. I. HARRIS and B. S. HARTLEY, eds., Structure and activity of enzymes, pg. 87. Academic Press 1971.

138. Ottesen, $M$. and C. G. Schellman: The preparation of crystalline diisopropyl fluorophosphate derivative of subtilisin and the determination of its free amino end group. Compt. Rend. Trav. Lab. Carlsberg, Ser. Chim. 30: 157-166 (1957)

139. OTTESEN, M.: The transformation of ovalbumin into plakalbumin. A case of limited proteolysis. Compt. Rend. Trav. Lab. Carlsberg, Ser. Chim. 30: $211-270(1958)$

140. Ottesen, M. and B. Ostergaard: The limited proteolysis of native ovalbumin by Bacillus subtilis proteinases. Compt. Rend. Trav. Lab. Carlsberg 34: 187-198 (1964)

141. Ottesen, M., J. T. Johansen and I. Svendsen: Subtilisin: Stability properties and secondary binding sites. In: P. Desnuelle, H. Neurath and $M$. OTtEsEN, eds., Structure-function relationships of proteolytic enzymes, pp. 175186. Munksgård (Copenhagen) 1970.

142. Ottesen, M. and I. Svendsen: The subtilisins. In: G. E. PERLManN and L. Lorand, eds., Methods in Enzymology, Vol. 19, pp. 199-215. Academic Press 1970.

143. Ottesen, M. and 1. Svendsen: The effects of salts on the enzymatic activity of subtilisin type Carlsberg and subtilisin type Novo. Compt. Rend. Trav. Lab. Carisberg 38: 369-383 (1971)

144. Ottesen, M. and G. Ralston: The ionization behaviour of subtilisin type Novo. Compt. Rend. Trav. Lab. Carlsberg 38: 457-479 (1972)

145. Ozawa, M.: Bridging reagent for proteins. I. J. Biochem. (Tokyo) 62: 419-423 (1967)

146. PHILIPP, M. and M. L. BeNDER: Inhibition of serine proteases by arylboronic acids. Proc. Natl. Acad. Sci. U. S. 68: 478-480 (1971)

147. Polgar, L. and M. L. Bender: The reactivity of thiol-subtilisin, an enzyme containing a synthetic functional group: Biochemistry $6: 610-620$ (1967)

148. Polgar, L.: Conversion of the serine residue at the active site of alcalase to a cysteine side-chain. Acta Biochim. Biophys. Acad. Sci. Hung. 3: 397 (1968)

149. Potts, J. T., A. Berger, J. Cooke and C. B. ANFINSEN: A reinvestigation of the sequence of residues 11 to 18 in bovine pancreatic ribo- nuclease. J. Biol. Chem. 237: 1851-1855 (1962)

150. Quiocho, F. A. and F. M. RICHARDS: Intermolecular cross linking of a protein in the crystalline state: carboxypeptidase-A: Proc. Natl. Acad. Sci. U. S. 52: 833-839 (1964)

151. RALSTON, G.: On the alcohol inhibition of enzyme activity. Compt. Rend. Trav. Lab. Carisberg 39: 25-31 (1972)

152. Raymond, M. N., J. Garnier, and E. Bricas: Studies on the specificity of chymosin (rennin). I. Kinetic parameters of the hydrolysis of synthetic olipopeptide substrates. Biochimie 54: 145-154 (1972)

153. Richards, F. M. and P. J. Vithayathil: The preparation of subtilisin-modified ribonuclease and the separation of the peptide and protein components. J. Biol. Chem. 234: 1459-1465 (1959)

154. Riordan, J. F. and B. L. ValleE: Acetylcarboxypeptidase. Biochemistry 2: 1460-1467 (1963)

155. Riordan, J. F. and B. L. VAlleE: Succinylcarboxypeptidase. Biochemistry 3: $1768-1774$ (1964)

156. Riordan, J. F., W. E. C. WACKer, and B. L. VALLEE; N-acetylimidazole: A reagent for determination of "free tyrosyl residues of proteins. Biochemistry 4: 1758-1765 (1965)

157. Riordan, J. F., M. Sokolovsky, and B. L. VALLEE: Environmentally sensitive tyrosyl residues, Nitration with tetranitromethane. Biochemistry 6: 358-361 (1967)

158. Riordan, J. F. and D. M. Livingston: Conformational and activity changes on subtilisin cleavage of carboxypeptidase A. Biochem. Biophys. Res. Commun. 44: 695-701 (1971)

159. Robertus, J. D., R. A. Alden, J. J. Birktoft, J. Kraut, J. C. Powers, and P. E. Wilcox: An Xray crystallographic study of the binding of peptide chloromethyl ketone inhibitors to subtilisin BPN'. Biochemistry 11: 2439-2449 (1972)

160. Robertus, J. D., J. Kraut, R. A. Alden, and J. J. Birktoft: Subtilisin; a stereochemical mechanism involving transition-state stabilisation. Biochemistry 11:4293-4303 (1972)

161. Robillard, G. T., J. C. Powers, and P. E. WILCOX: A chemical and crystallographic study of carbamyl-chymotrypsin A. Biochemistry 11: 1773-1784 (1972)

162. Robyt, J. and D. French: Action pattern and specificity of an amylase from Bacillus subtilis. Arch. Biochem. Biophys. 100:451-467 (1963)

163. RobyT, J. F. and D. French: The action pattern of porcine pancreatic a-amylase in relationship to the substrate binding site of the enzyme. J. Biol. Chem. 245: 3917-3927 (1970)

164. Rupley, J. A.: The binding and cleavage by lysozyme of $\mathrm{N}$-acetylglucosamine oligosaccarides. Proc. Royal Soc. Ser. B, Biol. Sci. 167: 416-428 (1967)

165. SAChdev, G. P. and J. S. Fruton: Pyridyl esters of peptides as synthetic substrates of pepsin. Biochemistry 8: 4231-4246 (1969) 
166. SAChdev, G. P., M. A. Johnston, and J. S. FRUTON: Fluorescence studies on the interaction of pepsin with its substrates. Biochemistry 11: 1080-1086 (1972)

167. Sanger, F, and D. C. Shaw: Amino-acid sequence about the reactive serine of a proteolytic enzyme from Bacillus subtilis. Nature 187: 872 $873(1960)$

168. Satow, Y., Y. Mitsul, Y. Iitaka, S. Murao, and S. SATO: Crystallization and preliminary $X$-ray investigation of a new alkaline protease inhibitor and its complex with subtilisin $\mathrm{BPN}^{\prime}$. J. Mol. Biol. 75: 745-746 (1973)

169. Schattenkerk, C., I. Holtkamp, J. G. M. Hessing, K. E. T. Kerling, and E. Havinga: Studies on polypeptides: XIII. Relation between structure and capacity to function as rennin substrate. Rec.trav.chim. 90: 1320-1322 (1971)

170. Schechter, I. and A. Berger: On the size of the active site in proteases. I. Papain. Biochem. Biophys. Res. Commun. 27: 157-162 (1967)

171. Schechter, 1. and A. Berger: The hydrolysis of diastereoisomers of alanine peptides by carboxypeptidase A and leucine aminopeptidase. Biochemistry 5: 3371-3375 (1966)

172. SChlessinger, J., R. S. Roche and I. Z. SteinberG: A study of subtilisin types Novo and Carlsberg by circular polarization of fluorescence. Biochemistry 14: 255-262 (1975)

173. Segal, D. M., J. C. Powers, G. H. Cohen, D. R. DAviEs, and P. E. Wilcox: Substrate binding site in bovine chymotrypsin A\%. A crystallographic study using peptide chloromethyl ketones as site-specific inhibitors. Biochemistry 10: 3728-3738 (1971)

174. Segal, D. M.: A kinetic investigation of the crystallographically deduced binding subsites of bovine chymotrypsin A $\gamma$. Biochemistry 11: 349 . 356 (1972)

175. Segal, D. M., G. H. Cohen, D. R. Davies, J. C. Powers, and P. E. WILcox: The stereochemistry of substrate binding to chymotrypsin A $\gamma$. Cold Spring Harbor Symp. Quant. Biol. 36: 85$90(1972)$

176. Shaw, D. C., W. H. Stein, and S. Moore: Inactivation of chymotrypsin by cyanate. J. Biol. Chem. 239: PC 671-673 (1964)

177. SHaw, E.: Site specific reagents for chymotrypsin and trypsin. Methods Enzymol. XI, 677 (1967)

178. SHAw, E. and J. Ruscica: The essentiality of histidine in the catalytic action of subtilisin. J. Biol. Chem. 243: 6312-6313 (1968)

179. Shotton, D. M., N. J. White, and H. C. WATSON: Conformational changes and inhibitor binding at the active site of elastase. Cold Spring Harbor Symp. Quant. Biol. 36: 91-105 (1972)

180. Simpson, R. T., J. F. RJORdaN, and B. L. VALLEE: Functional tyrosyl residues in the active center of bovine pancreatic carboxypeptidase
A. Biochemistry 2: 616-622 (1963)

181. SinN, L. G., O. K. Behrens and W. W. Bromer: The amino acid sequence of glucagon, IV. The hydrolysis of glucagon with subtilisin. J. Amer. Chem. Soc. 79: 2805-2807 (1957)

182. Smith, E. L., R. J. Delange, W. H. Evans, M. LANDON, and F. S. MARKIAND: Subtilisin Carlsberg. V. The complete sequence; comparison with subtilisin BPN'; evolutionary relationships. J. Biol. Chem. 243: 2184-2191 (1968)

183. Smith, E. L., F. S. Markland, and A. N. GLAZER: Some structure-function relationships in the subtilisins. In: P. Desnuelle, $H$. Neurath, and M. Ottesen, eds., StructureFunction Relationships of Proteolytic Enzymes, pp. 160-172. Munksgård (Copenhagen) 1970.

184. Sokolovsky, M., J. F. Riordan, and B. L. VALLEE: Tetranitromethane. A reagent for the nitration of tyrosyl residues in proteins. Biochemistry 5: 3582-3589 (1966)

185. Sokolovsky, M., D. Harell, and J. F. RIORDAN: Reaction of tetranitromethane with sulfhydryl groups in proteins. Biochemistry 8 : 4740-4745 (1969)

186. Sokolovsky, M., M. Fuchs, and J. F. Riordan: Reaction of tetranitromethane with tryptophan and related compounds. FEBS Lett. 7:167-170 (1970)

187. Svendsen, I.: Studies on the carbamylation of subtilisin type Novo, Compt. Rend. Trav. Lab. Carlsberg 36: 235-246 (1967)

188. SVENDSEN, I.: Chemical derivatives of subtilisins with modified proteolytic activities. I. Nitration and iodination of subtilisin type Carlsberg and subtilisin type Novo. Compt. Rend. Trav. Lab. Carlsberg 36: 347-363 (1968)

189. SVENDSEN, 1.: The inhibitory effects of alcohols and guanidine hydrochloride on the enzymatic activity of the subtilisins. Compt. Rend. Trav. Lab. Carlsberg 38: 385-397 (1971)

190. Svendsen, I.: Identification of the 3-nitrotyrosyl residues in subtilisin type Carlsberg treated with tetranitromethane. Compt. Rend. Trav. Lab. Carlsberg 39: 375-398 (1974)

190 Svendsen, I: The hydrolysis of the three

a. clupein components by subtilisin Novo, subtilisin Carlsberg and nitrated subtilisin Carlsberg. Carlsberg Res. Commun. 41: 131-142 (1976)

191. Stark, G. R. and D. G. SMYth: The use of cyanate for the determination of $\mathrm{NH}_{2}$-terminal residues in proteins. J. Biol. Chem. 238: 214-226 (1963)

192. STARK. G. R.: Reactions of cyanate with functional groups of proteins. III. Reactions with amino and carboxyl groups. Biochemistry 4: $1030-1036$ (1965)

193. STARK, G. R.: Reactions of cyanate with functional groups of proteins. II. Formation, decomposition, and properties of N-car- 
bamylimidazole. Biochemistry 4: 588-595 (1965)

194. Stark, G. R.: Reaction of cyanate with functional groups of proteins. IV. Inertness of aliphatic hydroxyl groups. Formation of carbamyl- and acylhydantoins. Biochemistry 4: 2363-2367 (1965)

195. Stauffer, C. E. and D. Etson: The effect on subtilisin activity of oxidizing a methionine residue. J. Biol. Chem. 244. 5333-5338 (1969)

196. Stroud, R. M., L. M. Kay, and R. E. DICKERSON: The crystal and molecular structure of DIP-inhibited bovine trypsin at $2.7 \AA$ resolution. Cold Spring Harbor Symp. Quant. Biol. 36: 125-140 (1972)

197. TAZAwA, Y.: Über Pflanzenproteasen. V: Zur Specificität der krystallisierten Proteinase von Bacillus subtilis. J. Biochem. (Tokyo) 42: 295-303 (1955)

198. Thompson, R. C. and E. R. Blout: Evidence for an extended active center in elastase. Proc. Natl. Acad. Sci. U. S. 67: 1734-1740 (1970)

199. Thompson, R. C.: Use of peptide aldehydes to generate transition-state analogs of elastase. Biochemistry 12: 47-51 (1973)

200. Thompson, R. C. and E. R. Blout: Dependence of the kinetic parameters for elastase-catalyzed amide hydrolysis on the length of peptide substrates. Biochemistry 12: 57-65 (1973)

201. Thompson, R. C. and E. R. Blout: Peptide chloromethyl ketones as irreversible inhibitors of elastase. Biochemistry 12: 44-47 (1973)

202. Thompson, R. C. and E. R. Blout: Elastase-catalyzed amide hydrolysis of tri- and tetrapeptide amides. Biochemistry 12: 66-71 (1973)

203. Tsernoglou, D. and G. A. Petsko: X-ray crystallography of thrombin and subtilopeptidase-A (Carlsberg). Absträct V. LinderstrømLang Conference, 1975, Vingsted, Denmark.

204. TUPPY, H.: Úber die enzymatische specificität der bakteriellen proteinase die ovalbumin in plakalbumin verwandelt. Monatsh. Chem. 84: 996 (1953)

205. TuPPY, H.: The amino-acid sequence in oxytocin. Biochim. Biophys. Acta 11: 449-450 (1953)

206. Uemitsu, N., M. Sugiyma and H. Matsumiya: The reactivation of phenylmethanesulfonyl-subtilisin. Biochim. Biophys. Acta 258: 562-565 (1972)

207. Vallee, B. L., J. F. Riordan, J. L. Bethune, T. L. CoOmBS, D. S. AulD, and M. Sokolovsky: A model for substrate binding and kinetics of carboxypeptidase A. Biochemistry 7: 3547-3556 (1968)

208. Vallee, B. L. and J. F. Riordan: Chemical approaches to the properties of active sites of enzymes. Ann. Rev. Biochemistry 38: 733-794 (1969)

209. VINCENT, J. P., M. LAzDUnSki, and M. DeLaAgE: On the use of tetranitromethane as a nitration reagent. Eur. J. Biochem. 12: 250-257 (1970)

210. Watson, H. C., D. M. Shotton, J. M. Cox, and H. MuirheaD: Three-dimensional Fourier synthesis of tosyl-elastase at $3.5 \AA$ resolution. Nature 225: 806-811 (1970)

211. Weber, B. H. and J. Kraut: Identification of the most rapidly iodinated tyrosine in subtilisin

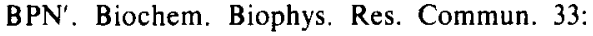
280-286 (1968)

212. Welker, N. E. and L. L. Campbell: Unrelatedness of Bacillus amyloliquefaciens and Bacillus subtilis. J. Bacteriol. 94: 1124-1130 (1967)

213. WILliams, J. and J. M. Lowe: The cross-linking of tyrosine by treatment with tetranitromethane. Biochem. J. 121: 203-209 (1971)

214. WOLFENDEN, R.: Analog approaches to the structure of the transition state in enzyme reactions. Acc. Chem. Res. 5: 10-18 (1972)

215. Vornick, I. M. and J. S. Fruton: The comparative specificity of acid proteinases. Proc. Natl. Acad. Sci. U. S. 68:257-259 (1971)

216. Wright, C. S., R. A. Alden, and J. Kraut: Structure of subtilisin BPN' $^{\prime}$ at $2.5 \dot{A}$ resolution. Nature 221: 235-242 (1969)

217. WRIGHT, C. S.: Comparison of the active stereochemistry and substrate conformation in a-chymotrypsin and subtilisin BPN'. J. Mol. Biol. 67: 151-163 (1972)

218. Wright, C. S., R. A. Alden, and J. Kraut: Crystal structure of a subtilisin $\mathrm{BPN}^{\prime}$ complex with N-benzoyl-L-arginine. J. Mol. Biol. 66:283289 (1972)

219. Yамамото, T. and N. IzumiYa: Action of trypsin on synthetic substrates. III. Hydrolysis of homologous peptides ranging from diglycylL-lysyl-glycin to di-glycyl-L-lysyl-tetraglycine. Arch. Biochem. Biophys. 120: 497-502 (1967)

220. Yoshida, N., T. Yamamoto, and N. Izumiya: Action of chymotrypsin on synthetic substrates. VII. Hydrolysis of oligopeptides containing glycine and tyrosine. Arch. Biochem. Biophys. 123: 166-171 (1968)

221. Young, J. D., E. Benjamini, M. Shimizu, and C. Y. LEUNG: Immunochemical studies on the tobacco mosaic virus protein. III. The degradation of an immunologically active tryptic peptide of tobacco mosaic virus protein and the reactivity of the degradation products with antibodies to the whole protein. Biochemistry 5 : 1481-1488 (1966) 